\title{
ON SIGNED BRANCHING MARKOV PROCESSES WITH AGE
}

\section{TUNEKITI SIRAO}

To Professor Kiyoshi Noshiro on the occasion of his 60th birthday

§ 1. Introduction. Many authors have considered branching Markov processes for the probabilistic treatment of semi-linear equations. Recently J.E. Moyal [11], [12] gave a formulation for a wide class of branching processes. A similar idea was used in A.V. Skorohod [18] and N. Ikeda-M. Nagasawa-S. Watanabe [4]-[7]. Applying their method, we shall consider in this paper the following problems $(A)$ and $(B)$.

(A): Let $E$ be a compact Hausdorff space with the second axiom of countability and assume the following are given: (1) $H_{t}$ : a strongly continuous semi-group on $\boldsymbol{C}(E)=\{f$; continuous function on $E\}$, (2) $\mathscr{G}$ : the infinitesimal operator of $H_{t}$, (3) $k(x), q_{n}(x), n=0,1,2, \cdots$, are continuous functions on $E$ such that $k(x)=\sum_{n=0}^{\infty} q_{n}(x)$ and $\sum_{n=0}^{\infty}\left|q_{n}(x)\right|<\infty$. How can we interprete probabilistically the following equation?

$$
\frac{\partial u(t, x)}{\partial t}=\mathscr{G} u(t, x)+k(x) F(x ; u(t, x)), \quad x \in E, t \geqq 0,
$$

where

$$
F(x ; \xi)=\frac{1}{k(x)} \sum_{n=0}^{\infty} q_{n}(x) \xi^{n}, \quad x \in E, \quad \xi \in R^{1} .
$$

(B): How can we interprete probabilistcally the following equation?

$$
\left.\frac{\partial u(t, x)}{\partial t}=\frac{1}{2} \Delta u(t, x)+G(u(t, x)), \quad x \in R^{d}, t>0,1\right)
$$

where $\Delta$ denotes the Laplacian in $x$ and $G(\xi)$ satisfies

$$
G(0)=G(1)=0, G(\xi)>0 \text { and } G^{\prime}(0)>G^{\prime}(\xi), \quad 0<\xi<1 .
$$

Received April 17, 1967.

1) $R^{d}$ denotes the $d$-dimensional Euclidian space. 
The equation (1.3) for more general $G$ was discussed by A. KolmogoroffI. Petrovsky-N. Piscounoff [9].

We first consider the problem $(A)$. Among others, Ikeda-NagasawaWatanabe [4]-[7] have shown that (1.1) can be interpreted probabilistically by means of branching Markov processes when the $q_{n}(x)$ are non-negative, $q_{1}(x)=0$ and

$$
F(x ; \xi)=\frac{1}{k(x)}\left\{\sum_{n \neq 1} q_{n}(x) \xi^{n}-\xi\right\},{ }^{2)} \quad x \in E, \quad \xi \in R^{1} .
$$

Hence, problem $(A)$ becomes a question of eliminating the restrictions concerning positivity of $q_{n}(x), q_{1}(x)=0$ and the term $-\xi$ in the right hand side of (1.5).

Let us next consider the following special case of (1. 1): (1) $E=R^{d} \cup\{\infty\}$ be the spaje obtained by the one-point compactification of $R^{d}$, (2) $q_{0}=q_{1}=0$ and the other $q_{n b}^{\prime} s$ are non-negative constants, (3) $\sum_{n=2}^{\infty} q_{n}=1$, (4) $\mathscr{G}=\frac{1}{2} \Delta$ and

$$
F(\xi)=\sum_{n \neq 1} q_{n} \xi^{n}-\xi
$$

Then (1. 1) becomes a special case considered by Ikeda-Nagasawa-Watanabe [6], and is written as follows:

$$
\left.\frac{\partial u(t, x)}{\partial t}=\frac{1}{2} \Delta u(t, x)+F(u(t, x)), \quad x \in R^{d}, t \geqq 0 .^{3}\right)
$$

where $F(0)=F(1)=0$. If we put $u(t, x)=1-v(t, x)$ and $G(\xi)=-F(1-\xi)$, then the above equation turns out to be the following equation

$$
\frac{\partial v(t, x)}{\partial t}=\frac{1}{2} \Delta v(t, x)+G(v(t, x)), \quad x \in R^{d}, t \geqq 0,
$$

where $G$ satisfies (1.4). This means that problem $(B)$ can be solved by means of a branching Markov process in the special case stated above.

Now, we shall sketch here the contents of $\S \S 2-8$. In $\S 2$, we shall give the notations which are used in the later discussions and give also the definitions of a branching Markov process with age and a signed branching

2) We can not regard here $q_{1}(x)=-1$ because $k(x)=\sum_{n \neq 1} q_{n}(x)$.

3) The one-point compactification of $R^{d}$ was used to apply the general theory and hence we omitted the point $\infty$ because we are interested in the equation whose variable domain is $R^{d}$. 
Markov process with age after introducing extenued state spaces $\hat{\boldsymbol{S}}$ and $\tilde{\boldsymbol{S}}$. In $\S 3$, we shall consider a branching Markov process with age $Y_{t}$ on $\hat{\boldsymbol{S}}$ satisfying Condition 1 stated there. Then, for a given system $\left\{q_{n}(x) ; n=0,2,3, \cdots\right\}$ of non-negative functions, $k(x)=\sum_{n \neq 1} q_{n}(x)$ and $F(x ; \xi)$ defined by (1.2) where $q_{1}(x)=0$, we can discuss an integral equation which corresponds to the one called "S-equation" in [7]. Under certain conditions, the integral equation can be transformed into the equation of type (1. 1). In this case, $\left.u(t, x)=T_{t} \widehat{f \cdot 2}(x, 0)^{1}\right)$ is a solution of (1. 1) with $u(0, x)=f(x)$ if $u(t, x)$ is finite. This shows that we can eliminate the term $-\xi$ in the right hand side of (1.4) by introducing of the notion of age. Moreover, for $G(\xi)=\xi^{n}$, the notion of branching Markov processes with age will serve to answer the question as to the existence of a non-trivial solution of (1.3) which does not blow up in $[0, \infty)$. (See $\S 6$ and $M$. Nagasawa-T. Sirao [14].)

In $\S 4$, we shall consider a signed branching Markov process with age $Z_{t}$ on $\tilde{\boldsymbol{S}}$ satisfying Condition 2 which is essentially identical to Condition 1 except for the difference of branching (splitting) law caused by the difference of the state spaces $\hat{\boldsymbol{S}}$ and $\tilde{\mathbf{S}}$. After making the similar considerations as in $\S 3$, we can interpret (1. 1) probabilistically. That is to say $u(t, x)=$ $\left.U_{t} \widetilde{f \cdot 2}(x, 0,0)^{5}\right)$ is a solution of (1.1) with $u(0, x)=f(x)$ if $u(t, x)$ is finite. This means that we can solve the problem $(A)$ by means of signed branching Markov processes with age. (The existence of such (signed) branching Markov processes with age discussed in $\S 3-4$ will be shown in $\S$ 7-8.)

In $\S 5$, we shall give a sufficient condition called Condition 3 in this paper which includes Condition 2 and makes a given Markov process $Z_{t}$ become a signed branching Markov process with age on $\widetilde{\boldsymbol{S}}$. This part of the present paper, Ikeda-Nagasawa-Watanabe [7] and Nagasawa [13] overlap in some respects, because the proof of Theorem 5.1 is essentially the same as one given in [7].

In $\S 6$, we shall consider a Markov process $Z_{t}$ satisfying Condition 3 whose existence is shown in $\S \S 7-8$. According to the discussions in $\S 5, Z_{t}$ is a signed branching Markov process with age. Let $f$ be a positive con-

4) $T_{t}$ denotes the semi-group induced by $Y_{t}, \widehat{f \cdot 2}$ is a function of special type defined by (2. 1) and $(x, 0) \in \hat{\boldsymbol{S}}$.

5) $U_{t}$ denotes the semi-group induced by $Z_{t}, \widetilde{f \cdot 2}$ is a function of special type defined by (2. 2$)$ and $(x, 0,0) \in \tilde{\boldsymbol{S}}$. 
tinuous function on $R^{d}$ with $\|f\|=\sup \left\{|f(x)| ; x \in R^{d}\right\}<1$ and $\alpha \in R^{1}$. When we consider $u(\alpha ; t, x)=U_{t} \alpha f \cdot 2(x, 0,0)$, where $U_{t}$ denotes the semigroup induced by $Z_{t}$ and $x \in R^{d}, u(\alpha ; t, x)$ can be expressed in the power series of $\alpha$ if $u(\alpha ; t, x)$ is finite. But the solution of (1.3) with initial value $\alpha f$, in general, can not be expressed in the power series of $\alpha$. Accordingly, if $G(\xi)$ is not an analytic function of $\xi$, then we can not obtain the solution of (1.5) with initial value $f$ directly by means of signed branching Markov processes with age as in the case of analytic $G$. However, if $G$ is continuously differentiable on $[0,1]$ and satisfies the condition (1.4), then we can express the solution $u(t, x)$ of (1.3) with initial value $f$ as the uniform limit of $u_{n}(t, x)$ in the wide sense where $u_{n}(t, x)$ is of the type considered in $\S 4$, i.e. there exists a sequence of signed branching Markov processes with age $Z_{t}^{(n)}$ on $\widetilde{\boldsymbol{S}}$ and corresponding semi-groups $U_{t}^{(n)}$ such that

$$
u(t, x)=\lim _{n \rightarrow \infty} u_{n}(t, x), \quad x \in R^{d},
$$

where

$$
u_{n}(t, x)=U_{t}^{(n)} \widetilde{f \cdot 2}(x, 0,0), \quad x \in R^{d}, t \geqq 0 .
$$

In $\S 7$, we shall construct a certain Markov process $Y_{t}$ which will be used in $\S 8$ in the constructions of branching Markov processes with age and signed branching Markov processes with age. We can regard this Markov process $Y_{t}$ as corresponding to the creation of mass in the following sense. Let $k(x)$ be a bounded continuous function on $E$ and consider the equation

$$
\frac{\partial u(t, x)}{\partial t}=\mathscr{C} u(t, x)+k(x) u(t, x), \quad x \in E, t \geqq 0,
$$

where $\mathscr{G}$ is a infinitesimal operator of a semi-group $H_{t}$ corresponding to a Markov process $X_{t}$ on $E$. If $k(x)$ is non-positive, we can treat (1.6) by killing $X_{t}$. So we may consider (1.6) as the equation corresponding to the killing when $k(x)$ is non-positive. On the other hand, we may consider (1. 6) as the equation corresponding to the creation of mass when $k(x)$ is non-negative. In the theory of Markov processes, there are, as far as I know, two methods of interpreting (1.6) when $k(x)$ is non-negative. One of them has been indicated by G.A. Hunt [3]. The other method is based on the theory of a branching Markov process, where (1.6) appears as the mean number of particles. (cf. K.Ito-H.P.McKean [8] and IkedaNagasawa-Watanabe [6] or [7]. ) Our method of describing the creation of 
mass uses age as an auxiliary variable. Let $N$ be all the non-negative integers. We shall construct a strong Markov process $\left[X_{t}, N_{t}\right]$ on the state space $E \times N$ and consider the corresponding semi-group $V_{t}$. Then, for a given bounded continuous function $f$ on $E, u(t, x)=V_{t} \widehat{f \cdot 2}(x, 0)$ is the solution of (1.6) with the initial value $f$.

In $\S 8$, we shall construct a Markov process $Z_{t}$ satisfying Condition 3. Then, by the discussions in $\S 5, Z_{t}$ is a signed branching Markov process and the existence of the processes in $\$ \S 3-4$ is proved. We here note that the method of J.E. Moyal [10] will play an essential role in the construction of the processes dealt with in $\S 8-8$.

The author wishes to express his hearty thanks to Professors N. Ikeda, M. Nagasawa and S. Watanabe who sent him preprints of their papers [4]-[7] and gave him various advice. The author also expresses his deep gratitude to Professor K. Ito who gave him valuable advice, too.

§2. Notations and Definitions. A branching process is one of the typical mathematical models used to describe the growth of the number of particles of a population in which each particle either produces new particles of the same character or dies out, and there is no interference among them. In order to describe the state of $n$ particles, it seems to be natural to use the $n$-fold symmetric direct product space of the state space of each particle. Following [4], we here introduce some notations along this line.

Let $E$ be a compact Hausdorff space satisfying the second axiom of countability. We denote the $n$-fold product space of $E$ with itself by $E^{(n)}$ and say that $\left(x_{1}^{\prime}, x_{2}^{\prime}, \cdots, x_{n}^{\prime}\right) \in E^{(n)}$ is equivalent to $\left(x_{1}, x_{2}, \cdots, x_{n}\right) \in E^{(n)}$ if and only if $\left(x_{1}^{\prime}, x_{2}^{\prime}, \cdots, x_{n}^{\prime}\right)$ is obtainable from a permutation of $\left(x_{1}, x_{2}, \cdots, x_{n}\right)$. The $E^{n}$ is defined as the quotient space of $E^{(n)}$ by the above equivalence relation. By the quotient topology, $E^{n}$ is compact. A point $\boldsymbol{x}$ in $E^{n}$ is also denoted by $\left[x_{1}, x_{2}, \cdots, x_{n}\right]$ as a collection of $n$-points $x_{i} \in E$ disregarding order. $E^{0}$ is considered as the set of the single point $\partial$, where $\partial$ denotes an extra point.

Let $N=\{0,1,2, \cdots\}$ and $N^{(n)}$ be the $n$-fold product space of $N$ with itself. A point $\left(p_{1}^{\prime}, p_{2}^{\prime}, \cdots, p_{1 b}^{\prime}\right)$ of $N^{(n)}$ is said to be equivalent to $\left(p_{1}, p_{2}, \cdots, p_{n}\right) \in N^{(n)}$ if $\sum_{i=1}^{n} p_{i}^{\prime}=\sum_{i=1}^{n} p_{i} \cdot{ }^{6)}$ The quotient space of $N^{(n)}$ by the

6) In the future discussions, $\sum_{i=1}^{n} p_{i}$ is essential in the role of $\left(p_{1}, p_{2}, \cdots, p_{n}\right)$ and hence we used here this equivalence relation. 
above equivalence relation is denoted by $N^{n}$. A point $\boldsymbol{p}$ in $N^{n}, n \geqq 1$, is a collection of equivalent points in $N^{(n)}$ and is denoted by $\left[p_{1}, p_{2}, \cdots, p_{n}\right]$ if it contains $\left(p_{1}, p_{2}, \cdots, p_{n}\right) \in N^{(n)} .|\boldsymbol{p}|$ denotes $\sum_{i=1}^{n} p_{i}$ for $\boldsymbol{p}=\left[p_{1}, p_{2}, \cdots, p_{n}\right]$. Let $S=E \times N$ be the topological sum of $E \times\{p\}, p \in N$. Then $S$ is a locally compact Hausdorff space satisfying the second axiom of countability. $S^{(n)}$ is defined as the $n$-fold product space of $S$ with itself and $\left(\left(x_{1}^{\prime}, p_{1}^{\prime}\right)\right.$, $\left.\left(x_{2}^{\prime}, p_{2}^{\prime}\right), \cdots,\left(x_{n}^{\prime}, p_{n}^{\prime}\right)\right)$ is said to be equivalent to $\left(\left(x_{1}, p_{1}\right),\left(x_{2}, p_{2}\right), \cdots,\left(x_{n}, p_{n}\right)\right)$ if $\left[x_{1}^{\prime}, x_{2}^{\prime}, \cdots, x_{n}^{\prime}\right]$ is identical to $\left[x_{1}, x_{2}, \cdots, x_{n}\right]$ as a point of $E^{n}$ and if $\left[p_{1}^{\prime}, p_{2}^{\prime}, \cdots, p_{n}^{\prime}\right]$ is identical to $\left[p_{1}, p_{2}, \cdots, p_{n}\right]$ as a point of $N^{n}$. $S^{n}$ is defined as the quotient space by the above equivalence relation. Then $S^{n}$ is locally compact with respect to the quotient topology. A point $z$ in $S^{n}$ is denoted by $\left[\left[x_{1}, x_{2}, \cdots, x_{n}\right],\left[p_{1}, p_{2}, \cdots, p_{n}\right]\right]$ or, for short, $[\boldsymbol{x}, \boldsymbol{p}]$ when $\boldsymbol{x}=\left[x_{1}, x_{2}, \cdots, x_{n}\right] \in E^{n}$ and $\boldsymbol{p}=\left[p_{1}, p_{2}, \cdots, p_{n}\right] \in N^{n}$.

Let us consider the topological sum $\bigcup_{n=0}^{\infty} S^{n}$ where $S^{0}$ denotes $\{\partial\} \times N, \partial$ being an extra point. This topological sum is denoted by $\boldsymbol{S}$. Then $\boldsymbol{S}$ is a locally compact and non-compact Hausdorff space satisfying the second axiom of countability. If we consider the mapping $g$ from $S$ to $\left(\bigcup_{n=0}^{\infty} E^{n}\right) \times N$ defined by

$$
g([\boldsymbol{x}, \boldsymbol{p}])=[\boldsymbol{x},|\boldsymbol{p}|],
$$

then $S$ is isomorphic to $\left(\bigcup_{n=0}^{\infty} E^{n}\right) \times N$, where $\bigcup_{n=0}^{\infty} E^{n}$ denotes the topological sum of $E^{n} . \quad \hat{S}=S \cup\{\Delta\}$ is defined as the space obtained by the one-point compactification of $\boldsymbol{S}$. When $A$ and $B$ are subsets of $E$ and $E^{n}$ respectively, the sets $A \times\{p\}$ and $B \times\{\boldsymbol{p}\}$ are denoted by $[A, p]$ and $[B, \boldsymbol{p}]$ respectively.

Let $J$ be the set $\{0,1,2,3\}$ and $\tilde{\boldsymbol{S}}$ be the topological sum of $\boldsymbol{S} \times\{j\}$, $j \in J$. A point in $\tilde{\boldsymbol{S}}$ is denoted by $[\boldsymbol{x}, \boldsymbol{p}, j],[\boldsymbol{x}, \boldsymbol{p}] \in \boldsymbol{S}$, but $\{\Delta\} \times J$ is considered as one point and is denoted simply by $\Delta$. Then we may consider $\widetilde{\boldsymbol{S}}$ is the space obtained by the one-point compactification of $\boldsymbol{S} \times J$. For a subset $[B, \boldsymbol{p}]$ of $\hat{\boldsymbol{S}}$, the set $[B, \boldsymbol{p}] \times\{j\}$ is denoted by $[B, \boldsymbol{p}, j]$.

Now let $\mathscr{X}$ be a compact or locally compact Hausdorff space. We shall introduce the following function spaces which are supposed to be real.

$C(\mathscr{X})=$ the set of all bounded continuous functions on $\mathscr{X}$, $\boldsymbol{B}(\mathscr{X})=$ the set of all bounded Borel measurable functions on $\mathscr{X}$, 


$$
\begin{aligned}
& \boldsymbol{C}^{*}(\mathscr{X})=\left\{f ; f \in \boldsymbol{C}(\mathscr{X}) \text { and }\|f\|=\sup _{x \in \mathscr{X}}|f(x)|<1\right\}, \\
& \boldsymbol{B}^{*}(\mathscr{X})=\{f ; f \in \boldsymbol{B}(\mathscr{X}) \text { and }\|f\|<1\} .
\end{aligned}
$$

When $\mathscr{X}$ is a locally compact Hausdorff space, let $X=\mathscr{X} \cup\{\infty\}$ be the space obtained by the one-point compactification of $\mathscr{X}$ and set

$$
\begin{aligned}
& \boldsymbol{C}_{0}(\mathscr{X})=\left\{f ; f \in \boldsymbol{C}(X), \lim _{x \rightarrow \infty} f(x)=0\right\}, \\
& \boldsymbol{C}_{0}^{*}(\mathscr{X})=\left\{f ; f \in \boldsymbol{C}_{0}(\mathscr{X}),\|f\|<1\right\} .
\end{aligned}
$$

$\boldsymbol{C}_{0}(\mathscr{X})$ and $\boldsymbol{C}_{0}^{*}(\mathscr{X})$ are denoted by $\boldsymbol{C}_{0}(X)$ and $\boldsymbol{C}_{0}^{*}(X)$ occasionally. The subclass of each function space introduced above formed of all non-negative elements is denoted by "+", e.g. $\boldsymbol{C}(\mathscr{X})^{+}, \boldsymbol{B}(\mathscr{X})^{+}, \cdots$, etc. We shall denote by " - " the closure with respect to the norm \| \|, so

$$
\begin{aligned}
& \overline{\boldsymbol{C}}^{*}(\mathscr{X})=\{f ; f \in \boldsymbol{C}(\mathscr{X}) \text { and }\|f\| \leqq 1\}, \\
& \overline{\boldsymbol{B}}^{*}(\mathscr{X})=\{f ; f \in \boldsymbol{B}(\mathscr{X}) \text { and }\|f\| \leqq 1\},
\end{aligned}
$$

and so on.

The set of all Borel subsets of $\mathscr{X}$ is denoted by $\mathscr{B}(\mathscr{X})$.

Now we shall define several operations on functions which will play an important role in the future discussions. First of all let us define a mapping from $\boldsymbol{B}(E)$ into the space of all measurable functions on $\hat{\boldsymbol{S}}$ by

$$
\widehat{f \cdot \lambda}(\boldsymbol{z})= \begin{cases}\lambda^{p}, & \text { if } \boldsymbol{z}=[\partial, p] \in S^{0} \\ \lambda^{|p|} \prod_{i=1}^{n} f\left(x_{i}\right), & \text { if } \boldsymbol{z}=[\boldsymbol{x}, \boldsymbol{p}] \in S^{n} \text { and } \boldsymbol{x}=\left[x_{1}, x_{2}, \cdots, x_{n}\right], \\ 0, & \text { if } \boldsymbol{z}=\Delta,\end{cases}
$$

where $\lambda \geqq 0$. If $f \in \boldsymbol{C}^{*}(E)$ and $0 \leqq \lambda<1$, then $\widehat{f \cdot \lambda} \in \boldsymbol{C}_{0}^{*}(\hat{\boldsymbol{S}})$, while $\widehat{f \cdot \lambda}$ is unbounded for $\lambda>1$.

Next we shall define a mapping $\sim$ from $\boldsymbol{B}(E)$ into the space of all measurable functions on $\tilde{\boldsymbol{S}}$ by

$$
\widetilde{f \cdot \lambda}([\boldsymbol{x}, \boldsymbol{p}, j])=(-1)^{\left[\frac{j}{2}\right]} \widehat{f \cdot \lambda}([\boldsymbol{x}, \boldsymbol{p}]), \quad[\boldsymbol{x}, \boldsymbol{p}, j] \in \widetilde{\boldsymbol{S}},
$$

where $\lambda \geqq 0$ and [ ] denotes Gauss' symbol.

For any function $g$ on $\hat{S}$, we define a function $\left.g\right|_{E}$ on $E$ by

$$
\left.g\right|_{E}(x)=g([x, 0]), \quad x \in E .
$$


We define also $\left.h\right|_{E}$ for any function on $\tilde{\boldsymbol{S}}$ by

$$
\left.h\right|_{E}(x)=h(x, 0,0), \quad x \in E .
$$

Remark 1. Let $\left(\bigcup_{n=0}^{\infty} E^{n}\right) \cup\{\Delta\}$ be the space obtained by the one-point compactification of $\bigcup_{n=0}^{\infty} E^{n}$. Ikeda-Nagasawa-Watanabe [4]-[7], used a map-

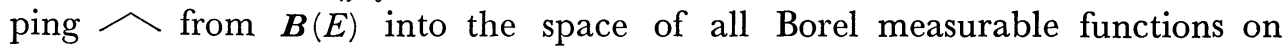
$\left(\bigcup_{n=0}^{\infty} E^{n}\right) \cup\{\Delta\}$ defined by

$$
\hat{f}(\boldsymbol{x})= \begin{cases}1, & \text { if } \boldsymbol{x}=\partial, \\ \prod_{i=1}^{n} f\left(x_{i}\right), & \text { if } \boldsymbol{x}=\left[x_{1}, x_{2}, \cdots, x_{n}\right] \in E^{n}, \\ 0, & \text { if } \boldsymbol{x}=\Delta .\end{cases}
$$

Then the linear hull of the set $\left\{\hat{f} ; f \in C^{*}(E)^{+}\right\}$is dense in $\boldsymbol{C}_{0}\left(\bigcup_{n=1}^{\infty} E^{n}\right)$ (cf. Lemma 1.4 in [7]). Accordingly, the linear hull of the set $\widehat{\{f \cdot \lambda} ; f \in C^{*}(E)^{+}$, $0 \leqq \lambda<1\}$ is dense in $\boldsymbol{C}_{0}(\hat{\boldsymbol{S}})$ because the linear hull of $\left\{\lambda ; \lambda(p)=\lambda^{p}, 0 \leq \lambda<1\right.$, $p \in N\}$ is also dense in $\boldsymbol{C}_{0}(N)$.

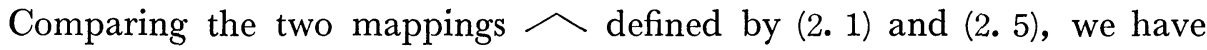

$$
\widehat{f \cdot 1}([\boldsymbol{x}, \boldsymbol{p}])=\hat{f}([\boldsymbol{x}]) \text {. }
$$

So we need not distinguish between $\widehat{f} \cdot 1$ and $\hat{f}$ if there arises no danger of confusion.

Now we shall consider a Markov process $X=\left\{X_{t}, \zeta, \mathscr{B}_{t}, P_{x} ; x \in \mathscr{X}\right\}$ on $\mathscr{X}$. Let $\mathscr{B}_{\infty}$ be the smallest $\sigma$-algebra which contains all elements of $\mathscr{B}_{t}$ for any $t \geqq 0$. A non-negative random variable $\tau$ is said to be a $\left(\mathscr{B}_{t^{-}}\right)$ Markov time if

$$
\{w ; \tau(w) \leqq t\} \in \mathscr{B}_{t},
$$

for any $t \geqq 0$. For each Markov time $\tau$, we set

$$
\mathscr{B}_{\tau}=\left\{A ; A \in \mathscr{B}_{\infty} \text { and } A \cap\{w ; \tau(w) \leqq t\} \in \mathscr{B}_{t} \text { for any } t \geqq 0\right\} \text {. }
$$

Then it is easy to see that $\mathscr{B}_{\tau}$ is a $\sigma$-algebra. A measurable Markov process $X$ is called a strong Markov process if for any Markov time $\tau$ and for any $t \geqq 0, x \in \mathscr{X}, f \in \boldsymbol{B}(\mathscr{X})$ and $A \in \mathscr{B}_{\tau}$

$$
E_{x}\left[f\left(X_{t+\tau}\right) ; A \cap\{\tau<\zeta\}\right]=E_{x}\left[E_{X_{\tau}}\left[f\left(X_{t}\right)\right] ; A \cap\{\tau<\zeta\}\right],
$$


where $E_{x}$ denotes the integral by $P_{x}$.

In this paper, with the exception of $\S \S 7-8$, we shall assume that each sample function of a Markov process is right continuous in $t$ and has its left limit at any $t>0$. We also use the same letter $\zeta$ for the terminal times of different Markov processes $X$ and $Y$ and the same letter $\mathscr{B}_{t}$ for the corresponding $\sigma$-algebras which make $X_{t}$ or $Y_{t}$ measurable if there arises no danger of confusion.

Let $Y=\left\{Y_{t}=\left[X_{t}, N_{t}\right], \zeta, \mathscr{B}_{t}, P_{[\boldsymbol{x}, \boldsymbol{p}]} ;[\boldsymbol{x}, \boldsymbol{p}] \in \hat{\boldsymbol{S}}\right\}$ be a strong Markov process on $\hat{\boldsymbol{S}}$, where $\left[X_{t}(w), N_{t}(w)\right]=[\boldsymbol{x}, \boldsymbol{p}]$ means $X_{t}(w)=\boldsymbol{x}$ and $N_{t}(w)=\boldsymbol{p}$. We shall define the functionals of $Y$ by

$$
\begin{aligned}
& \xi_{t}(w)= \begin{cases}n, & \text { if }\left[X_{t}(w), N_{t}(w)\right] \in S^{n}, \quad n \geqq 0 \\
\infty, & \text { if }\left[X_{t}(w), N_{t}(w)\right]=\Delta,\end{cases} \\
& \tau(w)=\inf \left\{t>0 ; \xi_{t}(w) \neq \xi_{0}(w) \text { or } \sup _{s \leqq t}\left|N_{s}(w)\right|=\infty\right\}^{7)},
\end{aligned}
$$

$$
\begin{aligned}
& \sigma(w)=\inf \left\{t<\tau(w) ;\left|N_{t}(w)\right| \neq\left|N_{0}(w)\right|\right\}, \\
& \tau_{0}(w)=0, \quad \tau_{1}(w)=\tau(w), \tau_{n+1}(w)=\tau_{n}(w)+\theta \tau_{n} \tau(w), \quad(n \geqq 1), \\
& \sigma_{0}(w)=0, \sigma_{1}(w)=\sigma(w) \text { and } \sigma_{n+1}(w)=\sigma_{n}(w)+\theta_{\sigma_{n}} \sigma(w), \quad(n \geqq 1),
\end{aligned}
$$

where $\theta$ denotes the shift operator (cf. E.B. Dynkin [1]).

Further let $Z=\left\{Z_{t}=\left[X_{t}, N_{t}, J_{t}\right], \zeta, \mathscr{B}_{t}, P_{[\boldsymbol{x}, \boldsymbol{p}, j]} ;[\boldsymbol{x}, \boldsymbol{p}, j] \in \widetilde{\boldsymbol{S}}\right\}$ be a strong Markov process on $\tilde{\boldsymbol{S}}$, where $\left[X_{t}(w), N_{t}(w), J_{t}(w)\right]=[\boldsymbol{x}, \boldsymbol{p}, j]$ means $X_{t}(w)=\boldsymbol{x}$, $N_{t}(w)=\boldsymbol{p}$ and $J_{t}(w)=j$. Then we define the functionals of $Z$ by

$$
\begin{aligned}
& \eta(w)=\inf \left\{t>0 ; J_{t}(w) \neq J_{0}(w) \text { or } \sup _{s \leqq t}\left|N_{s}\right|(w) \mid=\infty\right\}, \\
& \sigma(w)=\inf \left\{t<\eta(w) ;\left|N_{t}(w)\right| \neq\left|N_{0}(w)\right|\right\}, \\
& \eta_{0}(w)=0, \quad \eta_{1}(w)=\eta(w), \eta_{n+1}(w)=\eta_{n}(w)+\theta_{\eta_{n}} \eta(w), \quad(n \geqq 1), \\
& \left.\sigma_{0}(w)=0, \sigma_{1}(w)=\sigma(w) \text { and } \sigma_{n+1}(w)=\sigma_{n}(w)+\theta_{\sigma_{n}} \sigma(w),(n \geqq 1) .8\right)
\end{aligned}
$$

Evidently $\tau_{n}, \eta_{n}$ and $\sigma_{n}$ are Markov times.

7) In this paper, we regard that inf $\phi=\infty$ where $\phi$ denotes the empty set.

8) Two functional $\sigma$ 's defined for $Y$ and $Z$ are denoted by the same letter because the definitions are identical except for the conditions $t<\tau$ and $t<\eta$, and this notation is convenient for the later use. Also, $Y$ and $Z$ are different Markov processes on the different state space $\hat{\boldsymbol{S}}$ and $\tilde{\boldsymbol{S}}$, and accordingly there arises no danger of confusion when we use the same letter $\sigma$. 
Now we shall give here the definitions of a branching Markov process with age on $\hat{\boldsymbol{S}}$ and a signed branching Markov process with age on $\tilde{\boldsymbol{S}}$.

Definition 2. 1. A strong Markov process $Y=\left\{Y_{t}=\left[X_{t}, N_{t}\right], \zeta, \mathscr{B}_{t}\right.$, $\left.P_{[\boldsymbol{x}, \boldsymbol{p}]} ;[\boldsymbol{x}, \boldsymbol{p}] \in \boldsymbol{S}\right\}$ is said to be a branching Markov process with age, if the semigroup $\left\{T_{t} ; t \geqq 0\right\}$ on $\boldsymbol{B}(\hat{\boldsymbol{S}})$ induced by $Y$ satisfies

$$
T_{t} \widehat{f \cdot \lambda}=\widehat{\left.\left(T_{t} \widehat{f \cdot \lambda}\right)\right|_{E} \cdot \lambda}, \quad f \in C^{*}(E),
$$

where $t \geqq 0$ and $0 \leqq \lambda<1$.

Definition 2. 2. A strong Markov process $Z=\left\{Z_{t}=\left[X_{t}, N_{t}, J_{t}\right], \zeta, \mathscr{B}_{t}\right.$, $\left.P_{[\boldsymbol{x}, \boldsymbol{p}, j]} ;[\boldsymbol{x}, \boldsymbol{p}, j] \in \widetilde{\boldsymbol{S}}\right\}$ is said to be a signed branching Markov process with age, if the semi-group $\left\{U_{t} ; t \geqq 0\right\}$ on $\boldsymbol{B}(\widetilde{\boldsymbol{S}})$ induced by $Z$ satisfies

$$
U_{t} \widetilde{f \cdot \lambda}=\widetilde{\left.\left(U_{t} \widetilde{f \cdot \lambda}\right)\right|_{E} \cdot \lambda,}, f \in C^{*}(E),
$$

where $t \geqq 0$ and $0 \leqq \lambda<1$.

In both processes $Y$ and $Z,\left|N_{t}\right|$ is considered as the total age of the particles and hence $\sigma_{n}$ is called the $n$th jumping time of age $N_{t} \cdot \tau_{n}$ and $\eta_{n}$ are called the $n$th branching times of $Y$ and $Z$ respectively.

Remark 2. As was mentioned already, the linear hull of $\{\widehat{f \cdot \lambda}$; $\left.f \in \boldsymbol{C}^{*}(E), 0 \leqq \lambda<1\right\}$ is dense in $\boldsymbol{C}_{0}(\hat{\boldsymbol{S}})$. Hence the process on $\boldsymbol{S}$ is uniquely determined by the values of $T_{t} \widehat{f \cdot \lambda}$ considered in (2.8). But, unfortunately, the same unique insistence does not hold for the case of $U_{t}$ (cf. Remark 2 in $\S 4$ ).

Remark 3. When $Y_{t}=\left[X_{t}, N_{t}\right]$ is a branching Markov process with age, $[\partial, p], p \in N$, and $\Delta$ are traps because

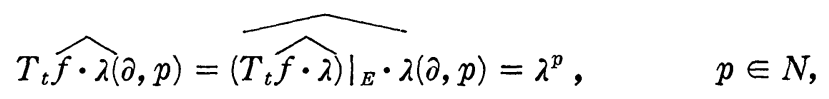

and

$$
T_{t} \widehat{f \cdot \lambda}(\Delta)=\widehat{\left.\left(T_{t} \widehat{f \cdot \lambda}\right)\right|_{E} \cdot \lambda(\Delta)}=0,
$$

for any $f \in C^{*}(E)^{+}$and $0 \leqq \lambda<1.9^{9}$

9) cf. [6], Theorem 2.1 . 
Remark 4. For any bounded continuous function $f$ on $E, \alpha f \in C^{*}(E)$ if $|\alpha|<1 /\|f\|$. So, if (2. 8) holds then we have for $f \in \boldsymbol{C}(E)-\boldsymbol{C}^{*}(E)$

$$
T_{t} \widehat{\alpha f \cdot \lambda}=\widehat{\left.\left(T_{t} \widehat{\alpha f \cdot \lambda}\right)\right|_{E}} \cdot \lambda, \quad|\alpha|<1 /\|f\| .
$$

On the other hand, both sides of the above equation can be expressed in the power series of $\alpha$. So, if we put

$$
T_{t} \widehat{\alpha f \cdot \lambda}([\boldsymbol{x}, \boldsymbol{p}])=\sum_{n=0}^{\infty} E_{[\boldsymbol{x}, \boldsymbol{p}]}\left[\widehat{\alpha f \cdot \lambda}\left(Y_{t}\right) ; Y_{t} \in S^{n}\right]=\sum_{n=0}^{\infty} a_{n}([\boldsymbol{x}, \boldsymbol{p}]) \alpha^{n}
$$

and

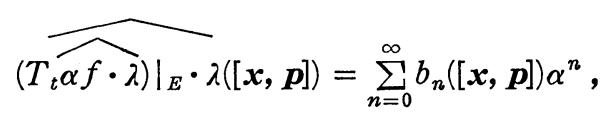

then (2. 10) shows that

$$
a_{n}([\boldsymbol{x}, \boldsymbol{p}])=b_{n}([\boldsymbol{x}, \boldsymbol{p}]), \quad n=0,1,2, \cdots
$$

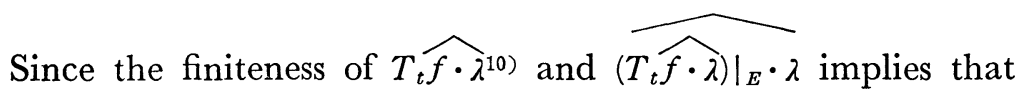

$$
\sum_{n=0}^{\infty}\left|a_{n}([x, p])\right|<\infty, \sum_{n=0}^{\infty} \mid b_{n}([x, p])<\infty
$$

and

$$
\begin{aligned}
& T_{t} \widehat{f \cdot \lambda}([\boldsymbol{x}, \boldsymbol{p}])=\sum_{n=0}^{\infty} a_{n}([\boldsymbol{x}, \boldsymbol{p}]), \\
& \widehat{\left.\left(T_{t} \widehat{f \cdot \lambda}\right)\right|_{E} \cdot \lambda([\boldsymbol{x}, \boldsymbol{p}])}=\sum_{n=0}^{\infty} b_{n}([\boldsymbol{x}, \boldsymbol{p}]),
\end{aligned}
$$

we have from (2. 11)

$$
T_{t} \widehat{f \cdot \lambda}=\widehat{\left.\left(T_{t} \widehat{f \cdot \lambda}\right)\right|_{E} \cdot \lambda}, \quad f \in C(E), 0 \leqq \lambda<1,
$$

if both sides of the above equation are finite. By the same way, we may consider that if $U_{t}$ satisfies (2.9) and both sides of the following equation are finite then we have

10) For any semi-group $T_{t}$ on $\boldsymbol{B}(\mathscr{X})$ induced by a Markov process $X_{t}$ on $\mathscr{X}$, we denote $E_{x}\left[f\left(X_{t}\right)\right]$ by $T_{t} f(x)$ in this paper even if $f$ is unbounded but $E_{x}\left[\left|f\left(X_{t}\right)\right|\right]$ is finite. $\left(E_{x}\right.$ denotes the integral by the probability measure $P_{x}$ of $X_{t}$.) 


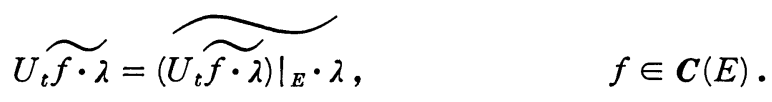

Similarly, we can see that

$$
T_{t} \widehat{f \cdot \lambda}=\widehat{\left.\left(T_{t} \widehat{f \cdot \lambda}\right)\right|_{E} \cdot \lambda}, \quad f \in \boldsymbol{C}(E), 0 \leqq \lambda,
$$

and

$$
U_{t} \widetilde{f \cdot \lambda}=\widetilde{\left.\left(U_{t} \widetilde{f \cdot \lambda}\right)\right|_{E} \cdot \lambda,}, \quad f \in \boldsymbol{C}(E), 0 \leqq \lambda,
$$

if each member of (2.12) and (2.13) is finite.

§3. Branching Markov process with age. In this section, we restrict our attention to a branching Markov process with age $Y=\left\{Y_{t}=\left[X_{t}, N_{t}\right]\right.$, $\left.\zeta, \mathscr{B}_{t}, P_{[\boldsymbol{x}, \boldsymbol{p}]} ;[\boldsymbol{x}, \boldsymbol{p}] \in \boldsymbol{S}\right\}$ satisfying the following condition (Condition 1), because it is sufficient to consider such a process for the probabilistic interpretation of equations of type (1. 1).

Let $\left\{q_{n}(x) ; n=0,2,3, \cdots\right\}$ be a given system of bounded continuous and non-negative functions on $E$, let $k(x)=\sum_{n \neq 1} q_{n}(x)$ also be a non-negative bounded continuous function on $E$, and set

$$
\begin{gathered}
\pi([x, p] ;[B, \boldsymbol{q}])=\sum_{n \neq 1} \frac{q_{n}(x)}{k(x)} \boldsymbol{\delta}_{n}([\boldsymbol{x}, \boldsymbol{p}],[B, \boldsymbol{q}]), \\
{[x, p] \in S,[B, \boldsymbol{q}] \in \mathscr{B}(\hat{\boldsymbol{S}}),}
\end{gathered}
$$

where $\delta_{n}$ is defined by

$\delta_{n}([x, p],[B, \boldsymbol{q}])= \begin{cases}1, & \text { if } \boldsymbol{x}=[x, x, \cdots, x] \in B \cap E^{n},|\boldsymbol{q}|=p, n \neq 1 \\ 0, & \text { otherwise. }\end{cases}$

Now we shall state the following

Condition 1. (i)

$$
\begin{gathered}
P_{[x, p]}\left(X_{t} \in A, N_{t}=p+q, t<\tau\right)=P_{[x, 0]}\left(X_{t} \in A, N_{t}=q, t<\tau\right), \\
{[x, p] \in S, q \in N, A \in \mathscr{B}(E) .}
\end{gathered}
$$

(ii) There exists a conservative Feller process ${ }^{11)} X^{\prime}=\left\{X_{t}^{\prime}, \mathscr{B}_{t}^{\prime}, P_{x} ; x \in E\right\}$ on $E$ such that

11) A right continuous strong Markov process on $\mathscr{X}$ is said to be a Feller process if the corresponding semi-group $T_{t}$ maps $C(\mathscr{X})$ into itself. 


$$
P_{[x, 0]}\left(X_{\tau-} \in A, \tau \in d t, \sigma_{n} \leqq t<\sigma_{n+1}\right)
$$

(3. 3)

$$
=E_{x}\left[e^{-2 \int_{o}^{t} k\left(X_{s}^{\prime}\right) d s} \frac{\left(\int_{0}^{t} k\left(X_{s}^{\prime}\right) d s\right)^{n}}{n !} k\left(X_{t}^{\prime}\right) I_{A}\left(X_{t}^{\prime}\right) d t\right]
$$

and

$$
\begin{gathered}
P_{[x, 0]}\left(X_{\sigma_{-}} \in A, \quad \sigma \in d t\right)=E_{x}\left[e^{-2 \int_{0}^{t} k\left(X_{s}^{\prime}\right) d s} k\left(X_{t}^{\prime}\right) I_{A}\left(X_{t}^{\prime}\right) d t\right], \\
{[x, 0] \in S, x \in E, \quad A \in \mathscr{B}(E),}
\end{gathered}
$$

where $E_{x}$ denotes the integral by $P_{x}$ and $I_{A}$ denotes the indicator function of $A$.

(iii) For any $\alpha>0$,

$$
\begin{aligned}
& E_{[x, p]}\left[e^{-\alpha \tau} ;\left[X_{\tau}, N_{\tau}\right] \in[B, \boldsymbol{q}]\right] \\
= & E_{[x, p]}\left[e^{-\alpha \tau} \pi\left(\left[X_{\tau-}, N_{\tau-}\right] ;[B, \boldsymbol{q}]\right)\right], \\
& {[x, p] \in S,[B, \boldsymbol{q}] \in \mathscr{B}(\boldsymbol{S}), }
\end{aligned}
$$

and

$$
\begin{aligned}
& E_{[x, 0]}\left[e^{-\alpha \sigma} ;\left[X_{\sigma}, N_{\sigma}\right] \in[A, q]\right] \\
= & E_{[x, 0]}\left[e^{-\alpha \sigma} \delta_{\left[X_{\sigma-}, N_{\sigma-+1}\right.}([A, q])\right], \\
& {[x, 0] \in S, A \in \mathscr{B}(E), q \in N, }
\end{aligned}
$$

where $E_{[x, p]}$ denotes the integral by $P_{[x, p]}, \pi$ is given in (3.1) and

$$
\delta_{[x, p]}([A, q])= \begin{cases}1, & \text { if } x \in A, p=q \\ 0, & \text { otherwise. }\end{cases}
$$

For the process $X^{\prime}$ considered in (ii), we give the following

Definition 3. 1. The process $X^{\prime}$ is called the basic Markov process of $Y$.

In the following, we consider the process $Y_{t}^{o}=\left[X_{t}^{0}, N_{t}^{0}\right]$ defined in the following way:

$$
Y_{t}^{0}(w)=\left\{\begin{array}{cl}
Y_{t}(w), & \text { if } t<\tau(w) \\
\Delta, & \text { if } t \geqq \tau(w) .
\end{array}\right.
$$

The probability measure for $Y_{t}^{0}$ is denoted by $P_{[x, p]}^{0}$ and the integral by 
$P_{[\boldsymbol{x}, \boldsymbol{p}]}^{0}$ is denoted by $E_{[\boldsymbol{x}, \boldsymbol{p}]}^{0}$. The semi-group on $\boldsymbol{B}(\hat{\boldsymbol{S}})$ induced by $Y_{t}^{0}$ is denoted by $T_{t}^{0}$. Accordingly, we have for any $g \in \boldsymbol{B}(\boldsymbol{S})$ with $g(\boldsymbol{\Delta})=0$

$$
\begin{gathered}
T_{t}^{0} g([\boldsymbol{x}, \boldsymbol{p}])=E_{[\boldsymbol{x}, \boldsymbol{p}]}^{0}\left[g\left(Y_{t}^{0}\right)\right]=E_{[\boldsymbol{x}, \boldsymbol{p}]}\left[g\left(Y_{t}\right) ; t<\tau\right], \\
{[\boldsymbol{x}, \boldsymbol{p}] \in \hat{\boldsymbol{S}} .}
\end{gathered}
$$

Definition 3. 2. When we restrict the starting points of $Y_{t}^{0}$ on $S, Y_{t}^{0}$ is called the non-branching part of $Y$.

Now we shall return to the discussion of Condition 1 . (i) of the condition states that if we consider the process $\left[X_{t}^{0}, N_{t}^{0}-p\right]$ for the nonbranching part $Y_{t}^{0}=\left[X_{t}^{0}, N_{t}^{0}\right]$ started from $[x, p] \in S$, then $\left[X_{t}^{0}, N_{t}^{0}-p\right]$ is stochastically equivalent to the one started from $[x, 0] \in S$. (ii) of the condition states the relation between the first branching time $\tau$ and the $n$th jumping time $\sigma_{n}$ of $N_{t}$. This condition holds if we consider a process such that $(a)$ if we set, for $Y_{t}=\left[X_{t}, N_{t}\right]$ starting from $[x, 0] \in S$,

$$
X_{t}^{0}=\left\{\begin{aligned}
X_{t}, & \text { if } t<\sigma(w) \wedge \tau(w)^{12)} \\
\Delta, & \text { if } t \geqq \sigma(w) \wedge \tau(w),
\end{aligned}\right.
$$

then $X_{t}^{0}$ is stochastically equivalent to the $\exp \left(-2 \int_{0}^{t} k\left(X_{s}^{\prime}\right) d s\right)$ sub-process of $X^{\prime}$ as a process on $E,(b)$ each path of $Y_{t}$ jumps from $\left[X_{\sigma(w) \wedge \tau(w)-}, 0\right]$ to either one of $\left[X_{\sigma(w) \wedge \tau(w)-}, 1\right]$ or some point in $S-S$ at the time $\sigma \wedge \tau$ with probability $1 / 2$. On the other hand (iii) states the branching law at the first branching time $\tau$ and the jumping law at the first jumping time $\sigma$ of $N_{t}$. (We shall show in $\S 8$ that there exists a branching Markov process with age on $\hat{\boldsymbol{S}}$ which satisfies Condition 1.) Moreover, if we combine (iii) with the stochastic equivalence of $\left[X_{t}^{0}, N_{t}^{0}-p\right]$ where $N_{0}^{0}=p$ and the nonbranching part where $N_{0}^{0}=0$, then the strong Markov property of $Y$ yields that for any $n \geqq 1$

$$
P_{[x, p]}\left(N_{\sigma_{n}} \neq N_{\sigma_{n 1}}+1, \sigma_{n}<\tau\right)=0
$$

or for any $n \geqq 1$ and $C \in \mathscr{B}_{\sigma_{n}}$,

$$
\begin{aligned}
& P_{[x, p]}\left(C, N_{\sigma_{n}}=N_{\sigma_{n-1}}+1, \sigma_{n}<\tau\right)=P_{[x, p]}\left(C, \sigma_{n}<\tau\right), \quad[x, p] \in S, \\
& E_{[x, p]}\left[e^{-\alpha \sigma} ;\left[X_{\sigma}, N_{\sigma}\right] \in[A, q]\right] \\
= & E_{[x, p]}\left[e^{-\alpha \sigma} \delta_{\left[X_{\sigma_{-}}, N_{\left.\sigma_{-}+1\right]}\right.}([A, q])\right], \quad \alpha>0,[x, p] \in S, A \in \mathscr{B}(E),
\end{aligned}
$$

12) $\sigma(w) \wedge \tau(w)$ denotes the minimum of $\sigma(w)$ and $\tau(w)$. 
and

$$
\begin{gathered}
P_{[x, p]}\left(\left[X_{\tau}, N_{\tau}\right] \in[B, \boldsymbol{p}+\boldsymbol{q}]\right)=P_{[x, 0]}\left(\left[X_{\tau}, N_{\tau}\right] \in[B, \boldsymbol{q}]\right), \\
{[x, p] \in S, B \in \mathscr{B}\left(\bigcup_{n=0}^{\infty} E^{n}\right),}
\end{gathered}
$$

where $\boldsymbol{p}+\boldsymbol{q}$ denotes $\left[p+q_{1}, q_{2}, \cdots, q_{n}\right]$ for $\boldsymbol{q}=\left[q_{1}, q_{2}, \cdots, q_{n}\right]$.

We have also from (ii) and the stochastic equivalence of $\left[X_{t}^{0}, N_{t}^{0}-N_{0}^{0}\right]$ stated above

$$
P_{[x, p]}\left(X_{\tau-} \in A, \tau \in d t, \sigma_{n} \leqq t<\sigma_{n+1}\right)
$$

$$
=E_{x}\left[e^{-2 \int_{0}^{t} k\left(X_{s}^{\prime}\right) d s} \frac{\left(\int_{0}^{t} k\left(X_{s}^{\prime}\right) d s\right)^{n}}{n !} k\left(X_{s}^{\prime}\right) I_{A}\left(X_{t}^{\prime}\right) d t\right]
$$

and

$$
P_{[x, p]}\left(X_{\sigma_{n+1}} \in A, \sigma_{n+1} \in d t\right)
$$

$$
\begin{gathered}
=E_{x}\left[e^{-2 \int_{0}^{t} k\left(X_{s}^{\prime}\right) d s} \frac{\left(\int_{0}^{t} k\left(X_{s}^{\prime}\right) d s\right)^{n}}{n !} k\left(X_{t}^{\prime}\right) I_{A}\left(X_{t}^{\prime}\right) d t\right], \\
n \geqq 0,[x, p] \in S, A \in \mathscr{B}(E) .
\end{gathered}
$$

Now we shall consider a family of measures $K([x, 0] ; \cdot, \cdot)$ on $\mathscr{B}([0, \infty) \times S)$ defined as follows: let $Y_{t}^{0}$ be the non-branching part of $Y_{t}$ and set

$$
\begin{gathered}
K([x, 0] ; d t,[A, p])=P_{[x, 0]}^{0}\left(\tau \in d t, Y_{\tau^{-}}^{0} \in[A, p]\right), \\
{[x, 0] \in S, A \in \mathscr{B}(E), p \in N .}
\end{gathered}
$$

Evidently $K([x, 0] ; \cdot, \cdot)$ is a measure on $\mathscr{B}([0, \infty) \times S)$. Moreover, by (3. 7) and (3. 10), $K([x, 0] ; \cdot, \cdot)$ can be expressed in the following form:

$$
\begin{gathered}
K([x, 0] ; d t,[A, p])=E_{x}\left[e^{-2 \int_{0}^{t} k\left(X_{s}^{\prime}\right) d s} \frac{\left(\int_{0}^{t} k\left(X_{s}^{\prime}\right) d s\right)^{p}}{p !} k\left(X_{t}^{\prime}\right) I_{A}\left(X_{t}^{\prime}\right) d t\right], \\
p \in N, A \in \mathscr{B}(E) .
\end{gathered}
$$

Further let $T_{t}^{0}$ be the semi-group on $\boldsymbol{B}(S)$ induced by $Y_{t}^{0}$ and $F$ be a function defined by

$$
F(x ; \xi)=\sum_{n \neq 1} \frac{q_{n}(x)}{k(x)} \xi^{n}, \quad x \in E, \quad \xi \in R^{1},
$$

where $q_{n}(x)$ and $k(x)$ are functions considered in Condition 1 (or in (3.1)). 
For a given system $\left(T_{t}^{0}, K, F\right)$, consider the following equation:

$$
\begin{aligned}
& u(t, x)=T_{t}^{\jmath} \widehat{f \cdot \lambda([x, 0])}+\int_{0}^{t} \int_{s} K([x, 0] ; d s,[d y, p]) \lambda^{p} F(y ; u(t-s, y)), \\
& f \in \boldsymbol{C}(E), 0 \leqq \lambda, 0 \leqq t \leqq T, x \in E,
\end{aligned}
$$

where $T$ is a positive constant.

Then we have

Lemma 3. 1. Let $T_{t}$ be the semi-group on $\boldsymbol{B}(\hat{\boldsymbol{S}})$ induced by a branching Markov process with age $Y_{t}$ on $S$ satisfying Condition 1 and let $T_{t}^{0}$ be the semigroup on $\boldsymbol{B}(\boldsymbol{S})$ induced by the non-branching part $Y_{t}^{0}$ of $Y_{t}$. Let also $f$ be a bounded continuous function on $E$. If $u(t, x)=\left.\left(T_{t} \widehat{f} \cdot \lambda\right)\right|_{E}(x)$ is finite for any $x \in E$ and $0 \leqq t \leqq T$, then $u(t, x)$ satisfies (3. 15).

REMark 1. With the exception of $\S 6,\left."\left(T_{t} \widehat{f \cdot \lambda}\right)\right|_{E}(x)$ is finite" means in this paper that

$$
E_{[x, 0]}\left[|\widehat{f} \cdot \lambda|\left(Y_{t}\right)\right]<\infty .
$$

(cf. Foot-note 10)). Let us set for any Borel measurable function $g$ on $\hat{S}$

$$
g_{n}([\boldsymbol{x}, \boldsymbol{p}])=\left\{\begin{array}{cl}
g([\boldsymbol{x}, \boldsymbol{p}]), & \text { if }|g([\boldsymbol{x}, \boldsymbol{p}])| \leqq n, \\
0, & \text { otherwise. }
\end{array}\right.
$$

If it holds that

$$
E_{[x, p]}\left(\left|g\left(Y_{t}\right)\right|\right)<\infty,
$$

then, by the strong Markov property of $Y_{t}$, we have for any Markov time $\sigma$

$$
\begin{aligned}
& E_{[x, p]}\left[\left|g\left(Y_{t}\right)\right| ; \sigma<t\right] \\
= & \lim _{n \rightarrow \infty} E_{[x, p]}\left[\left|g_{n}\left(Y_{t}\right)\right| ; \sigma<t\right] \\
= & \lim _{n \rightarrow \infty} E_{[x, p]}\left[T_{t-\sigma}\left|g_{n}\right|\left(Y_{\sigma}\right) ; \sigma<t\right]^{13)} \\
= & E_{[x, p]}\left[T_{t-\sigma}|g|\left(Y_{\sigma}\right) ; \sigma<t\right],
\end{aligned}
$$

Hence, if (3. 16) holds, then we have

$$
E_{[x, p]}\left[\left|g\left(Y_{t}\right)\right| ; \sigma<t\right]<\infty
$$

13) $T_{t-\sigma} g\left(Y_{\sigma}\right)$ or $E_{Y_{\sigma}}\left[g\left(Y_{t-\sigma}\right)\right]$ denote $E_{Y_{\sigma}}\left[g\left(Y_{t-s}\right)\right]$ at $s=\sigma$. 
and

$$
E_{[x, p]}\left[g\left(Y_{t}\right) ; \sigma<t\right]=E_{[x, p]}\left[E_{\left[X_{\sigma}, N_{\sigma}\right]}\left[g\left(Y_{t-\sigma}\right)\right] ; \sigma<t\right] .
$$

Proof of Lemma 3. 1. By the strong Markov property of $Y_{t}$ and (3. 17), it holds that

$$
\begin{gathered}
u(t, x)=E_{[x, 0]}\left[\widehat{f \cdot \lambda}\left(Y_{t}\right) ; t<\tau\right]+E_{[x, 0]}\left[\widehat{f \cdot \lambda}\left(Y_{t}\right) ; \tau \leqq t\right] \\
=T_{t}^{0} \widehat{f \cdot \lambda}([x, 0])+E_{[x, 0]}\left[E_{\left[X_{\tau}, N_{\tau}\right]}\left[\widehat{f \cdot \lambda}\left(Y_{t-\tau}\right)\right] ; \tau \leqq t\right] .
\end{gathered}
$$

If we apply the branching property $(2.12)$ to the second term of the right hand side of (3. 18), then we can see

$$
\begin{aligned}
& E_{\left[X_{\tau}, N_{\tau}\right]}\left[\widehat{f \cdot \lambda}\left(Y_{t-\tau}\right)\right]=T_{t-\tau} \widehat{f \cdot \lambda}\left(\left[X_{\tau}, N_{\tau}\right]\right)
\end{aligned}
$$

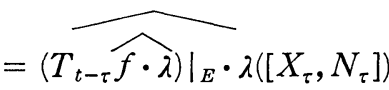

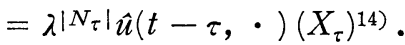

Combining (3. 1), (3. 5), (3. 12) with the above equation, we have

$$
\begin{aligned}
& E_{[x, 0]}\left[E_{\left[X_{\tau}, N_{\tau}\right]}\left[\widehat{f \cdot \lambda}\left(Y_{t-\tau}\right)\right] ; \tau \leqq t\right] \\
= & \int_{0}^{t} \int_{\hat{S}} P_{[x, 0]}\left(\tau \in d s, X_{\tau} \in d \boldsymbol{y}, N_{\tau}=\boldsymbol{p}\right) \lambda^{|\boldsymbol{p}|} \hat{u}(t-s, \cdot)(\boldsymbol{y}) \\
= & \int_{0}^{t} \int_{S} K([x, 0] ; d s,[d y, p]) \lambda^{p} \sum_{n \neq 1} \frac{q_{n}(y)}{k(x)} u(t-s, y)^{n} \\
= & \int_{0}^{t} \int_{S} K([x, 0] ; d s,[d y, p]) \lambda^{p} F(y ; u(t-s, y)) .
\end{aligned}
$$

Now (3. 18) and (3. 19) prove the lemma.

Q.E.D.

Next, we shall prove

Lemma 3. 2. Let $T_{t}^{J}$ be the semi-group on $\boldsymbol{B}(S)$ induced by the non-branching part $Y_{t}^{0}$ of a branching Markov process with age $Y_{t}$ satisfying Condition 1 and let $H_{t}$ be the semi-group on $\boldsymbol{B}(E)$ induced by the basic Markov process $X_{t}^{\prime}$ of $Y_{t}$. Then we have

$$
T_{t} \widehat{f \cdot 2}([x, 0])=H_{t} f(x), \quad f \in C(E), x \in E .
$$

Proof. Using (3.7) and (3.11), we can see that

14) cf. (2. 5). 


$$
\begin{aligned}
P_{[x, 0]}^{0}\left(X_{t}^{0} \in A, N_{t}^{0}=p\right) & =P_{[x, 0]}\left(X_{t} \in A, \sigma_{p} \leqq t<\sigma_{p+1} \wedge \tau\right) \\
& =E_{x}\left[e^{-2 \int_{0}^{t} k\left(X_{s}^{\prime}\right) d s} \frac{\left(\int_{0}^{t} k\left(X_{s}^{\prime}\right) d s\right)^{p}}{p !} I_{A}\left(X_{t}^{\prime}\right)\right],
\end{aligned}
$$

for any $A \in \mathscr{B}(E)$. Consequently, we have

$$
\begin{aligned}
T_{t}^{0} \widehat{f \cdot \lambda}([x, 0]) & =\sum_{p=0}^{\infty} E_{[x, 0]}\left[\lambda^{p} f\left(X_{t}\right) ; N_{t}^{0}=p\right] \\
& =\sum_{p=0}^{\infty} E_{x}\left[e^{-2 \int_{0}^{t} k\left(X_{s}^{\prime}\right) d s} \frac{\left(\lambda \int_{0}^{t} k\left(X_{s}^{\prime}\right) d s\right)^{p}}{p !} f\left(X_{t}^{\prime}\right)\right] \\
& =E_{x}\left[e^{-(2-\lambda) \int_{0}^{t} k\left(X_{s}^{\prime}\right) d s} f\left(X_{t}^{\prime}\right)\right], \quad f \in C(E) .
\end{aligned}
$$

If we put $\lambda=2$, then (3.20) follows from (3.21) immediately. Q.E.D.

Now let $H_{t}$ be the semi-group on $\boldsymbol{B}(E)$ and $F$ be the function given in (3. 14). For a given system $\left(H_{t}, k, F\right)$, consider the following equation:

$$
\begin{array}{r}
u(t, x)=H_{t} f(x)+\int_{0}^{t} H_{s}(k(\cdot) F(\cdot ; u(t-s, \cdot)))(x) d s, \\
f \in C(E), x \in E, 0 \leqq t \leqq T,
\end{array}
$$

where $T$ is a positive constant.

Then we have

Theorem 3. 1. Let $T_{t}$ be the semi-group on $\boldsymbol{B}(\hat{\boldsymbol{S}})$ induced by a branching Markov process with age $Y_{t}$ on $\boldsymbol{S}$ satisfying Condition 1 and let $H_{t}$ be the semi-group on $\boldsymbol{B}(E)$ induced by the basic Markov process $X_{t}^{\prime}$ of $Y_{t}$. Further for, $f \in \boldsymbol{C}(E)$, set

$$
u(t, x)=\left.\left(T_{t} \widehat{f \cdot 2}\right)\right|_{E}(x), \quad x \in E .
$$

If $u(t, x)$ is finite for any $x \in E$ and $0 \leqq t \leqq T$, then it satisfies (3.22).

Proof. It follows from (3. 13) that

$$
\begin{aligned}
& \int_{0}^{t} \int_{S} K([x, 0] ; d s,[d y, p]) 2^{p} F(y ; u(t-s, y)) \\
= & \int_{0}^{t} \sum_{p=0}^{\infty} 2^{p} E_{x}\left[e^{-2 \int_{0}^{s} k\left(X_{v}^{\prime}\right) d v} \frac{\left(\int_{0}^{s} k\left(X_{v}^{\prime}\right) d v\right)^{p}}{p !} k\left(X_{s}^{\prime}\right) F\left(X_{s}^{\prime} ; u\left(t-s, X_{s}^{\prime}\right)\right)\right] d s \\
= & \int_{0}^{t} E_{x}\left[k\left(X_{s}^{\prime}\right) F\left(X_{s}^{\prime} ; u\left(t-s, X_{s}^{\prime}\right)\right)\right] d s \\
= & \int_{0}^{t} H_{s}(k(\cdot) F(\cdot ; u(t-s, \cdot)))(x) d s .
\end{aligned}
$$


Then the theorem follows from Lemma 3. 1, Lemma 3.2 and the above equation.

Q.E.D.

Remark 2. For any $f \in C^{*}(E)$, there exists $\varepsilon>0$ depending on $\|f\|$ such that $\left.\left(T_{t} \widehat{f \cdot 2}\right)\right|_{E}(x)$ is finite for $t \in[0, \varepsilon)$ (cf. Nagasawa [13], Proposition 5. 16 and also, for special cases, see Lemma 6.1).

§4. Signed branching Markov process with age. We have considered the case where $q_{n}(x) \geqq 0$ and $q_{1}(x)=0$ in the last section. In this section, we shall remove such restrictions.

Let $\left\{\left(q_{n}^{+}(x), q_{n}^{-}(x)\right) ; n=0,1,2, \cdots\right\}$ be a system of pairs of non-negative bounded continuous functions on $E$ such that

$$
q_{n}^{+}(x) q_{n}^{-}(x)=0, \quad n=0,1,2, \cdots
$$

Further let $k(x)$ defined by

$$
k(x)=\sum_{n=0}^{\infty}\left\{q_{n}^{+}(x)+q_{n}^{-}(x)\right\}
$$

be a non-negative bounded continuous function on $E$. Then we shall define the system $\{\pi(\cdot, \cdot)\}$ by

$$
\begin{aligned}
& \pi([x, p, 0],[B, \boldsymbol{q}, 1])=\pi([x, p, 1],[B, \boldsymbol{q}, 0]) \\
= & \pi([x, p, 2],[B, \boldsymbol{q}, 3])=\pi([x, p, 3],[B, \boldsymbol{q}, 2]) \\
= & \sum_{n=0}^{\infty} \frac{q_{n}^{+}(x)}{k(x)} \delta_{n}([x, p],[B, \boldsymbol{q}]),
\end{aligned}
$$

$$
\begin{aligned}
& \pi([x, p, 0],[B, \boldsymbol{q}, 3])=\pi([x, p, 3],[B, \boldsymbol{q}, 0]) \\
= & \pi([x, p, 1],[B, \boldsymbol{q}, 2])=\pi([x, p, 2],[B, \boldsymbol{q}, 1]) \\
= & \sum_{n=0}^{\infty} \frac{q_{n}^{-}(x)}{k(x)} \delta_{n}([x, p],[B, \boldsymbol{q}]), \\
& \pi\left([x, p, j],\left[B, \boldsymbol{q}, j^{\prime}\right]\right)=0 \text { for the other pairs of }\left(j, j^{\prime}\right), \\
& \quad[x, p] \in S, j, j^{\prime} \in J,[B, \boldsymbol{q}] \in \mathscr{B}(\hat{S}),
\end{aligned}
$$

where $\delta_{n}$ is defined by

$$
\delta_{n}([x, p],[B, \boldsymbol{q}])= \begin{cases}1, & \text { if } \boldsymbol{x}=[x, x, \cdots, x] \in B \cap E^{n},|\boldsymbol{q}|=p, n \geqq 0, \\ 0, & \text { otherwise. }\end{cases}
$$


For a given system $\left\{\left(q_{n}^{+}(x), q_{n}^{-}(x)\right) ; n=0,1,2, \cdots\right\}$ and $k(x)=\sum_{n=0}^{\infty}\left(\dot{q}_{n}^{+}(x)+q_{n}^{-}(x)\right)$, let us consider a signed branching Markov process with age $Z=\left\{Z_{t}=\left[X_{t}\right.\right.$, $\left.\left.N_{t}, J_{t}\right], \zeta, \mathscr{B}_{t}, P_{[\boldsymbol{x}, \boldsymbol{p}, j]} ;[\boldsymbol{x}, \boldsymbol{p}, j] \in \tilde{\boldsymbol{S}}\right\}$ on $\tilde{\boldsymbol{S}}$ satisfying the following condition.

Condition 2. (i) For any fixed $j \in J$, the process $\left\{Y_{t}^{(j)}=\left[X_{t}, N_{t}\right], \zeta, \mathscr{B}_{t}\right.$, $\left.P_{[x, p, j]} ;\{x, p] \in S\right\}$ is a strong Markov process on $\hat{S}$ and it satisfies (i) and (ii) of Condition 1 for given $k(x)$, but where $\sigma$, and $\tau$ for $Y_{t}^{(j)}$ are replaced by $\sigma$ and $\eta$ for $Z$. Also $Y_{t}^{(j)}, j \in J$, are stochastically equivalent to each other.

(ii) It holds that for any $\alpha>0$

$$
\begin{aligned}
& E_{[x, p, j]}\left[e^{-\alpha \eta} ;\left[X_{\eta}, N_{\eta}, J_{\eta}\right] \in\left[B, \boldsymbol{q}, j^{\prime}\right]\right] \\
= & E_{[x, p, j]}\left[e^{-\alpha \eta} \pi\left(\left[X_{\eta-}, N_{\eta_{-}}, J_{\eta_{-}}\right],\left[B, \boldsymbol{q}, j^{\prime}\right]\right)\right],
\end{aligned}
$$

and

$$
\begin{aligned}
& E_{[x, 0, j]}\left[e^{-\alpha \sigma} ;\left[X_{\sigma}, N_{\sigma}, J_{\sigma}\right] \in[A, q, j]\right] \\
= & E_{[x, 0, j]}\left[e^{-\alpha \sigma} \delta_{\left[X_{\sigma-}, N_{\sigma-+}\right]}([A, q])\right], \\
& j, j^{\prime} \in J,[x, p] \in S,[B, \boldsymbol{q}] \in \mathscr{B}(\hat{\boldsymbol{S}}), A \in \mathscr{B}(E),
\end{aligned}
$$

where $E_{[x, p, j]}$ denotes the integral by $P_{[x, p, j]}, \pi$ is given in $(4.1)$ and $\delta_{[x, p]}(\cdot)$ denotes the $\delta$-measure assigned to $[x, p]$.

The existence of a signed branching Markov process with age satisfying condition 2 will be shown in $\S 8$.

(i) of the condition states that two processes satisfying Condition 1 and 2 have the same character until their first branching, while (ii) gives the new branching law attached to the new space $\widetilde{\boldsymbol{s}}$.

Similarly as in the case of a branching Markov process with age, we shall give the following

Definition 4. 1. The process $X^{\prime}$. considered in (i) of Condition 2 (or (ii) of Condition 1) is called the basic Markov process of $Z$.

Let us set

$$
Z_{t}^{0}(w)=\left\{\begin{array}{cl}
Z_{t}(w), & \text { if } t<\eta(w) \\
\Delta, & \text { if } t \geqq \eta(w) .
\end{array}\right.
$$


Then the probability measure for $Z_{t}^{0}$ is denoted by $P_{[x, p, j]}^{0}$ and the integral by $P_{[\boldsymbol{x}, \boldsymbol{p}, j]}^{0}$ is denoted by $E_{[\boldsymbol{x}, \boldsymbol{p}, j]}^{0}$. The semi-group induced by $Z_{t}^{0}$ is denoted by $U_{t}^{0}$. . Then we have

$$
\begin{gathered}
U_{t}^{0} h([\boldsymbol{x}, \boldsymbol{p}, j])=E_{[\boldsymbol{x}, \boldsymbol{p}, \boldsymbol{j}]}^{0}\left[h\left(Z_{t}\right)\right]=E_{[\boldsymbol{x}, \boldsymbol{p}, j]}\left[h\left(Z_{t}\right) ; t<\eta\right], \\
{[\boldsymbol{x}, \boldsymbol{p}, j] \in \widetilde{\boldsymbol{S}}, h \in \boldsymbol{B}(\widetilde{\boldsymbol{S}}) .}
\end{gathered}
$$

Definition 4. 2. When we restrict the starting point of $Z_{t}^{0}$ on $S \times J$, $Z_{t}^{0}$ is called the non-branching part of $Z$.

Now we shall define $K$ and $F$ as follows:

$$
\begin{gathered}
K([x, p, j] ; d s,[A, q, j])=P_{[x, p, j]}\left(\ddot{\eta} \in d s, Z_{\eta_{-}} \in[A, q, j]\right), \\
{[x, p] \in S, j \in J,}
\end{gathered}
$$

and

$$
F(x ; \xi)=\sum_{n=0}^{\infty} \frac{\left\{q_{n}^{+}(x)-q_{n}^{-}(x)\right.}{k(x)} \xi^{n}, \quad x \in E, \quad \xi \in R^{1}
$$

Then $K([x, p, j] ; \cdot, \cdot)$ is a measure on $\mathscr{B}([0, \infty) \times(S \times J))$ : and it follows from (i) of Condition 2 and (3.13) that

$$
\begin{gathered}
K([x, 0, j] ; d s,[A, p, j])=E_{x}\left[e^{-2 \int_{0}^{t} k\left(X_{s}^{\prime}\right) d s} \frac{\left(\int_{0}^{t} k\left(X_{s}^{\prime}\right) d s\right)^{p}}{p !} k\left(X_{t}^{\prime}\right) I_{\dot{A}}\left(X_{t}^{\prime}\right) d t\right], \\
A \in \mathscr{B}(E) !
\end{gathered}
$$

where $E_{x}$ denotes the integral by the probability measure $P_{x}$ of the basic Markov process $X^{\prime}$. ' Also, we can see that (3. 7)-(3. 11) hold if we replace $\tau$ by $\eta$. Then we have

Lemma 4. 1. Let $U_{t}^{0}$ be the semi-group on $\boldsymbol{B}(S \times J)$ induced by the nonbranching part $Z_{t}^{0}$ of a signed branching Markov process with age $Z_{i}$ satisfying Condition 2 and let $H_{t}$ be the semi-group on $\boldsymbol{B}(E)^{-}$induced by the basic Markov process $X_{t}^{\prime}$ of $Z_{t}$. Then we have

$$
U_{t}^{0} \widetilde{f: 2}([x, 0,0])=H_{t} f(x) ; . \quad f \in \boldsymbol{C}(E), \quad x \in E_{i},
$$

Now, for a given system $\left(U_{i}^{0}, K, F\right)$, consider the following equation:

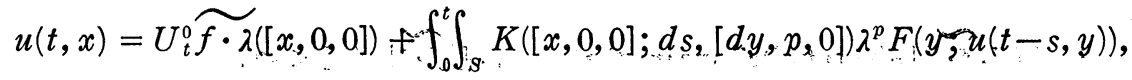

$$
\begin{aligned}
& f \in \boldsymbol{C}(E), 0 \leqq \lambda, 0 \leqq t \leqq T, x \in E,
\end{aligned}
$$


where $T$ is a positive constant.

Then we have

Lemma 4. 2. Let $U_{t}$ be the semi-group on $\boldsymbol{B}(\widetilde{\mathbf{S}})$ induced by a signed branching Markov process with age $Z_{t}$ on $\tilde{\boldsymbol{S}}$ satisfying Condition 2 and let $U_{t}^{0}$ be the semi-group on $\boldsymbol{B}(S \times J)$ induced by the non-branching part $Z_{t}^{0}$ of $Z_{t}$. Let also

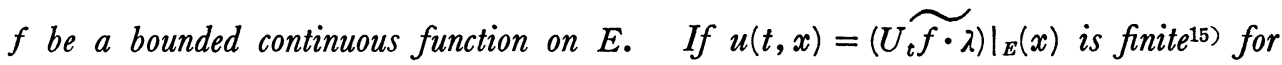
any $x \in E$ and $0 \leqq t \leqq T$, then $u(t, x)$ satisfies (4.9).

Proof. Let us assume that $u(t, x)$ is finite for $x \in E$ and $0 \leqq t \leqq T$. By the strong Markov property of $Z_{t}$, it holds that

$$
\left(\left.U_{t} \widetilde{f \cdot \lambda}\right|_{E}(x)=E_{[x, 0,0]}\left[\widetilde{f \cdot \lambda}\left(Z_{t}\right) ; t<\eta\right]+E_{[x, 0,0]}\left[U_{t-\eta} \widetilde{f \cdot \lambda\left(Z_{\eta}\right)} ; \eta \leqq t\right] .\right.
$$

On the other hand, we have from the signed branching property (2.13) of $Z_{t}$

$$
U_{t} \widetilde{f \cdot \lambda}([\boldsymbol{x}, \boldsymbol{p}, j])=(-1)^{\left[\frac{j}{2}\right]} \lambda^{|\boldsymbol{p}|} \hat{u}(t, \cdot)([\boldsymbol{x}]),
$$

and hence, by (4.2), (4.5) and (4.6), we have

$$
\begin{aligned}
& E_{[x, 0,0]}\left[U_{t-\eta} \widetilde{f \cdot \lambda}\left(Z_{\eta}\right) ; \eta \leqq t\right] \\
= & \int_{0}^{t} \int_{\tilde{\mathbf{s}}} P_{[x, 0,0]}\left(\eta \in d s, Z_{\eta} \in[d \boldsymbol{y}, \boldsymbol{p}, j]\right) U_{t-s} \widetilde{f \cdot \lambda}([\boldsymbol{y}, \boldsymbol{p}, j])^{16)} \\
= & \int_{0}^{t} \int_{S} K([x, 0,0] ; d s,[d y, p, 0]) \lambda^{p}\left\{\sum_{n=0}^{\infty} \frac{q_{n}^{+}(y)}{k(y)} u(t-s, y)^{n}-\sum_{n=0}^{\infty} \frac{q_{n}^{-}(y)}{k(y)} u(t-s, y)^{n}\right\} \\
= & \int_{0}^{t} \int_{S} K([x, 0,0] ; d s,[d y, p, 0]) \lambda^{p} F(y ; u(t-s, y)) .
\end{aligned}
$$

Thus the lemma is obtained from (4.10) and the above equation. Q.E.D.

Now let $H_{t}$ be the semi-group on $\boldsymbol{B}(E)$ and $F$ be the function given in (4.6). For a given system $\left(H_{t}, k, F\right)$, we consider the following equation

$$
\begin{array}{r}
u(t, x)=H_{t} f(x)+\int_{0}^{t} H_{s}(k(\cdot) F(\cdot ; u(t-s, \cdot)))(x) d s, \\
f \in C(E), x \in E, 0 \leqq t \leqq T,
\end{array}
$$

15) “( $\left.\left(U_{t} \widetilde{f} \cdot \lambda\right)\right|_{E}(x)$ is finite" means that $E_{[x, 0,0]}\left[\mid \widetilde{f \cdot \lambda \mid}\left(Z_{t}\right)\right]<\infty$.

16) cf. Remark 1 in $\S 3$. 
where $T$ is a positive constant. Then we have

Theorem 4. 1. Let $U_{t}$ be the semi-group on $\boldsymbol{B}(\widetilde{\mathbf{S}})$ induced by a signed branching Markov process with age $Z_{t}$ on $\tilde{\boldsymbol{S}}$ satisfying Condition 2 and let $H_{t}$ be the semi-group on $\boldsymbol{B}(E)$ induced by the basic Markov process $X_{t}^{\prime}$ of $Z_{t}$. Further, for $f \in C(E)$, set

$$
u(t, x)=\left.\left(U_{t} \widetilde{f \cdot 2}\right)\right|_{E}(x), \quad x \in E .
$$

If $u(t, x)$ is finite for any $x \in E$ and $0 \leqq t \leqq T$, then it satisfies (4. 11).

Proof. By the same method as in the proof of Theorem 3.1, we have from (4. 7)

$$
\begin{aligned}
& \int_{0}^{t} \int_{S} K([x, 0,0] ; d s,[d y, p, 0]) 2^{p} F(y ; u(t-s, y)) \\
= & \int_{0}^{t} \int_{S} H_{s}(k(\cdot) F(\cdot ; u(t-s, \cdot)))(x) d s .
\end{aligned}
$$

Then the theorem follows from Lemma 4.1 and Lemma 4.2. Q.E.D.

Now let $E=R^{d} \cup\{\infty\}$ be the space obtained by the one-point compactification of $R^{d}$ and consider the standard Brownian motion $X^{\prime}$ on $R^{d}$. Considering the point $\infty$ is the trap of $X^{\prime}$, the process $X^{\prime}$ can be regarded as the process on $E$. Then we can consider a signed branching Markov process with age $Z_{t}$ on $\tilde{\boldsymbol{S}}$ corresponding to the basic Markov process $X^{\prime}$. But when we take a starting point of $Z_{t}$ in $R^{d}$ a branching law at $\infty$ is not needed because almost all sample paths do not reach $\infty$. Hence it is sufficient in the present case that $q_{n}^{+}(x), q_{n}^{-}(x)$ and $k(x)$ are bounded and continuous in $R^{d}$, and we may consider $q_{n}^{+}(\infty)=q_{n}^{-}(\infty)=k(\infty)=0$.

Remark 1. For the case stated above, Theorem 4. 1 holds for $f \in C\left(R^{d}\right)$ with $f(\infty)=0$. The proof is given as follows: let $\rho_{n}(x), n \geqq 1$, be bounded continuous functions such that

$$
\begin{cases}\rho_{n}(x)=1, & \text { if }\|x\|<n \\ 0 \leqq \rho_{n}(x) \leqq 1, & \text { if } n \leqq\|x\| \leqq n+1, \\ \rho_{n}(x)=0, & \text { if }\|x\|>n+1 \text { or } x=\infty .\end{cases}
$$

Then $\rho_{n} f \in C(E)$. On the other hand, if $U_{t} \widetilde{f \cdot \lambda}$ is finite ${ }^{17)}$ we have by Lebesgue's convergence theorem

17) cf. Foot-note 10). 


$$
U_{t} \widetilde{f \cdot \lambda}([\boldsymbol{x}, \boldsymbol{p}, j])=\lim _{n \rightarrow \infty} U_{t} \widetilde{\rho_{n} f \cdot \lambda}([\boldsymbol{x}, \boldsymbol{p}, j]), \quad[\boldsymbol{x}, \boldsymbol{p}, j] \in \tilde{\mathbf{S}} .
$$

Hence it follows from (2. 13)

$$
U_{t} \widetilde{f \cdot \lambda}=\left(U_{t} \widetilde{f \cdot \lambda)\left.\right|_{E} \cdot \lambda}\right.
$$

provided each side is finite, because it holds for $\rho_{n} f$. Then we can see, as in the proof of Lemma 4.2, that Lemma 4. 2 holds for $f \in C\left(R^{d}\right)$, with $f(\infty)=0$. Evidently Lemma 4.1 holds for our $f$ and accordingly we can see that Theorem 4.1 holds for our $f$.

Corollary 4. 1. Let $Z_{t}$ be a signed branching Markov process with age satisfying Condition 2 whose basic Markov process is a standard Brownian motion on $R^{d}$ and let $U_{t}$ be the semi-group on $\boldsymbol{B}(\widetilde{\mathbf{S}})$ induced by $Z_{t} . \quad$ Let us assume that $k(x) F(x ; \xi)$ satisfies Lipschitz's condition:

$$
\begin{array}{r}
\left|k\left(x_{1}\right) F\left(x_{1} ; \xi_{1}\right)-k\left(x_{2}\right) F\left(x_{2} ; \xi_{2}\right)\right| \leqq K\left\{\left\|x_{1}-x_{2}\right\|+\left|\xi_{1}-\xi_{2}\right|\right\}, \\
x_{1}, x_{2} \in R^{d}, \xi_{1}, \xi_{2} \in R^{1},
\end{array}
$$

where $K$ is a positive constant and $\left\|x_{1}-x_{2}\right\|$ denotes the Euclidian distance between $x_{1}$ and $x_{2}$. If, for $f \in C(E), u(t, x)=\left.\left(U_{t} \widetilde{f \cdot 2}\right)\right|_{E}(x)$ is bounded for any $x \in R^{d}$ and $0 \leqq t<T$, then $u(t, x)$ is the bounded solution of parabolic equation

$$
\frac{\partial u(t, x)}{\partial t}=\frac{1}{2} \Delta u(t, x)+k(x) F(x ; u(t, x)), \quad x \in R^{d}, 0<t<T,
$$

with the initial condition $u(0+, x)=f(x)$.

Now we shall give a simple remark on a signed branching Markov process with age.

Remark 2. Let $Z_{t}$ be a signed branching Markov process with age on $\tilde{\boldsymbol{S}}$ satisfying Condition 2 and let $U_{t}$ be the semi-group induced by $Z_{t}$. If, for instance, we replace $\pi$ in (4.2) by $\pi_{1}$ defined by

$$
\begin{aligned}
& \pi_{1}([x, p, 0],[B, \boldsymbol{q}, 1])=\pi_{1}([x, p, 1],[B, \boldsymbol{q}, 0]) \\
= & \pi_{1}([x, p, 2],[B, \boldsymbol{q}, 3])=\pi_{1}([x, p, 3],[B, \boldsymbol{q}, 2]) \\
= & \sum_{n=0}^{\infty} \frac{q_{n}^{+}(x)}{k(x)} \delta_{n}([x, p],[B, \boldsymbol{q}])(=\pi([x, p, 0],[B, \boldsymbol{q}, 1])), \\
& \pi_{1}([x, p, 0],[B, \boldsymbol{q}, 2])=\pi_{1}([x, p, 2],[B, \boldsymbol{q}, 0])
\end{aligned}
$$




$$
\begin{aligned}
= & \pi_{1}([x, p, 1],[B, \boldsymbol{q}, 3])=\pi_{1}([x, p, 3],[B, \boldsymbol{q}, 1]) \\
= & \sum_{n=0}^{\infty} \frac{q_{n}^{-}(x)}{k(x)} \delta_{n}([x, p],[B, \boldsymbol{q}])(=\pi([x, p, 0],[B, \boldsymbol{q}, 3]), \\
& \pi_{1}\left([x, p, j],\left[B, \boldsymbol{q}, j^{\prime}\right]\right)=0 \quad \text { for the other pairs of }\left(j, j^{\prime}\right), \\
& {[x, p] \in S, j, j^{\prime} \in J,[B, \boldsymbol{q}] \in \mathscr{B}(\boldsymbol{S}), }
\end{aligned}
$$

then we have a new process $Z_{t}^{\prime}$ and the corresponding semi-group $U_{t}^{\prime}$. Evidently $Z_{t}^{\prime}$ is not stochastically equivalent to $Z_{t}$, but it holds that $U_{t} \widetilde{f \cdot \lambda}=U_{t}^{\prime} \widetilde{f \cdot \lambda}$ for any $f \in \boldsymbol{C}(E)$ provided each side exist. Therefore $U_{t} \widetilde{f \cdot \lambda}=U_{t}^{\prime} \widetilde{f \cdot \lambda}$ does not imply the stochastic equivalence of the processes $Z_{t}$ and $Z_{t}^{\prime}$.

§5. A sufficient condition. Let $Z=\left\{Z_{t}=\left[X_{t}, N_{t}, J_{t}\right], \zeta, \mathscr{B}_{t}\right.$, $\left.P_{[\boldsymbol{x}, \boldsymbol{p}, j]} ;[\boldsymbol{x}, \boldsymbol{p}, j] \in \widetilde{\boldsymbol{S}}\right\}$ be a strong Markov process on $\widetilde{\boldsymbol{S}}$ which is not assumed $a$ priori to be a signed branching Markov process with age. In this section, we shall give a sufficient condition which makes the process $Z_{t}$ on $\widetilde{\boldsymbol{S}}$ a signed branching Markov process with age on $\tilde{\boldsymbol{S}}$.

Now let us define $U_{t}^{0}$ and $\Psi$ by

$$
\begin{gathered}
U_{t}^{0} h([\boldsymbol{x}, \boldsymbol{p}, j])=E_{[\boldsymbol{x}, \boldsymbol{p}, j]}\left[h\left(Z_{t}\right) ; t<\eta\right], \\
\Psi\left([\boldsymbol{x}, \boldsymbol{p}, j] ; d s, \quad\left[B, \boldsymbol{p}^{\prime}, j^{\prime}\right]\right)=P_{[\boldsymbol{x}, \boldsymbol{p}, j]}\left(\eta \in d s, Z_{\eta} \in\left[B, \boldsymbol{p}^{\prime}, j^{\prime}\right]\right), \\
\quad[\boldsymbol{x}, \boldsymbol{p}, j] \in \widetilde{\mathbf{S}},\left[B, \boldsymbol{p}^{\prime}, j^{\prime}\right] \in \mathscr{B}(\widetilde{\boldsymbol{S}}),
\end{gathered}
$$

where $h$ is a Borel measurable function on $\tilde{\mathbf{S}}, \eta(w)=\inf \left\{t>0 ; J_{t}(w) \neq J_{0}(w)\right.$ or $\left.\sup _{s \leq t}\left|N_{s}(w)\right|=\infty\right\}$ and $E_{[\boldsymbol{x}, \boldsymbol{p}, j]}$ denotes the integral by $P_{[\boldsymbol{x}, \boldsymbol{p}, j]} . \Psi([\boldsymbol{x}, \boldsymbol{p}, j]$; $\cdot, \cdot)$ is a measure on $\mathscr{B}([0, \infty) \times \widetilde{\boldsymbol{S}})$. Then we consider the following

Condition 3. (i) Condition 2 holds.

(ii)

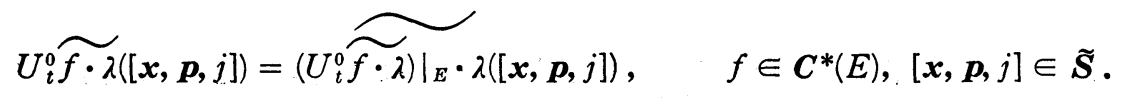

(iii) For $f \in \boldsymbol{B}^{*}([0 ; \infty) \times E)$, set

$$
\widetilde{f^{(t)} \cdot \lambda}=\widetilde{f(t, \cdot) \cdot \lambda}
$$


where $f(t, \cdot)$ denotes the function on $E$ for fixed $t \geqq 0$. Then it holds that for any $t \geqq 0, m \geqq n-1$

$$
\begin{aligned}
& \int_{0}^{t} \int_{S^{m} \times\left\{j^{\prime}\right\}} \Psi\left([\boldsymbol{x}, \boldsymbol{p}, j] ; d s,\left[d \boldsymbol{y}, \boldsymbol{q}, j^{\prime}\right]\right) \widetilde{f^{(s)} \cdot \lambda\left(\left[\boldsymbol{y}, \boldsymbol{q}, j^{\prime}\right]\right)} \\
& =\sum_{i=1}^{\infty} \int_{0}^{t} \int_{S^{m-n+1} \times\left\{j^{\prime}\right\}} \Psi\left(\left[x_{i}, p_{i}, j\right] ; d s,\left[d \boldsymbol{y}, \boldsymbol{p}^{\prime}, j^{\prime}\right]\right) \widetilde{f^{(s)} \cdot \lambda\left(\left[\boldsymbol{y}, \boldsymbol{p}^{\prime}, j^{\prime}\right]\right)}
\end{aligned}
$$

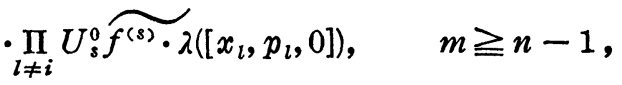

where $\boldsymbol{x}=\left[x_{1}, x_{2}, \cdots, x_{n}\right]$ and $\boldsymbol{p}=\left[p_{1}, p_{2}, \cdots, p_{n}\right]$.

(iv) $\Delta$ and $[\partial, p, j], p \in N$ and $j \in J$, are traps.

(v) Let

$$
\eta_{\infty}=\lim _{n \rightarrow \infty} \eta_{n}, \quad e_{\Delta}=\inf \left\{t>0 ; Z_{t}=\Delta\right\},
$$

where $\eta_{n}$ is given in (2. 7). Then it holds that

$$
P_{[x, p, j]}\left(\eta_{\infty}=e_{\Delta}, \eta_{\infty}<\zeta\right)=P_{[x, p, j]}\left(\eta_{\infty}<\zeta\right), \quad[x, p, j] \in \tilde{\boldsymbol{S}}
$$

(ii) requires the independence of the motions of $n$-particles starting from $\left[x_{1}, p_{1}, j\right],\left[x_{2}, p_{2}, j\right], \cdots,\left[x_{n}, p_{n}, j\right]$ and ending at the minimum of their first branching times inf $\left\{t>0 ; J_{t}(w) \neq J_{0}(w)\right.$ or $\left.\sup _{s \leq t}\left|N_{s}(w)\right|=\infty\right\}$, while (5. 4) means that only one of them branches at the first branching time $\eta$ and the others do not. ((ii) and (iii) of Condition 3 correspond to the property $B$ III in [7]. ) The existence of a strong Markov process satisfying Condition 3 will be proved in $\S 8$.

Now our purpose is to prove the following

TheORem 5. 1. If a strong Markov process $Z=\left\{Z_{t}=\left[X_{t}, N_{t}, J_{t}\right], \zeta, \mathscr{B}_{t}\right.$, $\left.P_{[\boldsymbol{x}, \boldsymbol{p}, j]} ;[\boldsymbol{x}, \boldsymbol{p}, j] \in \tilde{\mathbf{S}}\right\}$ on $\tilde{\boldsymbol{s}}$ satisfies Condition 3 , then $Z$ is a signed branching Markov process with age.

First we shall prepare some lemmas. Let $U_{t}$ be the semi-group on $\boldsymbol{B}(\widetilde{\mathbf{S}})$ induced by $Z_{t}$ and set

$$
U_{t}^{(r)} h([\boldsymbol{x}, \boldsymbol{p}, j])=E_{[\boldsymbol{x}, \boldsymbol{p}, j]}\left[h\left(Z_{t}\right) ; \eta_{r} \leqq t<\eta_{r+1}\right], r \geqq 0,[\boldsymbol{x}, \boldsymbol{p}, j] \in \widetilde{\boldsymbol{S}},
$$

for a Borel measurable function $h$ on $\widetilde{\boldsymbol{S}}$ provided that $E_{[x, p, j]}\left(h\left(Z_{t}\right)\right)$ exists. In the following lemmas, it is always assumed that Condition 3 holds, $f \in C^{*}(E)$ and $0 \leqq \lambda<1$. 
Lemma 5. 1. For any $r \geqq 0$, we have

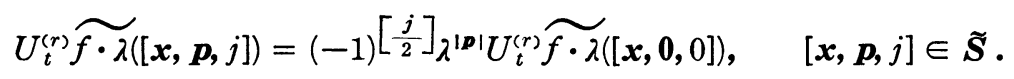

Proof. (5. 6) holds for $r=0$ and hence it suffices to prove (5. 6) for $r+1$ under the assumption that (5.6) holds for $r$.

Now it follows from (5.4) and (ii) of Condition 2 that for any $[\boldsymbol{x}, \boldsymbol{p}] \in \boldsymbol{S}$ and $[B, q] \in \mathscr{B}(\boldsymbol{S})$

$$
\begin{aligned}
& \Psi([\boldsymbol{x}, \boldsymbol{p}, 0] ; d t,[B, \boldsymbol{q}, 1])=\Psi([\boldsymbol{x}, \boldsymbol{p}, 1] ; d t,[B, \boldsymbol{q}, 0]) \\
= & \Psi([\boldsymbol{x}, \boldsymbol{p}, 2] ; d t,[B, \boldsymbol{q}, 3])=\Psi([\boldsymbol{x}, \boldsymbol{p}, 3] ; d t,[B, \boldsymbol{q}, 2]), \\
& \Psi(] \boldsymbol{x}, \boldsymbol{p}, 0] ; d t,[B, \boldsymbol{q}, 3])=\Psi([\boldsymbol{x}, \boldsymbol{p}, 3] ; d t,[B, \boldsymbol{q}, 0]) \\
= & \Psi([\boldsymbol{x}, \boldsymbol{p}, 1] ; d t,[B, \boldsymbol{q}, 2])=\Psi([\boldsymbol{x}, \boldsymbol{p}, 2] ; d t,[B, \boldsymbol{q}, 1]), \\
& \Psi\left([\boldsymbol{x}, \boldsymbol{p}, j] ; d t,\left[B, \boldsymbol{q}, j^{\prime}\right]\right)=0, \quad \text { for other pairs of }\left(j, j^{\prime}\right), \\
& \Psi\left([\boldsymbol{x}, \boldsymbol{p}, j] ; d t,\left[B, \boldsymbol{q}, j^{\prime}\right]\right)=\Psi\left([\boldsymbol{x}, \boldsymbol{0}, j] ; d t,\left[B, \boldsymbol{q}-\boldsymbol{p}, j^{\prime}\right]\right), \quad j, j^{\prime} \in J,
\end{aligned}
$$

where $\boldsymbol{q}-\boldsymbol{p}$ denotes $\boldsymbol{p}^{\prime}$ with $\left|\boldsymbol{p}^{\prime}\right|=|\boldsymbol{q}|-|\boldsymbol{p}|$. Then we can see from (5. 7) and the strong Markov property of $Z_{t}$ that

$$
\begin{aligned}
& U_{t}^{(r+1)} \widetilde{f} \cdot \lambda([\boldsymbol{x}, \boldsymbol{p}, j]) \\
= & \int_{0}^{t} \int_{\tilde{s}} \Psi\left([\boldsymbol{x}, \boldsymbol{p}, j] ; d s,\left[d \boldsymbol{y}, \boldsymbol{q}, j^{\prime}\right]\right) U_{t-s}^{(r)} \widetilde{f \cdot \lambda\left(\left[\boldsymbol{y}, \boldsymbol{q}, j^{\prime}\right]\right)} \\
= & \left.(-1)^{\left[\frac{j}{2}\right]}\right] \int_{0}^{t} \int_{\tilde{s}} \Psi\left([\boldsymbol{x}, \boldsymbol{0}, 0] ; d s,\left[d \boldsymbol{y}, \boldsymbol{q}, j^{\prime}\right]\right) U_{t-s}^{(r)} \widetilde{f} \cdot \lambda([\boldsymbol{y}, \boldsymbol{p}+\boldsymbol{q}, j]),
\end{aligned}
$$

where $|\boldsymbol{p}+\boldsymbol{q}|=|\boldsymbol{p}|+|\boldsymbol{q}|$. By the assumption of induction, we have from the above equation

$$
\begin{aligned}
& U_{t}^{(r+1)} \widetilde{f} \cdot \lambda([\boldsymbol{x}, \boldsymbol{p}, j]) \\
& =(-1)^{\left[\frac{j}{2}\right]} \lambda^{|\boldsymbol{p}|} \int_{0}^{t} \int_{\tilde{\boldsymbol{s}}} \Psi\left([\boldsymbol{x}, 0,0] ; d s,\left[\boldsymbol{d} \boldsymbol{y}, \boldsymbol{q}, j^{\prime}\right]\right) U_{t-s}^{(r)} \widetilde{f} \cdot \lambda\left(\left[\boldsymbol{y}, \boldsymbol{q}, j^{\prime}\right]\right) \\
& \left.=(-1)^{\left[\frac{j}{2}\right]}\right]_{\lambda^{|\boldsymbol{p}|} U_{t}^{(r+1)}} \widetilde{f \cdot \lambda([x, 0,0]),}
\end{aligned}
$$

as was to be proved.

Q.E.D.

Lemma 5. 2. For any $r \geqq 0$ and $[\boldsymbol{x}, \boldsymbol{p}]=\left[\left[x_{1}, x_{2}, \cdots, x_{n}\right],\left[p_{1}, p_{2}, \cdots, p_{n}\right]\right]$, 
(5. 8)

$$
\begin{gathered}
\sum_{r_{1}+r_{2}+\cdots+r_{n}=r} \int_{0}^{t} \sum_{i=1}^{n} \int_{\tilde{s}} \Psi\left(\left[x_{i}, p_{i}, j\right] ; d s,\left[d \boldsymbol{y}, \boldsymbol{q}, j^{\prime}\right]\right) U_{t-s}^{\left(r_{i}\right)} \widetilde{f \cdot \lambda\left(\left[\boldsymbol{y}, \boldsymbol{q}, j^{\prime}\right]\right)} \\
\prod_{l \neq i} U_{S_{s}^{0}}^{0} U_{t-s}^{\left(r_{l}\right)} \widetilde{f \cdot \lambda}\left(\left[x_{l}, p_{l}, 0\right]\right) \\
=(-1)^{\left[\frac{j}{2}\right]} \sum_{r_{1}+r_{2}+\cdots+r_{n}=r+1} \prod_{i=1}^{n} U_{t}^{\left(r_{i}\right)} \widetilde{f \cdot \lambda}\left(\left[x_{i}, p_{i}, 0\right]\right) .
\end{gathered}
$$

Proof. According to (i) of Condition 2 and Lemma 5. 1, it holds that

$$
U_{s}^{0} U_{t-s}^{(r)} \widetilde{f \cdot \lambda}([x, p, 0])=\lambda^{p} U_{s}^{0} U_{t-s}^{(r)} \widetilde{f \cdot \lambda}([x, 0,0]), \quad[x, p] \in S .
$$

Hence, by (5. 7) and Lemma 5. 1, it suffices to prove (5.8) for the case $\boldsymbol{p}=\mathbf{0}$ and $j=0$.

Now let us put $g^{\left(r_{i}\right)}(s)=U_{s}^{0} U_{t-s}^{\left(r_{i}\right)} \widetilde{f \cdot \lambda}\left(\left[x_{i}, 0,0\right]\right)$. Then $g^{(0)}(s)$ is indépendent of $s$ by the semi-group property of $U_{t}^{0}$. Further we can see from the strong Markov property of $Z_{t}$ that for $r \geqq 1$

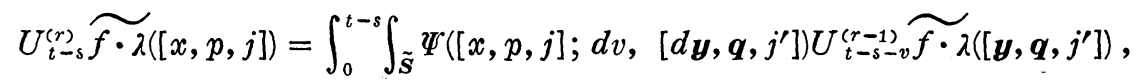

and hence we have

$$
g^{\left(r_{i}\right)}(s)=\int_{s}^{t} \int_{\tilde{s}} \Psi\left(\left[x_{i}, 0,0\right] ; d v,\left[d \boldsymbol{y}, \boldsymbol{q}, j^{\prime}\right]\right) U_{t-v}^{\left(r_{i}-1\right)} \widetilde{f \cdot \lambda}\left(\left[\boldsymbol{y}, \boldsymbol{q}, j^{\prime}\right]\right)
$$

Then the left hand side of (5.8), where $\boldsymbol{p}=\mathbf{0}$ and $j=0$, is equal to

$$
\sum_{r_{1}+r_{2}+\cdots+r_{n}=r} \int_{0}^{t} \sum_{i=1}^{n} d_{s}\left(-g^{\left(r_{i}+1\right)}(s)\right) \prod_{l \neq i} g^{\left(r_{l}\right)}(s) \text {. }
$$

Writing $r_{i}+1$ as $r_{i}$ and noting $d_{s} g^{(0)}(s)=0$ and $g^{\left(r_{i}\right)}(t)=0$ for $r_{i} \geqq 1$, the above expression is equal to

$$
\begin{aligned}
& \sum_{r_{1}+r_{2}+\cdots+r_{n}=r+1} \int_{0}^{t} \sum_{i=1}^{n} d_{s}\left(-g^{\left(r_{i}\right)}(s)\right) \prod_{l \neq i} g^{\left(r_{l}\right)}(s) \\
= & \sum_{r_{1}+r_{2}+\cdots+r_{n}=r+1} \prod_{i=1}^{n} g^{\left(r_{i}\right)}(0)=\sum_{r_{1}+r_{2}+\cdots+r_{n}=r+1} \sum_{i=1}^{n} U_{t}^{\left(r_{i}\right)} \widetilde{f \cdot \lambda\left(\left[x_{i}, 0,0\right]\right),}
\end{aligned}
$$

as was to be proved.

Q:E.D.

LEMMA 5. 3. For any $r \geqq 0,[\boldsymbol{x}, \boldsymbol{p}]=\left[\left[x_{1}, x_{2}, \cdots, x_{n}\right],\left[p_{1}, p_{2}, \cdots, p_{n}\right]\right.$. and $j \in J$, we have 
(5. 9) $\quad U_{t}^{(r)} \widetilde{f \cdot \lambda}([\boldsymbol{x}, \boldsymbol{p}, j])=(-1)^{\left[\frac{j}{2}\right]} \sum_{r_{1}+r_{2}+\cdots+r_{n}=r} \prod_{i=1}^{n} U_{t}^{\left(r_{i}\right)} \widetilde{f \cdot \lambda}\left(\left[x_{i}, p_{i}, 0\right]\right)$,

and

$$
\begin{gathered}
\sum_{m=n-1}^{\infty} \int_{0}^{t} \int_{S^{m} \times J} \Psi\left([\boldsymbol{x}, \boldsymbol{p}, j] ; d s,\left[d \boldsymbol{y}, \boldsymbol{q}, j^{\prime}\right]\right) \\
\cdot\left\{(-1)\left[\frac{j^{\prime}}{2}\right] \sum_{r_{1}+r_{2}+\cdots+r_{m}=r} \prod_{i=1}^{m} U_{t-s}^{\left(r_{i}\right)} \widetilde{f \cdot \lambda}\left(\left[y_{i}, q_{i}, 0\right]\right)\right\} \\
=\sum_{r_{1}+r_{2}+\cdots+r_{n}=r} \int_{0}^{t} \sum_{i=1}^{n} \int_{\tilde{s}} \Psi\left(\left[x_{i}, p_{i}, j\right] ; d s,\left[d \boldsymbol{y}, \boldsymbol{p}^{\prime}, j^{\prime}\right]\right) U_{t-s}^{\left(r_{i}\right)} \widetilde{f \cdot \lambda}\left(\left[\boldsymbol{y}, \boldsymbol{p}^{\prime}, j^{\prime}\right]\right) \\
\prod_{l \neq i} U_{s}^{0} U_{t-s}^{\left(r_{l}\right)} \widetilde{f \cdot \lambda}\left(\left[x_{l}, p_{l}, 0\right]\right),
\end{gathered}
$$

where $\boldsymbol{y}=\left[y_{1}, y_{2}, \cdots, y_{m}\right]$ and $\boldsymbol{q}=\left[q_{1}, q_{2}, \cdots, q_{m}\right]$.

Proof. For $r=0$, (5. 9) follows from Lemma 5. 1, and (5. 10) follows from Lemma 5. 1 and (iii) of Condition 3. Hence we shall prove the validity of (5.9) and (5.10) for $r+1$ under the assumption that (5.9) and (5. 10) hold up to $r$. Further, by Lemma 5. 1, we may assume $\boldsymbol{p}=\mathbf{0}$ and $j=0$.

By Lemma 5. 2 and the assumption of induction, we have

$$
\begin{aligned}
& \sum_{r_{1}+r_{2}+\cdots+r_{n}=r+1} \prod_{i=1}^{n} U_{t}^{\left(r_{i}\right)} \widetilde{f \cdot \lambda}\left(\left[x_{i}, 0,0\right]\right) \\
& =\sum_{m=n-1}^{\infty} \int_{0}^{t} \int_{S^{m} \times J} \Psi([\boldsymbol{x}, 0,0] ; d s,[d \boldsymbol{y}, \boldsymbol{p}, j]) \\
& \cdot\left\{(-1)^{\left[\frac{j}{2}\right]} \sum_{r_{1}+r_{2}+\cdots+r_{m}=r} \prod_{i=1}^{m} U_{t-s}^{\left(r_{i}\right)} \widetilde{f} \cdot \lambda\left(\left[y_{i}, p_{i}, 0\right]\right)\right\} \\
& =\int_{0}^{t} \int_{\tilde{s}} \Psi([\boldsymbol{x}, \mathbf{0}, 0] ; d s,[d \boldsymbol{y}, \boldsymbol{p}, j]) U_{t-s}^{(r)} \widetilde{f} \cdot \lambda([\boldsymbol{y}, \boldsymbol{p}, j]) \\
& =U_{t}^{(r+1)} \widetilde{f \cdot \lambda([\boldsymbol{x}, \mathbf{0}, 0])} \text {. }
\end{aligned}
$$

So (5. 9) holds for $r+1$.

Now we note that for any $(m, m)$-matrix $A=\left(a_{i, j}\right)_{i, j=1}^{m}$

$$
\begin{aligned}
& \sum_{\pi} \prod_{i=1}^{m} a_{\pi(i), i}=\prod_{i=1}^{m}\left(\sum_{k=1}^{m} a_{k, i}\right)-\sum_{\left(k_{1}, k_{2}, \cdots, k_{m-1}\right)} \prod_{i=1}^{m}\left(\sum_{j=1}^{m-1} a_{k_{j}, i}\right) \\
+ & \sum_{\left(k_{1}, k_{2}, \cdots, k_{m-2}\right)} \prod_{i=1}^{m}\left(\sum_{j=1}^{m-2} a_{k_{j}, i}\right)-\cdots+(-1)^{m-1} \sum_{(k)} \prod_{i=1}^{m} a_{k, i},
\end{aligned}
$$


holds, where $\Sigma_{\pi}$ denotes the summation over all permutations $(\pi(1), \pi(2), \cdots$, $\pi(m))$ of $(1,2, \cdots, m)$ and $\Sigma_{\left(k_{1}, k_{2}, \cdots, k_{r}\right)}, r \leqq m-1$, denotes the summation over all $\left(k_{1}, k_{2}, \cdots, k_{r}\right)$ such that $1 \leqq k_{i} \leqq m$ and all $k_{i}$ are different. ${ }^{18)}$

Let $h_{i} \in \boldsymbol{B}^{*}([0, \infty) \times E), \quad i=1,2, \cdots, m$. Considering $\widetilde{h_{j}^{(s)} \cdot \lambda\left(\left[y_{i}, p_{i}, 0\right]\right)}$ in the place of $a_{j, i}$ in (5.11), we have for $[x]=\left[x_{1}, x_{2}, \cdots, x_{n}\right]$

$$
\int_{0}^{t} \int_{S^{m} x\{j\}} \Psi([\boldsymbol{x}, 0,0] ; d s,[d \boldsymbol{y}, \boldsymbol{p}, j])\left\{(-1)^{\left[\frac{j}{2}\right]} \sum_{\pi} \prod_{i=1}^{m} \widetilde{h_{\pi(i)}^{(s)}} \cdot \lambda\left(\left[y_{i}, p_{i}, 0\right]\right)\right\}
$$

$$
\begin{gathered}
\left.\left.=\sum_{\nu=0}^{m-1}(-1)^{\nu} \sum_{\left(k_{1}, k_{2}, \cdots, k_{m-\nu}\right)} \int_{0}^{t} \int_{S^{m} \times\{j\}} \Psi[\boldsymbol{x}, 0,0]\right) ; d s,[\boldsymbol{d} \boldsymbol{y}, \boldsymbol{p}, j]\right) \\
\cdot(-1)\left[\frac{j}{2}\right] \prod_{i=1}^{m}\left\{\sum_{r=1}^{m-\nu} \widetilde{\left.h_{k_{r}}^{(s)} \cdot \lambda\left(\left[y_{i}, p_{i}, 0\right]\right)\right\}} .\right.
\end{gathered}
$$

According to Lemma 5. 1, and (5.4), the right hand side of the above equation is equal to

$$
\begin{aligned}
& \sum_{\nu=0}^{m-1}(-1)^{\nu} \sum_{\left(k_{1}, k_{2}, \cdots, k_{m-\nu}\right)} \int_{0}^{t} \int_{S^{m} \times\{j\}} \Psi([\boldsymbol{x}, \boldsymbol{0}, 0] ; d s,[d \boldsymbol{y}, \boldsymbol{p}, j]) \\
& \overbrace{\cdot\left(\sum_{r=1}^{m-\nu} h_{k_{r}}^{(s)}\right) \cdot \lambda[\boldsymbol{y}, \boldsymbol{p}, j]} \\
& =\int_{0}^{t} \sum_{i=1}^{n} \int_{S m-n+1 \times\{j\}} \Psi\left(\left[x_{i}, 0,0\right] ; d s,[d \boldsymbol{y}, \boldsymbol{p}, j]\right)(-1)^{\left[\frac{j}{2}\right]}\left\{\sum_{\nu=0}^{m-1}(-1)^{\nu} \sum_{\left(k_{1}, k_{2}, \ldots, k_{m-\nu}\right)}\right.
\end{aligned}
$$

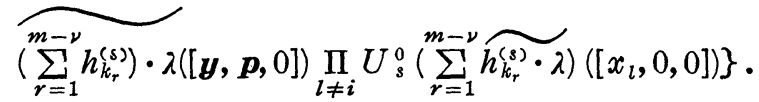

Now noting that for $\boldsymbol{y}=\left[y_{1}, y_{2}, \cdots, y_{m-n+1}\right], \boldsymbol{p}=\left[p_{1}, p_{2}, \cdots, p_{m-n+1}\right]$

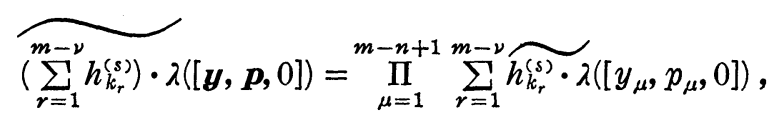

and applying again (5.11) to the integrand \{\} , the above expression is equal to

$$
\begin{aligned}
& \int_{0}^{t} \sum_{i=1}^{n} \int_{S^{m-n+1} \times\{j\}} \Psi\left(\left[x_{i}, 0,0\right] ; d s,[d \boldsymbol{y}, \boldsymbol{p}, j]\right)
\end{aligned}
$$

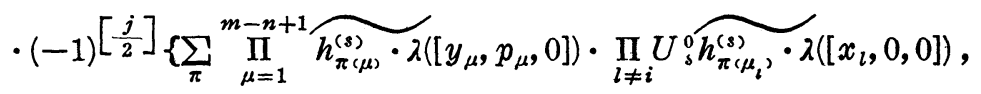

where $\left\{\mu_{l} ; 1 \leqq l \leqq n, l \neq i\right\}=\{m-n+2, m-n+3, \cdots, m\}$ and $\pi$ is a permutation on $(1,2, \cdots, m)$. If we use the following notations:

18) cf. Ryser [15], Th. 4.1 (p. 26) 
$\sum_{\left\langle k_{1}, k_{2}, \cdots, k_{m-n+1}\right\rangle}:$ the sum over all choices $\left(k_{1}, k_{2}, \cdots, k_{m-n+1}\right)$ from $(1,2, \cdots, m)$,

$\sum_{\pi}^{(k)} \quad:$ the sum over all permutations $\pi$ on $\left(k_{1}, k_{2}, \cdots, k_{m-n+1}\right)$,

$\sum_{\hat{\pi}}^{(\hat{k})} \quad:$ the sum over all permutations $\hat{\pi}$ on $\left(\hat{k}_{1}, \hat{k}_{2}, \cdots, \hat{k}_{n-1}\right)$ which is the remainder of $(1,2, \cdots, m)$ excluding $\left(k_{1}, k_{2}, \cdots, k_{m-n+1}\right)$,

then (5. 13) is equal to

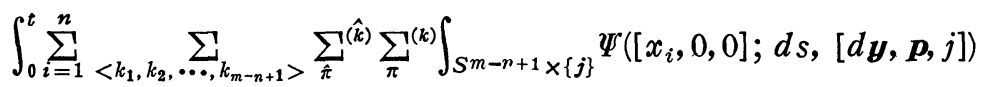

$$
\begin{aligned}
& \cdot(-1)^{\left[\frac{j}{2}\right]} \prod_{\mu=1}^{m-n+1} \overbrace{h_{k(\mu)}^{(s)}}^{m} \cdot \lambda\left(\left[y_{\mu}, p_{\mu}, 0\right]\right) \cdot \prod_{l \neq i} U_{s}^{0} \overbrace{h_{\hat{\pi}_{\left(\mu_{l}\right)}}^{(s)}} \cdot \lambda\left(\left[x_{l}, 0,0\right]\right) .
\end{aligned}
$$

Now putting $h_{k}^{(s)}=\left(\left.U_{t-s}^{(k)} \widetilde{f \cdot \lambda}\right|_{E}\right.$, we can see from (5.9) for $r_{i} \leqq r+1$

$$
\begin{aligned}
& \int_{0}^{t} \int_{S^{m} \times\{j\}} \Psi([\boldsymbol{x}, \mathbf{0}, 0] ; d s,[d \boldsymbol{y}, \boldsymbol{p}, j]) \\
& \cdot\left\{(-1)^{\left[\frac{j}{2}\right]} \sum_{r_{1}+r_{2}+\cdots+r_{m}=r+1} \prod_{i=1}^{m} U_{t-s}^{\left(r_{i}\right)} \widetilde{\left.f \cdot \lambda\left(\left[y_{i}, p_{i}, 0\right]\right)\right\}}\right. \\
& =\int_{0}^{t} \sum_{i=1}^{n} \sum_{r_{i}=0}^{r+1} \sum_{\hat{r}_{1}+\hat{r}_{2}+\ldots+\hat{r}_{n-1}=r+1-r_{i}} \int_{S m-n+1 \times\{j\}} \Psi\left(\left[x_{i}, 0,0\right] ; d s,[d \boldsymbol{y}, \boldsymbol{p}, j]\right) \\
& \cdot\left\{(-1)^{\left[\frac{j}{2}\right]} \sum_{r_{1}+r_{2}+\cdots+r_{m-n+1}=r_{i}} \prod_{\mu=1}^{m-n+1} U_{t-s}^{\left(r_{\mu}\right)} \widetilde{f \cdot \lambda}\left(\left[y_{\mu}, p_{\mu}, 0\right]\right)\right\} \\
& \cdot \prod_{l \neq i} U_{s}^{0} U_{t-s}^{\left.\hat{r}_{l}\right)} \widetilde{f \cdot \lambda}\left(\left[x_{l}, 0,0\right]\right) \\
& =\sum_{r_{1}+r_{2}+\cdots+r_{n}=r+1} \int_{0}^{t} \sum_{i=1}^{n} \int_{S m-n+1 \times\{j\}} \Psi\left(\left[x_{i}, 0,0\right] ; d s,[d \boldsymbol{y}, \boldsymbol{p}, j]\right) \\
& \cdot U_{t-s}^{\left(r_{i}\right)} \widetilde{f} \cdot \lambda([\boldsymbol{y}, \boldsymbol{p}, j]) \cdot \prod_{l \neq i} U_{s}^{0} U_{t-s}^{\left(r_{l}\right)} \widetilde{f \cdot \lambda}\left(\left[x_{l}, 0,0\right]\right) .
\end{aligned}
$$

Summing up both sides of the above equation over all $m \geqq n-1$, we have (5. 10) for $r+1$.

Q.E.D.

LEMMA 5. 4.

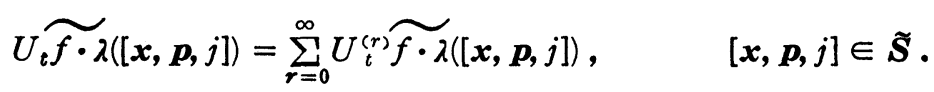

Proof. By (iv) and (v) of Condition 3, we have 


$$
\begin{aligned}
& \left.U_{t} \widetilde{f \cdot \lambda}[\boldsymbol{x}, \boldsymbol{p}, j]\right)=E_{[\boldsymbol{x}, \boldsymbol{p}, \boldsymbol{j}]}\left[\widetilde{f \cdot \lambda}\left(Z_{t}\right) ; t<\eta_{\infty}\right]+E_{[\boldsymbol{x}, \boldsymbol{p}, j]}\left[\widetilde{f \cdot \lambda}\left(Z_{t}\right) ; t \geqq \eta_{\infty}\right] \\
& =\sum_{r=0}^{\infty} E_{[x, p, j]}\left[\widetilde{f \cdot \lambda}\left(Z_{t}\right) ; \eta_{r} \leqq t<\eta_{r+1}\right]+E_{[x, p, j]}\left[U_{t-\eta_{\infty}} \widetilde{f \cdot \lambda(\Delta)} ; t \geqq \eta_{\infty}\right] \\
& =\sum_{r=0}^{\infty} U_{t}^{(r)} \widetilde{f \cdot \lambda([\boldsymbol{x}, \boldsymbol{p}, j])} \text {. }
\end{aligned}
$$

We are now in a position to prove Theorem 5.1 .

Proof of Theorem 5. 1. It suffices to prove (2. 9). By Lemma 5.4 and 9), we have

$$
\begin{aligned}
& U_{\boldsymbol{t}} \widetilde{f \cdot \lambda}([\boldsymbol{x}, \boldsymbol{p}, j])=\sum_{r=0}^{\infty} U_{t}^{(r)} \widetilde{f \cdot \lambda}([\boldsymbol{x}, \boldsymbol{p}, j]) \\
& =(-1)^{\left[\frac{j}{2}\right]} \lambda^{|\boldsymbol{p}|} \sum_{r=0}^{\infty} \sum_{r_{1}+r_{2}+\cdots+r_{n}=r} \prod_{i=1}^{n} U_{t}^{\left(r_{i}\right)} \widetilde{f \cdot \lambda}\left(\left[x_{\imath}, 0,0\right]\right) \\
& =(-1)^{\left[\frac{j}{2}\right]} \lambda^{|\boldsymbol{p}|} \sum_{r_{1}=0}^{\infty} \sum_{r_{2}=0}^{\infty} \cdots \sum_{r_{n}=0}^{\infty} \prod_{i=1}^{n} U_{t}^{\left(r_{i}\right)} \widetilde{f \cdot \lambda}\left(\left[x_{i}, 0,0\right]\right) \\
& =(-1)^{\left[\frac{j}{2}\right]} \lambda_{\lambda^{|\boldsymbol{p}|}} \prod_{i=1}^{n} \sum_{r_{i}=0}^{\infty} U_{t}^{\left(r_{i}\right)} \widetilde{f \cdot \lambda}\left(\left[x_{i}, 0,0\right]\right) \\
& =\left.(-1)^{\left[\frac{j}{2}\right]} \lambda^{|p|} \prod_{i=1}^{n}\left(U_{t} \widetilde{f \cdot \lambda}\right)\right|_{E}\left(x_{i}\right) \\
& =\left(U_{t} \overparen{f \cdot \lambda)\left.\right|_{E} \cdot \lambda([\boldsymbol{x}, \boldsymbol{p}, j]), \quad f \in \boldsymbol{C}^{*}(E), 0 \leqq \lambda<1,}\right.
\end{aligned}
$$

here $\boldsymbol{x}=\left[x_{1}, x_{2}, \cdots, x_{n}\right]$ and $\boldsymbol{p}=\left[p_{1}, p_{2}, \cdots, p_{n}\right] . \quad$ Q.E.D.

§6. Semi-linear equation. In this section, we shall consider an pplication of Corollary 4.1 to a probabilistic interpretation of the following smi-linear equation:

$$
\frac{\partial u(t, x)}{\partial t}=\frac{1}{2} \Delta u(t, x)+k(x) F(x ; u(t, x))^{19)},
$$

here $k(x)$ is a non-negative bounded continuous function on $R^{d}$ and $F(x ; \xi)$. itisfies the following conditions: there exists a positive constant $K$ such that

$$
\begin{array}{r}
\left|k(x) F(x ; \xi)-k\left(x^{\prime}\right) F\left(x^{\prime} ; \xi^{\prime}\right)\right| \leqq K\left\{\left\|x-x^{\prime}\right\|+\left|\xi-\xi^{\prime}\right|\right\}, \\
x, x^{\prime} \in R^{d}, \xi_{,} \xi^{\prime} \in[0,1],
\end{array}
$$

19) Semi-linear equations of this type are discussed in Kolmogoroff-Petrovsky-Piscounoff 3]. 
where $\left\|x-x^{\prime}\right\|$ denotes the Euclidian distance between $x$ and $x^{\prime}$, and it also holds that

$$
F(x ; 0)=F(x ; 1)=0 \text { and } 0 \leqq F(x ; \xi) \text { for } 0<\xi<1 .
$$

Throughout this section, we shall consider a strong Markov process $Z_{t}$ satisfying Condition 3 (and hence, by Theorem 5.1, a signed branching Markov process with age) whose basic Markov process is a standard Brownian motion on $E=R^{d} \cup\{\infty\}$ which is obtained by the one-point compactification of $R^{d} .{ }^{20)}$

We first consider the special case satisfying Condition $(Q)$ :

(a) Let $q_{n}^{+}(x)$ and $q_{n}^{-}(x)$ be functions given a priori in Condition 3 (through the part (i)). Then $\dot{q}_{0}^{+}(x)=q_{o}^{-}(x)=0$ and there exists an integer $M>0$ such that $q_{n}^{+}(x)=q_{n}^{-}(x)=0$ for $n>M$.

(b) Set

$$
F(x ; \xi)=\sum_{n=1}^{M} \frac{\left\{q_{n}^{+}(x)-q_{n}^{-}(x)\right\}}{k(x)} \xi^{n},
$$

where $k(x)=\sum_{n=1}^{M}\left(q_{n}^{+}(x)+q_{n}^{-}(x)\right) . \quad$ Then

$$
0<F(x ; \xi), \quad x \in R^{d}, \xi \in(0,1),
$$

and also there exists a positive constant $K$ such that

$$
\begin{aligned}
& \left|k(x) F(x ; \xi)-k\left(x^{\prime}\right) F\left(x^{\prime} ; \xi^{\prime}\right)\right| \leqq K\left\{\left\|x-x^{\prime}\right\|+\left|\xi-\xi^{\prime}\right|\right\}, x, x^{\prime} \in R^{d}, \xi, \xi^{\prime} \in[0,1] . \\
& \sum_{n=1}^{M}\left\{q_{n}^{+}(x)-q_{n}^{-}(x)\right\}=0, \quad x \in R^{d} .
\end{aligned}
$$

Condition 3 is called "Condition 3 with $(Q)$ " when $q_{n}^{+}$and $q_{n}^{-}$satisfy Condition $(Q)$.

Lemma 6. 1. Let $Z=\left\{Z_{t}=\left[X_{t}, N_{t}, J_{t}\right], \zeta, \mathscr{B}_{t}, P_{[\boldsymbol{x}, \boldsymbol{p}, j]} ;[\boldsymbol{x}, \boldsymbol{p}, j] \in \widetilde{\boldsymbol{S}}\right\}$ be a signed branching Markov process with age on $\tilde{\boldsymbol{S}}$ satisfying Condition 3 with $(Q)$ and let $U_{t}$ be the semi-group on $\boldsymbol{B}(\widetilde{\boldsymbol{S}})$ induced by $Z^{21)}$. Then there exists a positive number $\delta_{0}$ such that $U_{t} \widetilde{f \cdot 2}([\boldsymbol{x}, \boldsymbol{p}, j])$ exists for any $0 \leqq t<\delta_{0}$, any $f \in \overline{\boldsymbol{C}}^{*}\left(R^{d}\right)$ with $f(\infty)=0^{22)}$ and $[\boldsymbol{x}, \boldsymbol{p}, j] \in \widetilde{\boldsymbol{S}}$.

20) cf. $\S 4$.

21) In Lemma $6.1,(b)$ and $(c)$ in Condition $(Q)$ are not necessary.

22) " $U_{t} \widetilde{f \cdot 2}$ exists" means that $E_{[x, p, j]}\left[|\widetilde{f ; 2}|\left(Z_{t}\right)\right]<\infty$. Also the condition " $f(\infty)=0$ " does not have any influence in the sequel, because $\infty$ is a trap of $X_{t}^{\prime}$ and almost all sample functions of $X_{t}^{\prime}$ with $X_{0}^{\prime}(w) \neq \infty$ do not reach $\infty$ in any finite time interval. 
Proof. First of all, we shall prove the existence of $\delta_{0}>0$ such that $U_{t} \widetilde{1 \cdot 2 \mid}\left([[x, 0,0])=E_{[x, 0,0]}\left[\widetilde{|1 \cdot 2|}\left(Z_{t}\right)\right]\right.$ is finite for any $t<\delta_{0}$ and $x \in R^{d}$, where $1(x) \equiv 1$.

By the same method as in the proof of Lemma 5. 4, we have

$$
U_{t} \widetilde{1 \cdot 2 \mid}([\boldsymbol{x}, \boldsymbol{p}, j])=\sum_{r=0}^{\infty} U_{t}^{(r)} \mid \widetilde{1 \cdot 2 \mid}([\boldsymbol{x}, \boldsymbol{p}, j]), \quad[\boldsymbol{x}, \boldsymbol{p}, j] \in \tilde{\mathbf{S}},
$$

where

$$
U_{t}^{(r)}|\widetilde{1 \cdot 2 \mid}|([\boldsymbol{x}, \boldsymbol{p}, j])=E_{[x, \boldsymbol{p}, j]}\left[\widetilde{1 \cdot \cdot 2 \mid}\left(Z_{t}\right) ; \eta_{r} \leqq t<\eta_{r+1}\right], \quad r \geqq 0 .
$$

Then, by (ii) of Condition 3, Lemma 5.1 and Lemma 4. 1, we have

$$
\begin{gathered}
U_{t}^{o} \mid \widetilde{1 \cdot 2 \mid}([\boldsymbol{x}, \boldsymbol{p}, j])=2^{|\boldsymbol{p}|} U_{t}^{0} \widetilde{[1 \cdot 2 \mid}([\boldsymbol{x}, 0,0])=2^{|\boldsymbol{p}|} \prod_{i=1}^{n} E_{x_{i}}\left[1\left(X_{t}^{\prime}\right)\right]=2^{|\boldsymbol{p}|}, \\
\boldsymbol{x}=\left[x_{1}, x_{2}, \cdots, x_{n}\right], x_{i} \in R^{d}, j \in J,
\end{gathered}
$$

where $E_{x}$ denotes the integral by the probability measure of a standard Brownian motion $X_{t}^{\prime}$. Accordingly, it follows from the strong Markov property of $Z_{t}$ that

$$
\begin{aligned}
U_{t}^{(1)} \mid \widetilde{1 \cdot 2 \mid}([\boldsymbol{x}, 0,0]) & =E_{[x, 0,0]}\left[U_{t-\eta}^{0} \mid \widetilde{1 \cdot 2 \mid}\left(Z_{\eta}\right) ; \eta \leqq t\right] \\
& =\sum_{|p|=0}^{\infty} 2^{|p|} \int_{0}^{t} P_{[x, 0,0]}\left(N_{\eta}=\boldsymbol{p}, \eta \in d s\right) .
\end{aligned}
$$

On the other hand, if we apply (5.4) to $f(t, \cdot)=1$, then we have

$$
\begin{aligned}
& P_{[x, 0,0]}\left(N_{\eta}=\boldsymbol{p}, \eta \in d s\right) \\
&=\sum_{i=1}^{n} \sum_{\substack{p_{l}+\left|\boldsymbol{p}_{i}\right|=|\boldsymbol{p}| \\
l \neq i}} \sum_{m=1}^{M+n-1} \sum_{j=0}^{3} \Psi\left(\left[x_{i}, 0,0\right] ; d s,\left[E^{m}, \boldsymbol{p}_{i}, j\right]\right) \\
& \cdot \prod_{l \neq i} P_{\left[x_{l}, 0,0\right]}\left(N_{s}=p_{l}, s<\eta\right) .
\end{aligned}
$$

Since $Z_{t}$ satisfies Condition 2, (4.7) holds and hence we have

$$
\begin{aligned}
& \sum_{m=1}^{M+n-1} \sum_{j=0}^{3} \Psi\left(\left[x_{i}, 0,0\right] ; d s,\left[E^{m}, \boldsymbol{p}_{i}, j\right]\right) \\
= & P_{\left[x_{i}, 0,0\right]}\left(X_{\eta-} \in R^{d}, \eta \in d s, N_{\eta-}=\left|\boldsymbol{p}_{i}\right|, \sigma_{\left|\boldsymbol{p}_{i}\right|} \leqq s<\sigma_{\left|\boldsymbol{p}_{i}\right|+1}\right) \\
= & E_{x_{i}}\left[e^{-2 \int_{0}^{s} k\left(X_{v}^{\prime}\right) d v} \frac{\left(\int_{0}^{s} k\left(X_{v}^{\prime}\right) d v\right)^{\left|\boldsymbol{p}_{i}\right|}}{\left|\boldsymbol{p}_{i}\right| !} k\left(X_{v}^{\prime}\right) d s\right],
\end{aligned}
$$


and by (3. 11)

$$
P_{\left[x_{l}, 0,0\right]}\left(N_{s}=p_{l}, s<\eta\right)=E_{x}\left[e^{-2 \int_{0}^{s} k\left(X_{v}^{\prime}\right) d v} \frac{\left(\int_{0}^{s} k\left(X_{v}^{\prime}\right) d v\right)^{p_{l}}}{p_{l} !}\right] .
$$

Now let us consider an $n d$-dimensional standard Brownian motion $\left(X_{s}^{(1)}, X_{s}^{(2)}, \cdots, X_{s}^{(n)}\right)$ and denote by $E_{\left(x_{1}, x_{2}, \cdots, x_{n}\right)}$ the integral with respect to the probability measure $P_{\left(x_{1}, x_{2}, \cdots, x_{n}\right)}$ corresponding to $\left(X_{s}^{(1)}, X_{s}^{(2)}, \cdots, X_{s}^{(n)}\right) \cdot{ }^{23)}$ Also we set

$$
\check{g}\left(x_{1}, x_{2}, \cdots, x_{n}\right)=\sum_{i=1}^{n} g\left(x_{i}\right), \quad g \in \boldsymbol{B}\left(R^{d}\right) .
$$

Then it is obtained from (6.7), (6. 8) and (6.9) that

$$
\begin{aligned}
& P_{[\boldsymbol{x}, 0,0]}\left(N_{\eta}=\boldsymbol{p}, \eta \in d s\right) \\
& =\sum_{i=1}^{n} \sum_{l \neq p_{i}} \sum_{\left|\boldsymbol{p}_{i}\right|=|\boldsymbol{p}|} E_{x_{i}}\left[e^{-2 \int_{0}^{s} k\left(X_{v}^{\prime}\right) d v} \frac{\left(\int_{0}^{s} k\left(X_{v}^{\prime}\right) d v\right)^{\left|\boldsymbol{p}_{i}\right|}}{\left|\boldsymbol{p}_{i}\right| !} k\left(X_{s}^{\prime}\right) d s\right] \\
& \cdot \prod_{l \neq i} E_{x_{l}}\left[e^{-2 \int k\left(X_{v}^{\prime}\right) d v} \frac{\left(\int_{0}^{s} k\left(X_{v}^{\prime}\right) d v\right)^{p_{l}}}{p_{l} !}\right] \\
& \text { (6. 10) } \quad=E_{\left(x_{1}, x_{2}, \cdots, x_{n}\right)}\left[e^{-2 \int_{0}^{s} \breve{k}\left(X_{v}^{(1)}, X_{v}^{(2)}, \cdots, X_{v}^{(n)}\right) d v}\right. \\
& \left.\cdot\left\{\sum_{i} \sum_{i=|p|} \prod_{i=1}^{n} \frac{\left(\int_{0}^{s} k\left(X_{v}^{(i)}\right) d v\right)^{p_{i}}}{p_{i} !}\right\} \breve{k}\left(X_{s}^{(1)}, X_{s}^{(2)}, \cdots, X_{s}^{(n)}\right) d s\right] \\
& =E_{\left(x_{1}, x_{2}, \cdots, x_{n}\right)}\left[e^{-2 \int_{0}^{s} k\left(X_{v}^{(1)}, X_{v}^{(2)}, \cdots, X_{v}^{(n)}\right) d v} \frac{1}{|\boldsymbol{p}| !}\left\{\int_{0}^{s} k\left(X_{v}^{(1)}, X_{v}^{(2)}, \cdots, X_{v}^{(n)}\right) d v\right\}^{|\boldsymbol{p}|}\right. \\
& \text { • } \left.\breve{k}\left(X_{s}^{(1)}, X_{s}^{(2)}, \cdots, X_{s}^{(n)}\right) d s\right] \text {. }
\end{aligned}
$$

Applying the above result to the right hand side of (6.6), we have

$$
\begin{aligned}
U_{t}^{(1)} \widetilde{1 \cdot 2 \mid}([\boldsymbol{x}, \mathbf{0}, 0]) & \left.=\int_{0}^{t} E_{\left[x_{1}, x_{2}, \cdots, x_{n}\right]} \check{[k}\left(X_{s}^{(1)}, X_{s}^{(2)}, \cdots, X_{s}^{(n)}\right)\right] d s \\
& =\sum_{i=1}^{n} \int_{0}^{t} E_{x_{i}}\left[k\left(X_{s}^{\prime}\right)\right] d s \\
& \leqq n\|k\| t .
\end{aligned}
$$

23) $X_{s}^{(i)}$ are mutually independent and equivalent standard $d$-dimensional Brownian motions and $x_{i} \in R^{d}, i=1,2, \cdots, n$. 
Now we shall assume that for any $\boldsymbol{x}=\left[x_{1}, x_{2}, \cdots, x_{n}\right]$ and $r \geqq 1$

$$
U_{t}^{(r)} \widetilde{1 \cdot 2 \mid}([\boldsymbol{x}, 0,0]) \leqq n(n+M) \cdots(n+(r-1) M) \frac{(\|k\| t)^{r}}{r !} .
$$

Since we have by the same method as in the proof of Lemma 5.1

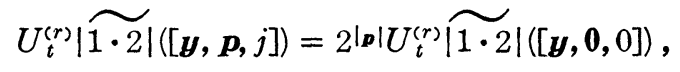

it follows from (6.11) and the strong Markov property of $Z_{t}$ that for $\boldsymbol{x}=\left[x_{1}, x_{2}, \cdots, x_{n}\right]$

$$
\begin{aligned}
& U_{t}^{(r+1)} \widetilde{1 \cdot 2 \mid}([\boldsymbol{x}, \mathbf{0}, 0]) \\
= & \int_{0}^{t} \int_{\tilde{s}} \Psi([\boldsymbol{x}, \mathbf{0}, 0] ; d s,[d \boldsymbol{y}, \boldsymbol{p}, j]) 2^{|\boldsymbol{p}|} U_{t-s}^{(r)} \widetilde{1 \cdot 2 \mid}([\boldsymbol{y}, \mathbf{0}, 0]) \\
\leqq & \int_{0}^{t} \int_{\tilde{s}} \Psi([\boldsymbol{x}, \mathbf{0}, 0] ; d s,[d \boldsymbol{y}, \boldsymbol{p}, j]) 2^{|\boldsymbol{p}|}(n+M)(n+2 M) \cdots(n+r M) \frac{(\|k\|(t-s))^{r}}{r !},
\end{aligned}
$$

because, by the assumption that $q_{n}^{+}(x)=q_{n}^{-}(x)=0$ for $n>M, \Psi([x, 0,0] ; d s$, $\left.\left[E^{m}, \boldsymbol{p}, j\right]\right)=0$ for $m>n+M$. Applying (6.10), the right hand side of the above inequality equals

$$
\begin{aligned}
(n+M)(n+2 M) \cdots(n+r M) \int_{0}^{t} E_{\left(x_{1}, x_{2}, \cdots, x_{n}\right)}\left[\check{k}\left(X_{s}^{(1)}, X_{s}^{(2)}, \cdots, X_{s}^{(n)}\right)\right] & \frac{(\|k\|(t-s))^{r}}{r !} d s \\
& \leqq n(n+M) \cdots(n+r M) \frac{\|k\|^{r+1}}{r !} \int_{0}^{t}(t-s)^{r} d s \\
& =n(n+M) \cdots(n+r M) \frac{(\|k\| t)^{r+1}}{(r+1) !} \cdot
\end{aligned}
$$

Thus (6. 11) holds for any $r \geqq 1$ because it stands for $r=1$.

Now, by (6. 4) and (6.5), we have

$$
\begin{aligned}
U_{t} \mid \widetilde{1 \cdot 2 \mid}([x, 0,0]) & =1+\sum_{r=1}^{\infty} U_{t}^{(r)} \widetilde{1 \cdot 2 \mid}([x, 0,0]) \\
& \leqq 1+\sum_{r=1}^{\infty}(1+M)(1+2 M) \cdots(1+(r-1) M) \frac{(\|k\| t)^{r}}{r !} \\
& \leqq 1+\sum_{r=1}^{\infty}(M\|k\| t)^{r}, \quad x \in E .
\end{aligned}
$$

This shows that $U_{t} \widetilde{1 \cdot 2 \mid}([x, 0,0])$ is finite for any $0 \leqq t<1 / M\|k\|=\delta_{0}$ and $x \in E$. 
Next we prove the finiteness of $U_{t} \mid \widetilde{1 \cdot 2 \mid}([\boldsymbol{x}, \boldsymbol{p}, j])$ for any $t<\delta_{0}$ and $[\boldsymbol{x}, \boldsymbol{p}, j] \in \tilde{\boldsymbol{S}}$. As in the proof of (5.9), we may obtain

$$
\begin{aligned}
U_{t}^{(r)}|\widetilde{1 \cdot 2}|([\boldsymbol{x}, 0,0])= & \underset{r_{1}+r_{2}+\cdots+r_{n}=r}{ } \prod_{i=1}^{n} U_{t}^{\left(r_{i}\right)} \widetilde{|1 \cdot 2|} \mid\left(\left[x_{i}, 0,0\right]\right), \\
& r \geqq 0, x=\left[x_{1}, x_{2}, \cdots, x_{n}\right], t<\delta_{0} .
\end{aligned}
$$

Applying (6.11) to this equation, we have

$$
U_{t}^{(r)} \widetilde{1 \cdot 2 \mid}([x, 0,0]) \leqq \sum_{r_{1}+r_{2}+\cdots+r_{n}=r} \prod_{i=1}^{n}(M\|k\| t)^{r_{i}}, \quad t<\delta_{0} .
$$

Hence it follows from (6.4) and (6.12) that

$$
\begin{aligned}
& U_{t} \widetilde{1 \cdot 2}\left|([\boldsymbol{x}, \boldsymbol{p}, j])=2^{|\boldsymbol{p}|}\right| U_{t}|\widetilde{1 \cdot 2}|([\boldsymbol{x}, 0,0]) \\
& =2^{|\boldsymbol{p}|} \sum_{r=0}^{\infty} U_{t}^{(r)} \mid \widetilde{1 \cdot 2 \mid}([\boldsymbol{x}, \mathbf{0}, 0]) \\
& \leqq 2^{|\boldsymbol{p}|}\left\{\sum_{r=0}^{\infty}(M\|k\| t)^{r}\right\}^{n} \\
& <\infty, \quad 0 \leqq t<\delta_{0},[\boldsymbol{x}, \boldsymbol{p}, j] \in \widetilde{\boldsymbol{S}},
\end{aligned}
$$

as was to be proved.

Q.E.D.

Next we shall consider the following integral equation which turns out to $(6.1)$ :

$$
u(t, x)=\int_{R^{d}}\left(\frac{1}{2 \pi t}\right)^{\frac{d}{2}} e^{-\frac{\|y-x\|^{2}}{2 t}} f(y) d y
$$

$$
\begin{gathered}
+\int_{0}^{t} d s \int_{R^{d}}\left(\frac{1}{2 \pi s}\right)^{\frac{d}{2}} e^{-\frac{\|y-x\|^{2}}{2 s}} k(y) F(y ; u(t-s, y)) d y, \\
t \geqq 0, x \in R^{d},
\end{gathered}
$$

where $k F$ is bounded and satisfies (6.2) for all $x, x^{\prime} \in R^{d}$ and $\xi, \xi^{\prime} \in R^{1}$ and also (6. 3). Further set

$$
\begin{gathered}
u_{0}(t, x)=\int_{R^{d}}\left(\frac{1}{2 \pi t}\right)^{\frac{d}{2}} e^{-\frac{\|y-x\|^{2}}{2 t}} f(y) d y \\
u_{n+1}(t, x)=u_{0}(t, x)+\int_{0}^{t} \int_{R^{d}}\left(\frac{1}{2 \pi s}\right)^{\frac{d}{2}} e^{-\frac{\|y-x\|^{2}}{2 s}} k(y) F\left(y ; u_{n}(t-s, y)\right) d y, \\
t \geqq 0, x \in R^{d}, n \geqq 0 .
\end{gathered}
$$


Then the following result is well known. ${ }^{24)}$

Lemma 6. 2. For a given $f \in \bar{C}^{*}\left(R^{d}\right)^{+}$, the following holds:

(i) Let $u(t, x ; f)$ be the unique solution of (6.13) with initial value $f$. Then we have

$$
0 \leqq u(t, x ; f) \leqq 1, \quad t \geqq 0, x \in R^{d} .
$$

(ii) For any positive constant $T, u_{n}(t, x)$ defined above converges to $u(t, x ; f)$ uniformly in $(t, x) \in[0, T] \times R^{d}$.

Let $Z_{t}$ be a signed branching Markov process with age on $\tilde{\boldsymbol{S}}$ satisfying Condition 3 with $(Q)$ and let $U_{t}$ be the semi-group induced by $Z_{t}$. If we consider the integral equation (6.13), where $k F$ is given by

$$
k(x) F(x ; \xi)=\sum_{n=1}^{M}\left\{q_{n}^{+}(x)-q_{n}^{-}(x)\right\} \xi^{n}, \quad x \in R^{d}, \xi \in R^{1},
$$

then it follows from the uniqueness of the bounded solution of (6.13), Lemma 6. 1 and Theorem 4. 1 that

$$
\left.\left(U_{t} \widetilde{f \cdot 2}\right)\right|_{E}(x)=u(t, x ; f), \quad f \in \bar{C}^{*}\left(R^{d}\right)^{+}, 0 \leqq t<\delta_{0}, x \in R^{d},
$$

where $u(t, x ; f)$ denotes the solution of (6.13) with initial value $f$. On the other hand, by Lemma 6.2 , the solution $\tilde{u}(t, x ; f)$ of the integral equation (6. 13) where $k F$ is replaced by

$$
k(x) F_{1}(x ; \xi)= \begin{cases}\sum_{n=1}^{M}\left\{q_{n}^{+}(x)-q_{n}^{-}(x)\right\} \xi^{n}, & x \in R^{d}, \xi \in[0,1], \\ 0 \quad, & \text { otherwise, }\end{cases}
$$

satisfies ' $0 \leqq \tilde{u}(t, x ; f) \leqq 1$ ' for $f \in \bar{C}^{*}\left(R^{d}\right)^{+}$, because $k F_{1}$ satisfies the condition (6. 3) and (6.2) for all $x, x^{\prime} \in R^{d}$ and $\xi, \xi^{\prime} \in R^{1}$. Since $F(x ; \xi)=F_{1}(x ; \xi)$ for $\xi \in[0,1]$, we have $u(t, x ; f)=\tilde{u}(t, x ; f) \in \overline{\boldsymbol{C}}^{*}\left([0, \infty) \times R^{d}\right)^{+}$. Hence, using Lemma 6. 1 again, we can consider the following:

$$
\begin{aligned}
& U_{t}\left(U_{s} \widetilde{f \cdot 2)}([x, 0,0])=U_{t}\left(\widetilde{\left.\widetilde{f}\left(U_{s}\right)\right|_{E} \cdot 2}\right)([x, 0,0])\right. \\
& =u(t, x ; u(s, \cdot ; f))=u(t+s, x ; f) \text {, } \\
& f \in \bar{C}^{*}\left(R^{d}\right)^{+}, 0 \leqq t, s<\delta_{0}, x \in R^{d} .
\end{aligned}
$$

But, in general, we can not express the left hand side of the above equation

24) cf. Kolmogoroff-Petrovsky-Piscounoff [8], theorems 1, 4 and 6. 
by $\left.\left(U_{t+s} \widetilde{f \cdot 2}\right)\right|_{E}(x)$ because it may happen that $E_{[x, 0,0]}\left[\widetilde{f \cdot 2 \mid}\left(Z_{t}\right)\right]=\infty$. Even so, still we have the following

Theorem 6. 1. Let $Z=\left\{Z_{t}=\left[X_{t}, N_{t}, J_{t}\right], \zeta, \mathscr{B}_{t}, P_{[\boldsymbol{x}, \boldsymbol{p}, j]} ;[\boldsymbol{x}, \boldsymbol{p}, j] \in \widetilde{\boldsymbol{S}}\right\}$ be a signed branching Markov process with age on $\widetilde{\boldsymbol{S}}$ satisfying Condition 3 with $(Q)$ and let $U_{t}$ be the semi-group on $\boldsymbol{B}(\widetilde{\boldsymbol{S}})$ induced by $Z$. Then, for $f \in \overline{\boldsymbol{C}}^{*}\left(R^{d}\right)^{+}$, we can define $\hat{U}_{t} \widetilde{f \cdot 2}([x, 0,0])$ with the following properties:

(i) $\hat{U}_{t} \widetilde{f \cdot 2}([x, 0,0])=\left.\left(U_{t} \widetilde{f \cdot 2}\right)\right|_{E}(x)$ if $U_{t} \widetilde{f \cdot 2}$ exists, $f \in \bar{C}^{*}\left(R^{d}\right)^{+}$.

(ii) $u(t, x)=\hat{U}_{t} \widetilde{f \cdot 2([x, 0,0])}$ is the unique solution of $(6.13)$ with initial value $f \in \bar{C}^{*}\left(R^{d}\right)^{+}$, where $k F$ is given by

$$
k(x) F(x ; \xi)=\sum_{n=1}^{M}\left\{q_{n}^{+}(x)-q_{n}^{-}(x)\right\} \xi^{n}, \quad x \in R^{d}, \xi \in R^{1} .
$$

Proof. According to Lemma 6. 1, there exists $\delta_{0}>0$ such that $U_{t} \widetilde{f \cdot 2}([\boldsymbol{x}, \boldsymbol{p}, j])$ exists for $f \in \overline{\boldsymbol{C}}^{*}\left(R^{d}\right)^{+}, 0 \leqq t<\delta_{0}$ and $[\boldsymbol{x}, \boldsymbol{p}, j] \in \tilde{\boldsymbol{S}}$. Set

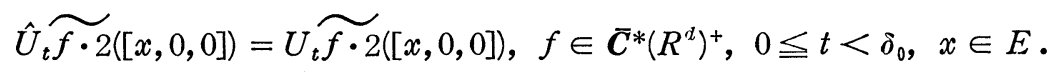

Since $\left.\left(U_{t} \widetilde{f \cdot 2}\right)\right|_{E}(x)=u(t, x ; f) \in \bar{C}^{*}\left(\left[0, \delta_{0}\right) \times R^{d}\right)^{+}$as was mentioned already, $\left.\left(\hat{U}_{t} \widetilde{f \cdot 2}\right)\right|_{E}(x)$ belongs to $\overline{\boldsymbol{C}}^{*}\left(\left[0, \delta_{0}\right) \times R^{d}\right)^{+}$and also $\left.\left(\hat{U}_{t} \widetilde{f \cdot 2}\right)\right|_{E}(\infty)=0$. Using Lemma 6. 1 again, set

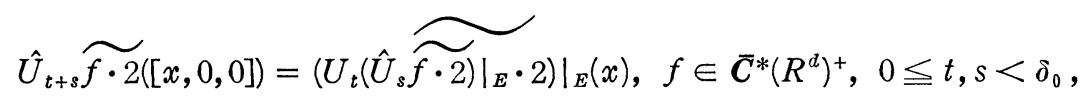

because the right hand side of the above equation is equal to

$$
u\left(t, x ;\left.\left(U_{s} \widetilde{f \cdot 2}\right)\right|_{E}\right)=u(t+s, x ; f),
$$

and hence the right hand side of $(6 \cdot 15)$ depends only on $t+s$ for given $f \in \overline{\boldsymbol{C}}^{*}\left(R^{d}\right)^{+}$and $x \in E$. Repeating this procedure, we can see that $\hat{U}_{t} \widetilde{f \cdot 2}([x, 0,0])$ can be defined for all $t \geqq 0$ and it is the unique solution of (6. 13) with initial value $f \in \overline{\boldsymbol{C}}^{*}\left(R^{d}\right)^{+}$. The property (i) of the theorem is evident by the definition of $\hat{U}_{t}$ and the semi-group property of $U_{t}$. Q.E.D.

In the sequel of this section, we shall use the notation $U_{t}$ instead of

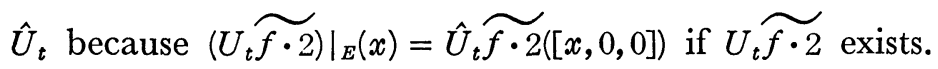


Let $\left\{k_{i}(x),\left(q_{i, n}^{+}(x), q_{i, n}^{-}(x)\right) ; n=1,2, \cdots, M_{i}<\infty\right\}, i=1,2,3, \cdots$, be systems satisfying Condition $(Q)$ and $Z_{t}^{(i)}$ be signed branching Markov processes with age on $\tilde{\boldsymbol{S}}$ satisfying Condition 3 with $(Q)$ for given $\left\{k_{i}(x),\left(q_{i, n}^{+}(x)\right.\right.$, $\left.\left.q_{\bar{i}, n}^{-}(x)\right) ; n=1,2, \cdots, M_{i}\right\}$. Let also $U_{i, t}$ be the semi-group induced by $Z_{t}^{(i)}$ and set

$$
F_{i}(x ; \xi)=\sum_{n=1}^{M_{i}} \frac{\left\{q_{i, n}^{+}(x)-q_{i, n}^{-}(x)\right\}}{k_{i}(x)} \xi^{n}, \quad i=1,2,3, \cdots
$$

According to Theorem 6. 1, if $k_{i} F_{i}$ satisfies

$$
\begin{array}{r}
\left|k_{i}(x) F_{i}(x ; \xi)-k_{i}\left(x^{\prime}\right) F_{i}\left(x^{\prime} ; \xi^{\prime}\right)\right| \leqq K\left\{\left\|x-x^{\prime}\right\|+\left|\xi-\xi^{\prime}\right|\right\}, \\
x, x^{\prime} \in R^{d}, \xi, \xi^{\prime} \in[0,1],
\end{array}
$$

where $K$ is a positive constant independent of $i$, then $u^{(i)}(t, x)=\left.\left(U_{i, t} \widetilde{f \cdot 2}\right)\right|_{E}(x)$ is the solution of the integral equation (6.13) with initial value $f \in \bar{C}^{*}\left(R^{d}\right)^{+}$ where $k F$ is replaced by $k_{i} F_{i}$. Then we have

Theorem 6. 2. Let $\left\{k_{i}(x),\left(q_{i, n}^{+}(x), q_{i, n}^{-}(x)\right) ; n=1,2, \cdots, M_{i}\right\}$ be systems satisfying Condition $(Q)$ and let $k_{i}(x) F_{i}(x ; \xi)$ given in (6.16), $i=1,2,3, \cdots$, satisfy (6. 17). If $k_{i} F_{i}$ converges to $k F$ considered in (6.13) uniformly in $(x, \xi) \in R^{d} \times[0,1]$, then $\left\{u^{(i)}(t, x)=\left.\left(U_{i, t} \widetilde{f \cdot 2}\right)\right|_{E}(x) ; i=1,2,3, \cdots\right\}$ is a uniformly convergent sequence in $(t, x) \in[0, T] \times R^{d}$ for any given $T>0$. Moreover, $u(t, x)=\lim _{i \rightarrow \infty} u^{(i)}(t, x)$ is the unique solution of the integral equation (6.13) with initial value $f \in \bar{C}^{*}\left(R^{d}\right)^{+}$.

Proof. According to (i) of Lemma 6. 2, we may regard $k_{\iota}(x) F_{i}(x ; \xi)=$ $k(x) F(x ; \xi)=0$ for $\xi \notin[0,1]$ so far as we consider the solution of integral equation of type (6.13) with initial value $f \in \bar{C}^{*}\left(R^{d}\right)^{+}$, because $F_{i}$ and $F$ satisfy (6.3) and (6. 17), and hence we may apply Lemma 6.2 in the present case.

Let us set

$$
\begin{gathered}
u_{0}^{(i)}(t, x)=\int_{R^{d}}\left(\frac{1}{2 \pi t}\right)^{\frac{d}{2}} e^{-\frac{\|y-x\|^{2}}{2 t}} f(y) d y, \\
u_{n+1}^{(i)}(t, x)=u_{0}^{(i)}(t, x)+\int_{0}^{t} d s \int_{R^{d}}\left(\frac{1}{2 \pi s}\right)^{\frac{d}{2}} e^{-\frac{\|y-x\|^{2}}{2 s}} k_{i}(y) F_{i}\left(y ; u_{n}^{(i)}(t-s, y)\right) d y, \\
n \geqq 0, \quad i=1,2,3, \cdots .
\end{gathered}
$$

For any given $\varepsilon>0$, we take also $N_{0}$ so large as 
(6. 18)

$$
\left|k_{i}(x) F_{i}(x ; \xi)-k_{j}(x) F_{j}(x ; \xi)\right|<\varepsilon, \quad x \in R^{d}, \xi \in R^{1},{ }^{25)}
$$

holds for any $i, j \geqq N_{0}$. Noting that $u_{0}^{(i)}(t, x)$ is independent of $i$, we can see by (6. 18)

$$
\begin{gathered}
\left|u_{1}^{(i)}(t, x)-u_{1}^{(j)}(t, x)\right| \leqq \int_{0}^{t} d s \int_{R^{d}}\left(\frac{1}{2 \pi s}\right)^{\frac{d}{2}} e^{-\frac{\|y-x\|^{2}}{2 s}} \\
\cdot\left|k_{i}(y) F_{i}\left(y ; u_{0}^{(i)}(y, t-s, y)\right)-k_{j}(y) F_{j}\left(y ; u_{0}^{(j)}(t-s, y)\right)\right| d y \leqq \varepsilon t, \\
x \in R^{d}, i, j \geqq N_{0} .
\end{gathered}
$$

\section{Assume}

$$
\left|u_{n}^{(i)}(t, x)-u_{n}^{(j)}(t, x)\right| \leqq \varepsilon t \sum_{p=0}^{n-1} \frac{(K t)^{p}}{p !},
$$$$
x \in R^{d}, i, j \geqq N_{0},
$$

and it follows from (6.17), (6.18) and (6.19) that

$$
\begin{aligned}
& \quad\left|u_{n+1}^{(i)}(t, x)-u_{n+1}^{(j)}(t, x)\right| \\
& \leqq \int_{0}^{t} d s \int_{R^{d}}\left(\frac{1}{2 \pi s}\right)^{\frac{d}{2}} e^{-\frac{\|y-x\|^{2}}{2 s}}\left\{\left|k_{i}(y) F_{i}\left(y ; u_{n}^{(i)}(t-s, y)\right)-k_{j}(y) F_{j}\left(y ; u_{n}^{(j)}(t-s, y)\right)\right| d y\right. \\
& \leqq \int_{0}^{t} d s \int_{R^{d}}\left(\frac{1}{2 \pi s}\right)^{\frac{d}{2}} e^{-\frac{\|y-x\|^{2}}{2 s}}\left\{\left|k_{i}(y) F_{i}\left(y ; u_{n}^{(i)}(t-s, y)\right)-k_{j}(y) F_{j}\left(y ; u_{n}^{(i)}(t-s, y)\right)\right|\right. \\
& \left.\quad+\left|k_{j}(y) F_{j}\left(y ; u_{n}^{(i)}(t-s, y)\right)-k_{j}(y) F_{j}\left(y ; u_{n}^{(j)}(t-s, y)\right)\right|\right\} d y \\
& \leqq \varepsilon t+\int_{0}^{t} d s \int_{R^{d}}\left(\frac{1}{2 \pi s}\right)^{\frac{d}{2}} e^{-\frac{\|y-x\|^{2}}{2 s}} K\left|u_{n}^{(i)}(t-s, y)-u_{n}^{(j)}(t-s, y)\right| d y \\
& \leqq \varepsilon t+\varepsilon t \sum_{p=1}^{n} \frac{(K t)^{p}}{p !} \\
& =\varepsilon t \sum_{p=0}^{n} \frac{(K t)^{p}}{p !}, \quad x \in R^{d}, i, j \geqq N_{0} .{ }^{26)}
\end{aligned}
$$

Therefore, by induction, (6. 19) holds for any $n \geqq 0$. Then (ii) of Lemma 6. 2 shows that

$$
\left|u^{(i)}(t, x)-u^{(j)}(t, x)\right| \leqq \varepsilon t e^{K t}, \quad i, j \geqq N_{0} .
$$

Since $\varepsilon>0$ is arbitrary, the above inequality proves the first half of the theorem.

25) We regard $k_{i}(x) F_{i}(x ; \xi)=k(x) F(x ; \xi)=0$ for $\xi \notin[0,1]$.

26) Assume $K \geqq 1$, if necessary. 
On the other hand, $u^{(\imath)}(t, x)$ is the solution of

$$
u^{(i)}(t, x)=u_{0}^{(i)}(t, x)+\int_{0}^{t} d s \int_{R^{d}}\left(\frac{1}{2 \pi s}\right)^{\frac{d}{2}} e^{-\frac{\|y-x \mid\|^{2}}{2 s}} k_{i}(y) F_{i}\left(y ; u^{(i)}(t-s, y)\right) d y .
$$

Letting $i$ tend to infinity in the above equation, we can see that $u(t, x)=$ $\lim _{i \rightarrow \infty} u^{(i)}(t, x)$ is the unique solution of (6.13) with initial value $f \in \bar{C}^{*}\left(R^{d}\right)^{+}$.

Q.E.D.

Transforming (6.13) into the corresponding differential equation, we have

Corollary 6. 1. Let $k_{i}(x) F_{i}(x, u), u^{(i)}(t, x)$ and $k(x) F(x, u)$ be functions as in Theorem 6.3. Then $u(t, x)=\lim _{i=\infty} u^{(i)}(t, u)$ is the bounded solution of the parabolic equation

$$
\frac{\partial u(t, x)}{\partial t}=\frac{1}{2} \Delta u(t, x)+k(x) F(x ; u(t, x)),
$$

with initial value $f \in \overline{\boldsymbol{C}}^{*}\left(R^{d}\right)^{+}$.

In the following corollary, we consider the case where $k(x)$ is a positive constant and $F(x ; \xi)$ is a function of $\xi$ alone.

Corollary 6. 2. Let $F(\xi)$ be a function which is continuously differentiable on $[0,1]$ and $F^{\prime}(0)>0$. Let also $F(\xi)$ satisfies the condition:

$$
F(0)=F(1)=0 \text { and } 0<F(\xi) \text { for } 0<\xi<1 \text {. }
$$

Then the unique solution $u(t, x ; f)$ of the parabolic equation

$$
\frac{\partial u(t, x)}{\partial t}=\frac{1}{2} \Delta u(t, x)+F(u),
$$

with initial value $f \in C^{*}\left(R^{d}\right)^{+}$is expressed as the limit of $u^{(i)}(t, x)$ of the type which appeared in Theorem 6. 2.

Proof. Since $F^{\prime}(\xi)$ is continuous on $[0,1]$, there exists a sequence of polynomials $g_{i}^{\prime}(\xi)$ converging to $F^{\prime}(\xi)$ uniformly on $[0,1]$. Set

$$
G_{i}(\xi)=\int_{0}^{\xi} g_{i}^{\prime}(s) d s+c_{i} \xi, \quad i=1,2,3, \cdots,
$$

where $c_{i}$ is chosen so that $G_{i}(1)=0$. Then $c_{i}$ tends to zero as $i$ increases, because $F(1)=0$ and $g_{i}^{\prime}(\xi)$ converge to $F^{\prime}(\xi)$ uniformly on $[0,1]$. Hence the polynomials $G_{i}(\xi)$ converge to $F(\xi)$ uniformly on $[0,1]$ and $G_{i}^{\prime}(\xi)$ is uni- 
formly bounded. Moreover, $\xi_{i}=\inf \left\{\xi>0 ; G_{i}(\xi)=0\right\}$ tends to 1 as $i$ increases because $F^{\prime}(0)>0$ and $F(\xi)>0$ for $0<\xi<1$. Expressing $G_{i}(\xi)$ in the following form:

$$
\left.G_{i}(\xi)=\sum_{n=1}^{M_{i}}\left(q_{i, n}^{+}-q_{\bar{i}, n}\right) \xi^{n}, \quad i=1,2,3, \cdots,{ }^{27}\right)
$$

where $q_{i, n}^{+}$and $q_{\bar{i}, n}$ are non-negative constants such that $q_{i, n}^{+} q_{\bar{i}, n}=0$ and $\sum_{n=1}^{M_{i}}\left(q_{i, n}^{+}-q_{\bar{i}, n}\right)=0$, we set

$$
\begin{aligned}
& k_{i}=\sum_{n=1}^{M_{1}}\left(q_{i, n}^{+}+q_{\bar{i}, n}^{-}\right), \\
& F_{i}(\xi)=\frac{1}{k_{i}} \sum_{n=1}^{M_{i}}\left(q_{i, n}^{+}-q_{\bar{i}, n}\right) \xi^{n},
\end{aligned}
$$

Since $k_{i} F_{i}=G_{i}, k_{i} F_{i}(\xi)$ converges to $F(\xi)$ uniformly on $[0,1]$, and there exists a positive constant $K$ such that

$$
\left|k_{i} F_{i}(\xi)-k_{i} F_{i}\left(\xi^{\prime}\right)\right| \leqq K\left|\xi-\xi^{\prime}\right|, \quad \xi, \xi^{\prime} \in[0,1], \quad i=1,2,3, \cdots .
$$

Also it holds that

$$
\begin{aligned}
& 0<F_{i}(\xi), \quad 0<\xi<\xi_{i}, \\
& k_{i} F_{i}(0)=k_{i} F_{i}\left(\xi_{i}\right)=0,
\end{aligned} \quad i=1,2,3, \cdots .
$$

Now let $Z^{(i)}$ be signed branching Markov process with age on $\tilde{\boldsymbol{S}}$ satisfying Condition 3 with $(Q)$ for $\left\{\left(q_{i, n}^{+}, q_{\bar{i}, n}^{-}\right) ; n=1,2, \cdots, M_{i}\right\}$ given above where the condition $0<F(x ; \xi), 0<\xi<1$, is replaced by $0<F_{i}(\xi)$ for $0<\xi<\xi_{i}$, and let $U_{i, t}$ be the semi-group induced by $Z^{(i)}$. Then, by Theorem 6. 1, $u^{(i)}(t, x)=\left.\left(U_{i, t} \widetilde{f \cdot 2}\right)\right|_{E}(x)$ is the solution of the integal equation of (6.13), where $k F$ is replaced by $k_{i} F_{i}$, with initial value $f \in C^{*}\left(R^{d}\right)^{+}$ whose norm $\|f\|$ is less than $\xi_{i}$. Moreover, it holds that

$$
0 \leqq u^{(i)}(t, x) \leqq \xi_{i} \leqq 1 .^{28)}
$$

Since $k_{i} F_{i}(\xi)$ converge to $F(\xi)$ uniformly on $[0,1]$, we can see, as in the proof of the convergence of $u^{(i)}(t, x)$ in Theorem 6.2, that $u^{(i)}(t, x)$ converges to the solution of (6.21) with initial value $f \in C^{*}\left(R^{d}\right)^{+}$, where $\|f\| \leqq$ inf $\left\{\xi_{i} ; i=1,2,3, \cdots\right\}$, because the integral equation of type (6.13) is equiva-

27) Since $G_{i}(0)=0$, the constant term of $G_{i}(\xi)$ is zero.

28) $k_{i} F_{i}(0)=k_{i} F\left(\xi_{i}\right)=0$ and $k_{i} F(\xi)>0$ for $0<\xi<\xi_{i}$. So we may consider $\xi_{i}$ instead of 1 in Theorem 6.1. 
lent to (6. 21) in the present case. On the other hand, $\xi_{i}$ tends to one as $i$ increases, and hence the same assertion holds for any $f \in C^{*}\left(R^{d}\right)^{+}$.

$$
\text { Q.E.D. }
$$

\section{§. Construction of signed branching Markov processes with} age (I). (Non-branching part.)

In this and in the next section, we shall construct the process discussed in the previous sections. Although such a process can be constructed by continuation of sample paths, ${ }^{29)}$ we shall here construct them by an analytic method originated by J.E. Moyal [10].

In this section, we shall deal with a process corresponding to a nonbranching part. For this purpose, we construct a process which is able to describe the creation of mass, i.e. using the process, we can interpret probabilistically the parabolic equation:

$$
\frac{\partial u(t, x)}{\partial t}=\frac{1}{2} \Delta u(t, x)+k(x) u(t, x), \quad x \in R^{d},
$$

where $k(x)$ is a bounded continuous continuous function on $R^{d}$.

First, we shall state some known results which are useful for the construction of our processes. Let $S$ be a locally compact Hausdorff space satisfying the second axiom of countability and let $\chi_{0}(t, x, \cdot)$ and $\Psi(x ; t, \cdot)$ be measures on $\mathscr{B}(S)$ for fixed $x \in S$ and $t \geqq 0$. Let also $\chi_{0}(t, \cdot, B)$ and $\Psi(\cdot ; t, B)$ be Borel measurable functions for fixed $t$ and $B \in(S)$. Let the pair of $\chi_{0}$ and $\Psi$ also satisfy the following conditions:

$$
\begin{aligned}
& \chi_{0}(t+s, x, B)=\int_{S} \chi_{0}(t, x, d y) \chi_{0}(s, y, B), \\
& \lim _{t \rightarrow \infty} \Psi(x ; t, S)=1-\lim _{t \rightarrow \infty} \chi_{0}(t, x, B), \\
& \Psi(x ; t+s, B)=\Psi(x ; t, B)+\int_{S} \chi_{0}(t, x, d y) \Psi(y ; s, B), \\
& \Psi(x ; t, S) \text { is continuous in } t, \\
& \quad x \in S, B \in \mathscr{B}(S), t, s \geqq 0 .
\end{aligned}
$$

Then it is said that $\chi_{0}$ and $\Psi$ satisfy the $\chi_{0} \Psi$-condition. ${ }^{30}$ When $\chi_{0}$ and $\Psi$

29) cf. M. Nagasawa [13].

30) Moyal's $\chi_{0} \Psi$-condition is stated for non stationary Markov processes. The condition stated here is the one for the stationary case and is strengthened in the part of (7.5). (cf. Moyal [10].) 
satisfy the $\chi_{0} \Psi$-condition, by (7. 4), $\Psi(x ; t, B)$ is nomotone non-decreasing in $t$. Let $\Psi(x ; d t, B)$ be the measure induced by $\Psi(x ; t, B)$ for fixed $x$ and $B$. Wè define $\Psi_{r}$ and $\chi_{r}$ by

$$
\Psi_{1}(x ; d t, B)=\Psi(x ; d t, B)
$$

$$
\begin{array}{ll}
\Psi_{r+1}(x ; d t, B)=\int_{0}^{t} \int_{S} \Psi_{r}(x ; d s, d z) \Psi(z ; d(t-s), B), & r \geqq 0, \\
\Psi_{r}(x ; t, B)=\int_{0}^{t} \Psi_{r}(x ; d s, B), & r \geqq 1, \\
\chi_{r}(t, x, B)=\int_{0}^{t} \int_{S} \Psi_{r}(x ; d s, d z) \chi_{0}(t-s, z, B), & r \geqq 1 .
\end{array}
$$

Then we have the following

Lemma 7. 131) (J.E. Moyal) If the $\chi_{0} \Psi$-condition is satisfied, then it holds that: (i)

$$
\Psi_{r+r^{\prime}}(x ; d t, B)=\int_{0}^{t} \int_{S} \Psi_{r}(x ; d s, d y) \Psi_{r^{\prime}}(y ; d(t-s), B), \quad r, r^{\prime} \geqq 1,
$$

(7. 9) $\quad \chi_{r+r^{\prime}}(t, x, B)=\int_{0}^{t} \int_{S} \Psi_{r}(x ; d s, d y) \chi_{r^{\prime}}(t-s, y, B), \quad r \geqq 1, r^{\prime} \geqq 0$,

(7. 10) $\chi_{r}(t+s, x, B)=\sum_{r^{\prime}=0}^{r} \int_{S} \chi_{r^{\prime}}(t, x, d y) \chi_{r-r^{\prime}}(s, y, B), \quad r \geqq 0$,

(7. 11) $\sum_{r=0}^{\infty} \chi_{r}(t, x, S)=1-\lim _{r \rightarrow \infty} \Psi_{r}(x ; t, S), \quad x \in S, B \in \mathscr{B}(S), t, s \geqq 0$.

(ii) The function $\chi$ defined by

$$
\chi(t, x, B)=\sum_{r=0}^{\infty} \chi_{r}(t, x, B), \quad x \in S, B \in \mathscr{B}(S), t \geqq 0,
$$

\section{satisfies}

$$
\chi(t+s, x, B)=\int_{S} \chi(t, x, d y) \chi(s, y, B)
$$

and

$$
\chi(t, x, B)=\chi_{0}(t, x, B)+\int_{0}^{t} \int_{S} \Psi(x ; d s, y) \chi(t-s, y, B)
$$

31) cf. J.E. Moyal [9], theorems in $\$ \S 2-8$. 
(iii) For given $\chi_{0}$ and $\Psi, \chi$ is the minimal non-negative solution of (7. 14), and if

$$
\lim _{r \rightarrow \infty} \Psi_{r}(x ; t, S)=0
$$

holds, then $\chi$ is the unique solution of (7.14).

Now let us set

$$
\begin{array}{ll}
T_{t}^{(r)} f(x)=\int_{S} \chi_{r}(t, x, d y) f(y), & r \geqq 0, \\
T_{t} f(x)=\int_{S} \chi(t, x, d y) f(y), &
\end{array}
$$

According to (7.2) and (7. 13), there exist two Markov processes (but we do not assume the right continuity of sample paths here) $X_{t}^{0}$ and $X_{t}$ whose semi-groups are given by $T_{t}^{(0)}$ and $T_{t}$ respectively. When we consider that there exists a Markov time $\tau$ of $X_{t}$ and $X_{t}^{0}$ is the process obtained by the killing of $X_{t}$ at the time $\tau$, it is expected that $\Psi(x ; d t, B)$ denotes $P_{x}\left(\tau \in d s, X_{\tau} \in B\right)$ under certain conditions, where $P_{x}$ denotes the probability measure of $X_{t}$. About this, we quote from Sirao [17] the following

Lemma 7. 2.32) Let $\Psi_{r}, \chi_{r}$ and $\chi$ be the functions defined by (7.6), (7. 7) and (7. 12). Let them also satisfy the following conditions: (a) $T_{t}^{(0)}$ given in (7. 16) is strongly continuous on $C_{0}(S)$. (b) $T_{t}^{(r)}$ given in (7.16) maps $C_{0}(S)$ into itself and also we have

$$
\lim _{t \rightarrow 0}\left\|T_{t}^{(r)} f\right\|=0, \quad r \geqq 1, f \in C_{0}(S) .
$$

Then it holds that (i) there exists a strong Markov process $X=\left\{X_{t}, \zeta, \mathscr{B}_{t}, P_{x} ; x \in S\right\}$ corresponding to the semi-group $T_{t}$ given in (7.16) whose sample paths are right continuous and quasi left continuous, ${ }^{33)}$ (ii) there exists a $\left(\mathscr{B}_{t^{-}}\right)$Markov time $\tau$ of $X_{t}$ such that there exists a strong Markov process $X^{0}=\left\{X_{t}^{0}, \tau, \mathscr{B}_{t}^{0}, P_{x}^{0} ; x \in S\right\}$ corresponding to the semi-group $T_{t}^{(0)}$ and $X^{0}$ is the killed process of $X$ at the time $\tau$, and (iii) setting

32) cf. [17], Theorem 1.

33) A right continuous strong Markov process $X_{t}$ on $\mathscr{X}$ is said to be quasi left continuous if, for any monotone non-decreasing sequence $\left\{\tau_{n} ; n \geqq 0\right\}$ of Markov times,

$$
P_{x}\left(\lim _{n \rightarrow \infty} X_{\tau_{n}}=X_{\tau}, \tau<\varsigma\right)=P_{x}(\tau<\varsigma), \quad x \in \mathscr{X},
$$

holds, where $\tau=\lim _{n \rightarrow \infty} \tau_{n}$ and $\varsigma$ denotes the terminal time of $X_{t}$. 


$$
\left.\tau_{0}=\tau, \tau_{1}=\tau, \tau_{r+1}=\tau_{r}+\theta \tau_{r} \tau, \quad r \geqq 1,34\right)
$$

we have

$$
\begin{aligned}
& P_{x}\left(X_{t} \in B, \tau_{r} \leqq t<\tau_{r+1}\right)=\chi_{r}(t, x, B) \\
& P_{x}\left(X_{\tau_{r}} \in B, \tau_{r} \in d t\right)=\Psi_{r}(x ; d t, B), \\
& \quad x \in S, B \in \mathscr{B}(S), t \geqq 0, r \geqq 0 .
\end{aligned}
$$

Now let us apply the above lemmas for our case. In the sequel of this section, let $E$ be a locally compact Hausdorff space ${ }^{35}$ ) satisfying the second axiom of countability and $X^{\prime}=\left\{X_{t}^{\prime}, \mathscr{B}_{t}^{\prime}, P_{x} ; x \in E\right\}$ be a conservative Feller process. Then the semi-group $H_{t}$ induced by $X_{t}^{\prime}$ is strongly continuous on $C_{0}(E) .{ }^{36)}$ As in $\S 2$, we shall consider the topological sum $S=\bigcup_{p=0}^{\infty} E \times\{p\}$ $=E \times N$, where $N=\{0,1,2, \cdots\}$. Then $S \cup\{\delta\}, \delta$ being an isolated point, is a locally compact Hausdorff space satisfying the second axiom of countability. A point of $S$ and a Borel sub-set of $S$ are denoted by $[x, p]$ and $[A, p]$ respectively, where $A \in \mathscr{B}(E)$.

Let $k(x)$ be a bounded continuous function on $E$ and let $k(x)=k^{+}(x)-k^{-}(x)$ where $k^{+}(x)=\max (k(x), 0)$ and $k^{-}(x)=\max (-k(x), 0)$. Then

$$
\begin{aligned}
& \varphi_{t}(w)=\int_{0}^{t}\left|k\left(X_{s}^{\prime}(w)\right)\right| d s, \\
& \varphi_{t}^{+}(w)=\int_{0}^{t} k^{+}\left(X_{s}^{\prime}(w)\right) d s, \\
& \varphi_{t}^{-}(w)=\int_{0}^{t} k^{-}\left(X_{s}^{\prime}(w)\right) d s,
\end{aligned}
$$

are non-negative additive functionals of $X^{\prime}$ and hence we can consider the $\exp \left(-\varphi_{t}\right)$ sub-process of $X^{\prime}$, which will be denoted by $X^{0}=\left\{X_{t}^{0}, \sigma, \mathscr{B}_{t}^{0}, P_{x}^{0} ; x \in E\right\}$. Then it trivially holds that

$$
\begin{gathered}
P_{x}^{0}\left(X_{t}^{0} \in B\right)=P_{x}^{0}\left(X_{t}^{0} \in B, t<\sigma\right)=E_{x}\left[e^{-\varphi_{t}} ; X_{t}^{\prime} \in B\right], \\
x \in E, B \in \mathscr{B}(E),
\end{gathered}
$$

31) $\tau_{r}$ 's are Markov times. cf. Ito-McKean [7], p. 87.

35) In this section, we do not assume that $E$ is a compact space, because we consider the equation of type (7.1) with initial value $f \in C(E)$ and the assumption of non-compactness does not cause any difficulty in the discussions of this section.

36) When we consider $H_{t}$ on $C_{0}(E), H_{t}$ may be regarded as the semi-group on $C(E \cup\{\infty\})$ where $E \cup\{\infty\}$ denotes the one-point compactification of $E$. Then it is known that the convergence of $H_{t} f(x)$ to $f(x)$ at any $x \in E$ implies the strong convergence of $H_{t} f$ to $f$, i.e. $\left\|H_{t} f-f\right\| \rightarrow 0$ as $t \rightarrow 0$. (For instance, cf. Dynkin [2], Theorem 5.) 
where $E_{x}$ denotes the integral by $P_{x}$.

Now let us set

$$
\chi_{0}(t,[x, p],[B, q])= \begin{cases}\delta_{p q} P_{x}^{0}\left(X_{t}^{0} \in B\right), & x \in E, \\ 0 & , \text { if }[x, p]=\delta \text { and } \delta \notin[B, q], \\ 1 & , \text { if }[x, p]=\delta \text { and } \delta \in[B, q],\end{cases}
$$

and

$$
\text { (7. 22) } \Psi([x, p] ; d t,[B, q])=\left\{\begin{array}{r}
E_{x}\left[e^{-\varphi_{t}} \frac{d \varphi_{t}^{+}}{d t} d t ; X_{t}^{\prime} \in B\right], \text { if } x \in E \text { and } q=p+1, \\
E_{x}\left[e^{-\varphi_{t}} \frac{d \varphi_{t}^{-}}{d t} d t\right], \text { if } x \in E \text { and } \delta \in[B, q], \\
0 \quad, \text { otherwise, }{ }^{37)} \\
{[x, p] \in S,[B, q] \in \mathscr{B}(S \cup\{\delta\}),}
\end{array}\right.
$$

where $\delta_{p q}$ denotes Kroncecker's delta. Then $\chi_{0}$ is a measure on $\mathscr{B}(S \cup\{\delta\})$ with parameters $t$ and $[x, p] \in S \cup\{\delta\}$ and $\Psi$ is a measure on $\mathscr{B}([0, \infty) \times S \cup\{\delta\})$ with parameter $[x, p] \in S \cup\{\delta\}$. Moreover $\chi_{0}(t,[x, p],[B, p+q])$ and $\Psi([x, p]$; $d t,[B, p+q+1])$ are independent of $p$ and vanish for $q \neq 0$. Let also set

$$
\begin{aligned}
\Psi([x, p] ; t,[B, q]) & =\int_{0}^{t} \Psi([x, p] ; d s,[B, q]), \\
{[x, p] } & \in S \cup\{\delta\}, t \geqq 0,[B, q] \in \mathscr{B}(S \cup\{\delta\}] .
\end{aligned}
$$

Then we have

Lemma 7. 3. Let $\chi_{0}([x, p], t, \cdot)$ and $\Psi([x, p] ; t, \cdot)$ be measures given in (7. 21) and (7.23) respectively. Then they satisfy the Moyal's $\chi_{0} \Psi$-condition.

Proof. By the definition of $\chi_{0}$ and $\Psi, \chi_{0}(t, \delta,\{\delta\})=1$ and $\Psi(\delta ; t, S)=0$ for any $t \geqq 0$. So it suffices to show that the conditions (7.2) - (7.5) hold for $[x, p] \in S$.

Since $X_{t}^{0}$ is a Markov process and $\chi_{0}([x, p], t,[\cdot, p])$ corresponds to the transition function of $X_{t}^{0},(7.2)$ holds evidently.

Combining (7.22) and (7.23), we can see that

$$
\Psi([x, p] ; t, S \cup\{\delta\})=E_{x}\left[\int_{0}^{t} e^{-\varphi_{s}} d\left(\varphi_{s}^{+}+\varphi_{s}^{-}\right)\right]
$$

37) $\frac{d \varphi_{t}}{d t}, \frac{d \varphi_{t}^{+}}{d t}$ and $\frac{d \varphi_{t}^{-}}{d t}$ denote the derivatives of $\varphi_{t}, \varphi_{t}^{+}$and $\varphi_{\bar{t}}$ in the sense of Radon-Nikodym respectively. 


$$
\begin{aligned}
& =E_{x}\left[\int_{0}^{t}\left(-d e^{-\varphi_{s}}\right)\right] \\
& =E_{x}\left[1-e^{-\varphi_{t}}\right] \\
& =1-\chi_{0}(t,[x, p], S \cup\{\delta\}), \quad[x, p] \in S,
\end{aligned}
$$

which proves (7.3).

Now set $B^{+}=B \cap\{x ; k(x) \geqq 0\}$ and $B^{-}=B \cap\{x ; k(x)<0\}$ for any $B \in \mathscr{B}(E)$. Then we have

$$
\begin{aligned}
& \Psi([x, p] ; t+s,[B, p+1]) \\
= & E_{x}\left[\int_{0}^{t+s} e^{-\varphi_{v}} I_{B}\left(\left(X_{v}^{\prime}\right) d \varphi_{v}^{+}\right]\right. \\
= & E_{x}\left[\int_{0}^{t} e^{-\varphi_{v}} I_{B}\left(X_{v}^{\prime}\right) d \varphi_{v}^{+}\right]+E_{x}\left[e^{-\varphi_{t}} E_{X_{t}^{\prime}}\left[\int_{0}^{s} e^{-\varphi_{v}} I_{B}\left(X_{v}^{\prime}\right) d \varphi_{v}^{+}\right]\right] \\
= & \Psi([x, p] ; t,[B, p+1])+\int_{E} \chi_{0}(t,[x, p],[d y, p]) \Psi([y, p] ; s,[B, p+1]) \\
= & \Psi([x, p] ; t,[B, p+1])+\int_{S \cup\{\delta\}} \chi_{0}(t,[x, p],[d y, q]) \Psi([y, q] ; s,[B, p+1]), \\
\qquad & \quad[x, p] \in S, t, s \geqq 0, B \in \mathscr{B}(E),
\end{aligned}
$$

where $I_{B}$ denotes the indicator function of $B$. Similarly we get

$$
\begin{aligned}
& \Psi([x, p] ; t+s,\{\delta\}) \\
= & \Psi([x, p] ; t,\{\delta\})+\int_{S \cup\{\delta\}} \chi_{0}(t,[x, p],[d y, q]) \Psi([y, q] ; s,\{\delta\}) .
\end{aligned}
$$

The above two equations prove (7. 4), because $\chi_{0}(t,[x, p],[B, q])=\Psi([x, p]$; $t,[B, q+1])=0$ for $p \neq q, B \in \mathscr{B}(E)$.

Since (7.5) is evident by the definition of $\Psi$, we have proved the lemma.

Q.E.D.

Now let us set

$$
\begin{aligned}
& \Psi_{1}([x, p] ; d t,[B, q])=\Psi([x, p] ; d t,[B, q]), \\
& \Psi_{r+1}([x, p] ; d t,[B, q])=\int_{0}^{t} \int_{S \cup\{\delta\}} \Psi_{r}\left([x, p] ; d s,\left[d y, p^{\prime}\right]\right) \Psi\left(\left[y, p^{\prime}\right] ; d(t-s),[B, q]\right), \\
& \Psi_{r}([x, p] ; t,[B, q])=\int_{0}^{t} \Psi_{r}([x, p] ; d s,[B, q]),
\end{aligned}
$$

$$
\chi_{r}(t,[x, p],[B, q])=\int_{0}^{t} \int_{S \cup\{\delta\}} \Psi_{r}\left([x, p] ; d s,\left[d y, p^{\prime}\right]\right) \chi_{0}\left(t-s,\left[y, p^{\prime}\right],[B, q]\right),
$$




$$
\begin{aligned}
\chi(t,[x, p],[B, q]) & =\sum_{r=0}^{\infty} \chi_{r}(t,[x, p],[B, q]), \\
r & \geqq 1,[x, p] \in S \cup\{\delta\},[B, q] \in \mathscr{B}(S \cup\{\delta\}], t \geqq 0 .
\end{aligned}
$$

Then we may apply Lemma 7.1 for our $\chi_{r}, \Psi_{r}$ and $\chi_{\text {. }}$

Lemma 7. 4. Let $\Psi_{r}$ be defined in (7. 24). Then we have

$$
\lim _{r \rightarrow \infty} \Psi_{r}([x, p] ; t, S \cup\{\delta\})=0,
$$

for any $t \geqq 0$.

Proof. When $[x, p]=\delta, \quad(7.25)$ is evident. Let $[x, p] \in S$ and let $B \in \mathscr{B}(E)$. First we shall prove

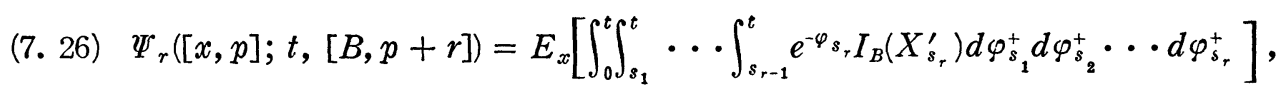

$$
r \geqq 1 \text {. }
$$

By the definition of $\Psi_{1}$, (7. 26) holds for $r=1$. Assume that (7. 26) holds for $r$. Then we can obtain from Lemma 7.1 and the strong Markov property of $X^{\prime}$ that

$$
\begin{aligned}
& \Psi_{r+1}([x, p] ; t,[B, p+r+1]) \\
= & \int_{0}^{t} \int_{0}^{v} \int_{S \cup\{\delta\}} \Psi_{1}([x, p] ; d s,\lceil d y, q]) \Psi_{r}([y, q] ; d(v-s),[B, p+r+1]) \\
= & \int_{0}^{t} \int_{E} \Psi_{1}([x, p] ; d s,[d y, p+1]) \Psi_{r}([y, p+1] ; t-s,[B, p+r+1]) \\
= & E_{x}\left[\int_{0}^{t} e^{-\varphi_{s}} d \varphi_{s}^{+} E_{X_{s}^{\prime}}\left[\int_{0}^{t-s} \int_{s_{1}}^{t-s} \cdots \int_{s_{r-1}}^{t-s} e^{-\varphi_{s_{r}}} I_{B}\left(X_{s_{r}}^{\prime}\right) d \varphi_{s_{1}}^{+} d \varphi_{s_{2}}^{+} \cdots d \varphi_{s_{r}}^{+}\right]\right] \\
= & E_{x}\left[\int_{0}^{t} \int_{v_{1}}^{t} \cdots \int_{v_{r}}^{t} e^{-\varphi}{ }_{v_{r+1}} I_{B}\left(X_{v_{r+1}}^{\prime}\right) d \varphi_{v_{1}}^{+} d \varphi_{v_{2}}^{+} \cdots d \varphi_{v_{r+1}}^{+}\right],
\end{aligned}
$$

which shows the validity of $(7.26)$ for $r+1$. So we can see inductively the validity of (7.26) for any $r \geqq 0$.

Similarly we get

$$
\begin{aligned}
& \Psi_{r}([x, p] ; t,\{\delta\}) \\
= & E_{x}\left[\int_{0}^{t} \int_{s_{1}}^{t} \cdots \int_{s_{r-1}}^{t} e^{-\varphi_{s r}} d \varphi_{s_{1}}^{+} d \varphi_{s_{2}}^{+} \cdots d \varphi_{s_{r-1}}^{+} d \varphi_{s_{r}}^{-}\right], \quad r \geqq 0 .
\end{aligned}
$$

Combining (7. 26) with the above equation, we have 


$$
\begin{aligned}
& \Psi_{r}([x, p] ; t, S \cup\{\delta\}) \\
& =E_{x}\left[\int_{0}^{t} \int_{s_{1}}^{t} \cdots \int_{s_{r-1}}^{t} e^{-\varphi_{s_{r}}} d \varphi_{s_{1}}^{+} d \varphi_{s_{2}}^{+} \cdots d \varphi_{s_{r-1}}^{+} d \varphi_{s_{r}}\right], \quad r \geqq 1 .
\end{aligned}
$$

Since $k(x)$ is bounded on $E$, it follows from the definitions of $\varphi_{s}, \varphi_{s}^{+}, \varphi_{s}^{-}$ and the above equation that

$$
\Psi_{r}([x, p] ; t, S \cup\{\delta\}) \leqq \frac{(\|k\| t)^{r}}{r !}, \quad r \geqq 1,
$$

which proves the lemma.

Q.E.D.

Here we note that

$$
\chi(t,[x, p], S \cup\{\delta\})=1, \quad[x, p] \in S \cup\{\delta\},
$$

which follows from (7.11), (7.12) and (7.25).

Let us now consider the function space

$$
\boldsymbol{C}_{0}(S \cup\{\delta\})=\left\{f ; f(\delta)=0,\left.f\right|_{S} \in \boldsymbol{C}_{0}(S)\right\},
$$

where $\left.f\right|_{S}$ denotes the restricted function of $f$ on $S$. Let also $V_{t}$ be the operator defined by

$$
\begin{aligned}
V_{t} f([x, p]) & =\int_{S \cup\{\delta\}} \chi(t,[x, p],[d y, q]) f([y, q]), \\
& f \in C_{0}(S \cup\{\delta\}),[x, p] \in S \cup\{\delta\}, t \geqq 0 .
\end{aligned}
$$

Then we have

Theorem 7.1. Let $H_{t}$ be the semi-group on $C_{0}(E)$ induced by the Feller process $X_{t}^{\prime}$. Then $V_{t}$ mentioned above is a strongly continuous and non-negative. contraction semi-group on $\boldsymbol{C}_{0}(S \cup\{\delta\})$.

Proof. Let us set

(7. 29) $\quad V_{t}^{0} f([x, p])=\int_{S \cup\{\delta\}} \chi_{0}(t,[x, p],[d y, q]) f([y, q]), \quad f \in C_{0}(S \cup\{\delta\}), t \geqq 0$.

Then it holds by the definition (7.21) that

$$
V_{t}^{0} f([x, p])=E_{x}\left[e^{-\int_{0}^{t}\left|k\left(X_{s}^{\prime}\right)\right| d s} f\left(\left[X_{t}^{\prime}, p\right]\right)\right], \quad[x, p] \in S,
$$

where $f([x, p])$ is considered as a function on $E$ for fixed $p$. Since $H_{t}$ is: strongly continuous on $\boldsymbol{C}_{0}(E)$ and $k(x)$ is bounded continuous on $E$, the right hand side of $(7.30)$ belongs to $C_{0}(E)$ as a function of $x \in E$. Hence 
the semi-group $V_{t}^{0}$ is strongly continuous on $C_{0}(S \cup\{\delta\})$, because $V_{t}^{0} f(\delta)=f(\delta)$ for $t \geqq 0$.

On the other hand, we can see from Lemma 7. 1 and Lemma 7.3 that

$$
\begin{aligned}
\left\|V_{t}-V_{t}^{0}\right\| & =\sup _{[x, p] \in S} \int_{0}^{t} \int_{S \cup\{\delta\}} \Psi([x, p] ; d s,[d y, q]) \chi(t-s,[y, q], S \cup\{\delta\}) \\
& =\sup _{[x, p] \in S} \sum_{r=1}^{\infty} \int_{r}^{t} \int_{S \cup\{\delta\}} \Psi_{r}([x, p] ; d s,[d y, q]) \chi_{0}(t-s,[y, q], S \cup\{\delta\}) \\
& \leqq \sup _{[x, p] \in S} \sum_{r=1}^{\infty} \Psi_{r}([x, p] ; t, S \cup\{\delta\}) .
\end{aligned}
$$

Applying (7.27) to the right hand side of the above inequality, we have

$$
\left\|V_{t}-V_{t}^{0}\right\| \leqq \sum_{r=1}^{\infty} \frac{(\|k\| t)^{r}}{r !}=e^{\|k\| t}-1, \quad t \geqq 0 .
$$

Next we shall prove that $V_{t}$ maps $\left.\left.C_{0}(S \cup\} \delta\right\}\right)$ into itself. Set

$$
V_{t}^{(r)} f([x, p])=\int_{S \cup\{\delta\}} \chi_{r}(t,[x, p],[d y, q]) f([y, q]), f \in C_{0}(S \cup\{\delta\}), r \geqq 0, t \geqq 0 .
$$

As was proved already, $V_{t}^{(0)}=V_{t}^{0}$ maps $C_{0}(S \cup\{\delta\})$ into itself. Accordingly, we may use the mathematical induction. Assume that $V_{t}^{(r)}$ maps $\boldsymbol{C}_{0}(S \cup\{\delta\})$ into itself. Setting $k^{+}([x, p])=k^{+}(x)$ for $x \in E$ and $k^{+}(\delta)=0$, we can see from (7. 9) that

$$
\begin{aligned}
& V_{t}^{(r+1)} f([x, p]) \\
= & \int_{S \cup\{\delta\}} \int_{0}^{t} \int_{S \cup\{\delta\}} \Psi([x, p] ; d s,[d y, q]) \chi_{r}\left(t-s,[y, q],\left[d z, q^{\prime}\right]\right) f\left(\left[z, q^{\prime}\right]\right) \\
= & \int_{0}^{t} \int_{S \cup\{\delta\}} \Psi([x, p] ; d s,[d y, q]) V_{t-s}^{(r)} f([y, q]) \\
= & \int_{0}^{t} E_{x}\left[e^{-\int_{0}^{s}\left|k\left(X_{v}^{\prime}\right)\right| d v} k^{+}\left(\left[X_{s}^{\prime}, p+1\right]\right) V_{t-s}^{(r)} f\left(\left[X_{s}^{\prime}, p+1\right]\right) d s\right. \\
= & \int_{0}^{t} V_{s}^{0}\left(k^{+} V_{t-s}^{(r)} f\right)([x, p+1]) d s, \quad f \in C_{0}(S \cup\{\delta\}), \quad[x, p] \in S .
\end{aligned}
$$

Since $\left\|V_{t-s}^{(r)} f\right\| \leqq\|f\|$ and $V_{s}^{0}\left(k^{+} V_{t-s}^{(r)} f\right) \in C_{0}(S \cup\{\delta\})$, the above equation shows that $\left.\left(V_{t}^{(r+1)} f\right)\right|_{S} \in C_{0}(S)$. Also $\Psi(\delta ; \cdot, \cdot)=0$, and hence the above equation shows that $V_{t}^{(r+1)} f \in C_{0}(S \cup\{\delta\})$. Thus we can see that $V_{t}^{(r)} f \in C_{0}(S \cup\{\delta\})$ for any $f \in C_{0}(S \cup\{\delta\})$ and $r \geqq 1$.

Now the function 


$$
V_{t} f([x, p])=\sum_{r=0}^{\infty} V_{t}^{(r)} f([x, p]), \quad f \in C_{0}(S \cup\{\delta\}), t \geqq 0,
$$

belongs to $\boldsymbol{C}_{0}(S \cup\{\delta\})$, because $V_{t}^{(r)} f \in \boldsymbol{C}_{0}(S \cup\{\delta\})$ and, by (7. 27),

$$
\left\|V_{t}^{(r)} f\right\| \leqq \frac{(\|k\| t)^{r}}{r !}\|f\|
$$

holds for any $r \geqq 1$. Hence the strong continuity of $V_{t}^{0}$ on $\boldsymbol{C}_{0}(S \cup\{\delta\})$, (7. 31) and (7.13) prove that $V_{t}$ is a strongly continuous semi-group on $\boldsymbol{C}_{0}(S \cup\{\delta\})$.

The non-negative property of $V_{t}$ follows from the definitions of $\chi$ and $\chi_{r}$ and the contractive property of $V_{t}$ follows from (7. 11). Q.E.D.

New let us consider Markov processes on $S \cup\{\delta\}$. Since $V_{t}^{0}$ and $V_{t}^{(r)}$ satisfy the conditions $(a)$ and $(b)$ in Lemma 7.2, there exist two strong Markov processes $Y=\left\{Y_{t}=\left[X_{t}, N_{t}\right], \zeta, \mathscr{B}_{t}, P_{[x, p]} ;[x, p] \in S \cup\{\delta\}\right\}$ and $Y^{0}=\left\{Y_{t}^{0}=\left[X_{t}^{0}, N_{t}^{0}\right], \zeta^{0}, \mathscr{B}_{t}^{0}, P_{[x, p]}^{0} ;[x, p] \in S \cup\{\delta\}\right\}$ corresponding to the semigroups $V_{t}$ and $V_{t}^{0}$ respectively and a Markov time $\tau$ of $Y_{t}$ such that

$$
Y_{t}^{0}(w)=\left\{\begin{array}{cl}
Y_{t}(w), & \text { if } t<\tau \\
\delta, & \text { if } t \geqq \tau .
\end{array}\right.
$$

Also, we may assume that the sample paths of $Y_{t}$ are right continuous and quasi left continuous and, by Lemma 7.1 and Lemma 7.4, $Y_{t}$ is a conservative Markov process. Let us set

$$
\sigma_{r}(w)=\inf \left\{t>0 ; N_{t}(w)=N_{0}(w)+r\right\}, \quad r \geqq 0 .
$$

Then we have

Theorem 7.2. Let $\chi_{r}$ and $\Psi_{r}$ be measures given in (7.2). Let also $Y=\left\{Y_{t}=\left[X_{t}, N_{t}\right], \mathscr{B}_{t}, P_{[x, p]} ;[x, p] \in S \cup\{\delta\}\right\}$ be the strong Markov process mentioned above and let $\sigma_{r}$ be the Markov time given in (7. 34). Then we have

$$
\begin{aligned}
& P_{[x, p]}\left(Y_{t} \in B, \sigma_{r} \leqq t<\sigma_{r+1}\right)=\chi_{r}(t,[x, p], B) \\
& P_{[x, p j}\left(Y_{\sigma_{r}} \in B, \sigma_{r} \in d t\right)=\Psi_{r}([x, p] ; d t, B), \\
& \quad[x, p] \in S \cup\{\delta\}, B \in \mathscr{B}(S \cup\{\sigma\}), r \geqq 0 .
\end{aligned}
$$

Proof. If $[x, p]=\delta$, then (7.35) and (7.36) hold evidently. So we shall 
prove them for $[x, p] \in S$. Since it follows from the definitions of $\chi_{r}$ and $\Psi$ that

$$
\chi_{r}(t,[x, p],[E, p])=0, \quad[x, p] \in S, r \geqq 1,
$$

we have

$$
\begin{aligned}
P_{[x, p]}\left(Y_{t} \in B, t<\sigma_{1}\right) & =P_{[x, p]}\left(Y_{t} \in B, N_{t}=p, t<\sigma_{1}\right) \\
& \leqq \chi_{0}(t,[x, p], B) \\
& =P_{[x, p]}\left(Y_{t} \in B, t<\tau\right), \quad B \in \mathscr{B}(S \cup\{\delta\}),
\end{aligned}
$$

and hence

$$
P_{[x, p]}\left(\sigma_{1} \leqq \tau\right)=1, \quad[x, p] \in S
$$

On the other hand, $\chi_{0}(t,[x, p], \cdot)$ vanishes on $S \cup\{\delta\}-E \times\{p\}$ for any fixed $t \geqq 0$. Hence we have

$$
\begin{gathered}
P_{[x, p]}\left(N_{t}=p, t<\tau\right)=P_{[x, p]}^{0}\left(N_{t}^{0}=p, t<\tau\right)=P_{[x, p]}(t<\tau), \\
{[x, p] \in S,}
\end{gathered}
$$

which means

$$
P_{[x, p]}\left(\tau \leqq \sigma_{1}\right)=1, \quad[x, p] \in S
$$

Combining (7. 37) with the above equation, we can see that

$$
P_{[x, p]}\left(\tau=\sigma_{1}\right)=1 \text {, }
$$

and accordingly

$$
P_{[x, p]}\left(\tau_{r}=\sigma_{r}\right)=1, \quad[x, p] \in S, r \geqq 0,
$$

where $\tau_{0}=0, \tau_{1}=\tau$ and $\tau_{r+1}=\tau_{r}+\theta \tau_{r} \tau$.

The theorem follows from Lemma 7. 2 immediately.

Let us now consider the function defined by

$$
\widehat{f \cdot \lambda}([x, p])=\left\{\begin{array}{cc}
\lambda^{p} f(x), & {[x, p] \in S,} \\
0, & {[x, p]=\delta,}
\end{array}\right.
$$

for any $f \in \boldsymbol{B}(E)$ and $\lambda \geqq 0$. Then it follows from (7.12) and (7. 32) that 


$$
V_{t}|\widehat{f \cdot \lambda}|([x, p])=\sum_{r=0}^{\infty} \int_{[E, p+r]} \chi_{r}(t,[x, p],[d y, p+r])|\widehat{f \cdot \lambda}|([y, p+r])
$$

$$
\begin{aligned}
& \leqq \lambda^{p}\|f\| \sum_{r=0}^{\infty} \frac{(\lambda\|k\| t)^{r}}{r !} \\
& =\lambda^{p}\|f\| e^{\lambda\|k\| t}<\infty .
\end{aligned}
$$

Theorem 7.3. Let $V_{t}^{0}, V_{t}$ and $\Psi$ be semi-groups and measure given in (7. 29), (7.28) and (7.22) respectively. Let also $Y=\left\{Y_{t}=\left[X_{t}, N_{t}\right], \mathscr{B}_{t}, P_{[x, p]}\right.$; $[x, p] \in S \cup\{\delta\}\}$ be a conservative strong Markov process corresponding to $V_{t}$. Then the function $u(t, x)=V_{t} \widehat{f \cdot \lambda}([x, 0])$ is a solution of the following integral equation

$$
\begin{gathered}
u(t, x)=V_{t}^{0} \widehat{f \cdot \lambda}+\lambda \int_{0}^{t} \int_{E} u(t-s, y) \Psi([x, 0] ; d s,[d y, 1]), \\
x \in E, \quad t \geqq 0, \lambda \geqq 0, \quad f \in C_{0}(E),
\end{gathered}
$$

with initial value $u(0+, x)=f(x)$.

Proof. By (7. 38), $V_{t} \widehat{f \cdot \lambda}([x, 0])$ is bounded on $[0, T] \times\{S \cup\{\delta\}\}$ for any given $T>0$. Then we have

$$
\begin{aligned}
V_{t} \widehat{f \cdot \lambda}([x, 0]) & =E_{[x, 0]}\left[\widehat{f \cdot \lambda}\left(Y_{t}\right) ; t<\sigma\right]+E_{[x, 0]}\left[V_{t-\sigma} \widehat{f \cdot \lambda}\left(Y_{\sigma}\right) ; \sigma \leqq t\right] \\
& =V_{t}^{0} \widehat{f \cdot \lambda}([x, 0])+\int_{0}^{t} \int_{E} \Psi([x, 0] ; d s,[d y, 1]) V_{t-s} \widehat{f \cdot \lambda([y, 1]) .}
\end{aligned}
$$

Since $V_{t} \widehat{f \cdot \lambda}([x, p])=\lambda^{p} V_{t} \widehat{f \cdot \lambda}([x, 0])$, we can see that $u(t, x)=V_{t} \widehat{f \cdot \lambda}([x, 0])$ satisfies (7.39). Moreover, $V_{t}^{0}$ is a strongly continuous semi-group on $\boldsymbol{C}_{0}(S \cup\{\delta\})$ and hence we have

$$
\lim _{(t, x) \rightarrow\left(0, x_{0}\right)} V_{t} \widehat{f \cdot \lambda}([x, 0])=\lim _{(t, x) \rightarrow\left(0, x_{0}\right)} V_{t}^{0} \widehat{f \cdot \lambda}([x, 0])=\widehat{f \cdot \lambda}\left(\left[x_{0}, 0\right]\right)=f\left(x_{0}\right) .
$$

Thus we have proved the theorem.

Q.E.D.

Corollary 7.1. Let $X^{\prime}$ be a standard Brownian motion on $R^{d}$. If $k(x)$ is a bounded continuous function on $R^{a}$, then $u(t, x)=V_{t} \widehat{f \cdot 2([x, 0])}$ is a solution of the following differential equation 


$$
\frac{\partial u(t, x)}{\partial t}=-\frac{1}{2} \Delta u(t, x)+k(x) u(t, x), \quad x \in R^{d}, t \geqq 0,
$$

with initial value $f \in \boldsymbol{C}\left(R^{d}\right)$.

Proof. By the definition of $\Psi$, we have

$$
\begin{gathered}
\Psi([x, 0] ; d t,[B, 1])=E_{x}\left[e^{-\int_{0}^{t}\left|k\left(X_{\diamond}^{\prime}\right)\right| d s} k^{+}\left(X_{t}^{\prime}\right) I_{B}\left(X_{t}^{\prime}\right)\right] d t, \\
x \in R^{d}, B \in \mathscr{B}\left(R^{d}\right),
\end{gathered}
$$

where $E_{x}$ denotes the integral by the probability measure of a standard Brownian motion $X_{t}^{\prime}$. Then it follows from Theorem 7.3 that

$$
\begin{aligned}
u(t, x) & =V_{t}^{0} \widehat{f \cdot 2}([x, 0])+2 \int_{0}^{t} \int_{E \times\{1\}} \Psi([x, 0] ; d(t-s),[d y, 1]) u(s, y) \\
& =u_{0}(t, x)+v(t, x)
\end{aligned}
$$

where

$$
u_{0}(t, x)=E_{x}\left[e^{-2 \int_{0}^{t}\left|k\left(X_{s}^{\prime}\right)\right| d s} f\left(X_{t}^{\prime}\right)\right]
$$

and

$$
v(t, x)=2 \int_{0}^{t} E_{x}\left[e^{-2 \int_{0}^{t-s}\left|k\left(X_{v}^{\prime}\right)\right| d v} k^{+}\left(X_{t-s}^{\prime}\right) u\left(s, X_{t-s}^{\prime}\right)\right] d s .
$$

On the other hand, by Kac's theorem, ${ }^{38)}$ we have

$$
\frac{\partial u_{0}(t, x)}{\partial t}=\frac{1}{2} \Delta u_{0}(t, x)-|k(x)| u_{0}(t, x),
$$

and

$$
\begin{gathered}
\frac{\partial v(t, x)}{\partial t}=2 k^{+}(x) u(t, x)+\int_{0}^{t} \Delta E_{x}\left[e^{-2 \int_{0}^{t-s}\left|k\left(X_{v}^{\prime}\right)\right| d v} k^{+}\left(X_{t-s}^{\prime}\right) u\left(s, X_{t=s}^{\prime}\right)\right] d s \\
-|k(x)| v(t, x) .
\end{gathered}
$$

Combining (7.42) with the above two equations, we have

$$
\begin{aligned}
\frac{\partial u(t, x)}{\partial t} & =\frac{1}{2} \Delta u_{0}(t, x)-|k(x)| u_{0}(t, x)+2 k^{+}(x) u(t, x)+\frac{1}{2} \Delta v(t, x)-|k(x)| v(t, x) \\
& =\frac{1}{2} \Delta u(t, x)+\left(2 k^{+}(x)-|k(x)|\right) u(t, x) \\
& =\frac{1}{2} \Delta u+k(x) u(t, x) .
\end{aligned}
$$

38) cf. Ito-McKean [7], pp. 54-55. 
Since $u(0+, x)=f(x)$, the above equation proves the corollary.

Q.E.D.

\section{$\S 8$. Construction of a signed branching Markov processes with} age (II).

According to Theorem 5. 1, a strong Markov process on $\tilde{\boldsymbol{S}}$ satisfying Condition 3 is a signed branching Markov process with age. We shall construct such a process in this section.

Let $E$ be a compact Hausdorff space satisfying the second axiom of countability, and consider $S^{(n)}, S^{n}, \hat{\boldsymbol{S}}$ and $\tilde{\boldsymbol{S}}$ defined in $\S 2$. We shall define the mapping $\gamma$ from $\bigcup_{n=0}^{\infty} S^{(n)}$ into $S$ by

$$
r\left(\left(x_{1}, p_{1}\right),\left(x_{2}, p_{2}\right), \cdots,\left(x_{n}, p_{n}\right)\right)=\left[\left[x_{1}, x_{2}, \cdots, x_{n}\right],\left[p_{1}, p_{2}, \cdots, p_{n}\right]\right] \in S^{n} \text {. }
$$

Let $\left\{\left(q_{n}^{+}(x), q_{n}^{-}(x)\right) ; n=0,1,2, \cdots\right\}$ be a system of pairs of non-negative continuous functions on $E$ such that

$$
k(x)=\sum_{n=0}^{\infty}\left\{q_{n}^{+}(x)+q_{n}^{-}(x)\right\}, \quad x \in E,
$$

is bounded continuous on $E$, and

$$
q_{n}^{+}(x) q_{n}^{-}(x)=0, \quad x \in E, n=0,1,2, \cdots .
$$

Further let $X_{t}^{\prime}$ be a conservative Feller process on $E, H_{t}$ be the strongly continuous semi-group on $\boldsymbol{C}(E)$ induced by $X_{t}^{\prime}$, and let $Y=\left\{Y_{t}=\left[X_{t}, N_{t}\right]\right.$, $\left.\mathscr{B}_{t}, P_{[x, p]} ;[x, p] \in S\right\}$, where $S=E \times N$, be the strong Markov process constructed in $\S 7$ from the system $\left\{k(x), X_{t}^{\prime}\right\}$. (Since $k(x)$ is non-negative, the extra point $\delta$ is not needed.) Then, by (i) of Lemma 7.2, we may assume that almost all sample paths of $Y_{t}$ are right continuous and, for any given Markov time $\tau>0$, they have their left limit $Y_{\tau-}$ at the time $\tau$.

Let us set

$$
k([x, p])=k(x), \quad[x, p] \in S,
$$

and

$$
\varphi_{t}(w)=\int_{0}^{t} k\left(Y_{s}(w)\right) d s .
$$

We shall denote the $\exp \left(-\varphi_{t}\right)$ sub-process of $Y_{t}$ by $Y^{0}=\left\{Y_{t}^{0}=\left[X_{t}^{0}, N_{t}^{0}\right], \eta\right.$, $\left.\mathscr{B}_{t}^{0}, P_{[x, p]}^{0} ;[x, p] \in S\right\}$. Let also $Y_{i, t}^{0}, i=1,2, \cdots, n$, be Markov processes 
such that their fundamental spaces are identical to the one for $Y_{t}^{0}$, each of them is stochastically equivalent to $Y_{t}^{0}$ and they are mutually independent to each other. Then the probability measure of the joint process $\left(Y_{1, t}^{0}, Y_{2, t}^{0}\right.$, $\left.\cdots, Y_{n, t}^{0}\right)$ starting from $\left(\left(x_{1}, p_{1}\right),\left(x_{2}, p_{2}\right), \cdots,\left(x_{n}, p_{n}\right)\right) \in S^{(n)}$ is given by the product measure $P_{\left[x_{1}, p_{2}\right]}^{0} \times P_{\left[x_{1}, p_{2}\right]}^{0} \times \cdots \times P_{\left[x_{n}, p_{n}\right]}^{0}$. Using this product measure, we shall define a measure $\chi_{0}(t,[x, p], \cdot)$ on $\mathscr{B}(\hat{\mathbf{S}})$ by

$$
\chi_{0}(t,[\boldsymbol{x}, \boldsymbol{p}],[B, \boldsymbol{q}])
$$

$$
= \begin{cases}P_{\left[x_{1}, p_{2}\right]}^{0} \times P_{\left[x_{1}, p_{2}\right]}^{0} \times \cdots \times P_{\left[x_{n}, p_{n}\right]}^{0}\left(\left(Y_{1, t}^{0}, Y_{2, t}^{0}, \cdots, Y_{n, t}^{0}\right) \in \gamma^{-1}([B, q])\right), \\ \\ 1 & \text { if }[x, p] \neq \Delta,[\partial, p], \\ 1 & \text { if }[x, p]=[\partial, p] \text { and }[\partial, p] \in[B, \boldsymbol{q}], \\ 0 & \text { if }[x, p]=\Delta \text { and } \Delta \in[B, \boldsymbol{q}], \\ & , \text { otherwise, }\end{cases}
$$

where $\boldsymbol{x}=\left[x_{1}, x_{2}, \cdots, x_{n}\right], \boldsymbol{p}=\left[p_{1}, p_{2}, \cdots, p_{n}\right]$ and $B \in \mathscr{B}(\hat{\boldsymbol{S}})$.

Let us next define a measure $\Psi([\boldsymbol{x}, \boldsymbol{p}, j]) ; \cdot, \cdot)$ on $\mathscr{B}([0, \infty) \times \widetilde{\boldsymbol{S}})$. Using a given system $\left\{\left(q_{n}^{+}(x), q_{n}^{-}(x)\right) ; n=0,1,2, \cdots\right\}$, we shall define $\pi\left([x, p, j],\left[B, \boldsymbol{q}, j^{\prime}\right]\right)$ by (4. 1). Then a measure $\Psi\left([x, p, j] ; d t,\left[B, \boldsymbol{q}, j^{\prime}\right]\right)$ on $\mathscr{B}([0, \infty) \times \widetilde{\boldsymbol{S}})$ is defined by

$$
\begin{aligned}
\Psi\left([x, p, j] ; d t,\left[B, \boldsymbol{q}, j^{\prime}\right]\right)= & E_{[x, p]}^{0}\left(\pi\left(\left[X_{\eta_{-}}^{\circ}, N_{\eta_{-}}^{\circ}, j\right],\left[B, \boldsymbol{q}, j^{\prime}\right]\right) ; \eta \in d t\right) \\
& {[x, p, j] \in S \times J,\left[B, \boldsymbol{q}, j^{\prime}\right] \in \mathscr{B}(\widetilde{\boldsymbol{S}}), }
\end{aligned}
$$

where $Y_{t}^{0}=\left[X_{t}^{0}, N_{t}^{0}\right]$ is the Markov process mentioned above, $E_{[x, p]}^{0}$ denotes the integral by the probability measure $P_{[x, p]}^{0}$ of $Y_{t}^{0}$ and $J=\{0,1,2,3\}$. Then we shall extend the parameter space of $\Psi$ to $\widetilde{\boldsymbol{S}}$ as follows:

$$
\Psi(\Delta ; d t, \widetilde{\mathbf{S}})=\Psi([\partial, p, j] ; d t, \widetilde{\mathbf{S}})=0, \quad[\partial, p] \in S^{0},
$$

and for $[\boldsymbol{x}, \boldsymbol{p}]=\left[\left[x_{1}, x_{2}, \cdots, x_{n}\right],\left[p_{1}, p_{2}, \cdots, p_{n}\right]\right] \in S^{n}$

$$
\begin{aligned}
& \Psi\left([\boldsymbol{x}, \boldsymbol{p}, j] ; d t,\left[B, \boldsymbol{q}, j^{\prime}\right]\right) \\
= & \sum_{i=1}^{n} \int_{S} \Psi\left(\left[x_{i}, p_{i}, j\right] ; d t,\left[d \boldsymbol{y}, \boldsymbol{p}^{\prime}, j^{\prime}\right]\right) \chi_{0}\left(t,\left[\boldsymbol{x}_{i}^{\prime}, \boldsymbol{p}_{i}^{\prime}\right],\left[B_{y}, \boldsymbol{q}^{\prime}\right]\right),
\end{aligned}
$$

$$
[B, q] \in \mathscr{B}(\hat{\boldsymbol{S}}), j, j^{\prime} \in J,
$$

$\Psi([\boldsymbol{x}, \boldsymbol{p}, j] ; d t,\{\Delta\})=0$,

where $\chi_{0}$ is given in (8. 3), $\boldsymbol{x}_{i}^{\prime}=\left[x_{1}, \cdots, x_{i-1}, x_{i+1}, \cdots, x_{n}\right], \boldsymbol{p}_{i}^{\prime}=\left[p_{1}, \cdots\right.$, 
$\left.p_{i-1}, p_{i+1}, \cdots, p_{n}\right]$, and $\left[B_{y}, \boldsymbol{q}^{\prime}\right]$ denotes the Borel set $\left\{[\boldsymbol{z}, \boldsymbol{r}] \in \hat{\boldsymbol{S}} ; \boldsymbol{\gamma}\left(\boldsymbol{\gamma}^{-1}\left(\left[\boldsymbol{y}, \boldsymbol{p}^{\prime}\right]\right)\right.\right.$ $\left.\times \gamma^{-1}(z, r] \in[B, \boldsymbol{q}]\right\}$.

Now we shall define $\chi_{0}, \chi_{r}, \chi$ and $\Psi_{r}$ by

$$
\begin{aligned}
& \Psi_{1}\left([\boldsymbol{x}, \boldsymbol{p}, j] ; d t,\left[B, \boldsymbol{q}, j^{\prime}\right]\right)=\Psi\left([\boldsymbol{x}, \boldsymbol{p}, j] ; d t,\left[B, \boldsymbol{q}, j^{\prime}\right]\right) \\
& \left.\Psi_{r+1}\left([\boldsymbol{x}, \boldsymbol{p}, j] ; d t,\left[B, \boldsymbol{q}, j^{\prime}\right]\right)=\int_{0}^{t} \int_{\tilde{s}} \Psi_{r}([\boldsymbol{x}, \boldsymbol{p}, j]) ; d s,\left[d \boldsymbol{y}, \boldsymbol{p}^{\prime}, i\right]\right) \Psi\left(\left[\boldsymbol{y}, \boldsymbol{p}^{\prime}, i\right] ;\right. \\
& \left.d(t-s),\left[B, \boldsymbol{q}, j^{\prime}\right]\right), \\
& \Psi_{r}\left([\boldsymbol{x}, \boldsymbol{p}, j] ; t,\left[B, \boldsymbol{q}, j^{\prime}\right]\right)=\int_{0}^{t} \Psi_{r}\left([\boldsymbol{x}, \boldsymbol{p}, j] ; d s,\left[B, \boldsymbol{q}, j^{\prime}\right]\right), \\
& \text { (8. 7) } \quad \chi_{0}\left(t,[\boldsymbol{x}, \boldsymbol{p}, j],\left[B, \boldsymbol{q}, j^{\prime}\right]\right)=\boldsymbol{\delta}_{j, j^{\prime}} \chi_{0}(t,[\boldsymbol{x}, \boldsymbol{p}],[B, \boldsymbol{q}]) \text {, } \\
& \chi_{r}\left(t,[\boldsymbol{x}, \boldsymbol{p}, j],\left[B, \boldsymbol{q}, j^{\prime}\right]\right)=\int_{0}^{t} \int_{\tilde{S}} \Psi_{r}\left([\boldsymbol{x}, \boldsymbol{p}, j] ; d s,\left[d \boldsymbol{y}, \boldsymbol{p}^{\prime}, i\right]\right) \\
& \chi_{0}\left(t-s,\left[\boldsymbol{y}, \boldsymbol{p}^{\prime}, i\right],\left[B, \boldsymbol{q}, j^{\prime}\right]\right), \\
& \chi\left(t,[\boldsymbol{x}, \boldsymbol{p}, j],\left[B, \boldsymbol{q}, j^{\prime}\right]\right)=\sum_{r=0}^{\infty} \chi_{r}\left(t,[\boldsymbol{x}, \boldsymbol{p}, j],\left[B, \boldsymbol{q}, j^{\prime}\right]\right), \\
& {[\boldsymbol{x}, \boldsymbol{p}, j] \in \widetilde{\boldsymbol{S}},\left[B, \boldsymbol{q}, j^{\prime}\right] \in \mathscr{B}(\widetilde{\boldsymbol{S}}), r \geqq 1, t \geqq 0 .}
\end{aligned}
$$

Then we have

Lemma 8. 1. $\chi_{0}$ and $\Psi$ mentioned above satisfy the Moyal's $\chi_{0} \Psi$-condition, i.e. it holds that for any $[\boldsymbol{x}, \boldsymbol{p}, j] \in \widetilde{\boldsymbol{S}},\left[B, \boldsymbol{q}, j^{\prime}\right] \in \mathscr{B}(\widetilde{\boldsymbol{S}})$ and $t, s \geqq 0$

$$
\begin{array}{r}
\chi_{0}\left(t+s,[\boldsymbol{x}, \boldsymbol{p}, j],\left[B, \boldsymbol{q}, j^{\prime}\right]\right)=\int_{\tilde{s}} \chi_{0}\left(t,[\boldsymbol{x}, \boldsymbol{p}, j],\left[d \boldsymbol{y}, \boldsymbol{p}^{\prime}, i\right]\right) \\
\cdot \chi_{0}\left(s,\left[\boldsymbol{y}, \boldsymbol{p}^{\prime}, i\right],\left[B, \boldsymbol{q}, j^{\prime}\right]\right),
\end{array}
$$

$$
\Psi([\boldsymbol{x}, \boldsymbol{p}, j] ; \infty, \tilde{\mathbf{S}})=1-\lim _{t \rightarrow \infty} \chi_{0}(t,[\boldsymbol{x}, \boldsymbol{p}, j], \tilde{\mathbf{S}}),
$$

$$
\begin{aligned}
& \Psi\left([\boldsymbol{x}, \boldsymbol{p}, j] ; t+s,\left[B, \boldsymbol{q}, j^{\prime}\right]\right)=\Psi\left([\boldsymbol{x}, \boldsymbol{p}, j] ; t,\left[B, \boldsymbol{q}, j^{\prime}\right]\right) \\
& \quad+\int_{\tilde{s}} \chi_{0}\left(t,[\boldsymbol{x}, \boldsymbol{p}, j],\left[d \boldsymbol{y}, \boldsymbol{p}^{\prime}, i\right]\right) \Psi\left(\left[\boldsymbol{y}, \boldsymbol{p}^{\prime}, i\right] ; s,\left[B, \boldsymbol{q}, j^{\prime}\right]\right),
\end{aligned}
$$

$\Psi([\boldsymbol{x}, \boldsymbol{p}, j] ; t, \widetilde{\boldsymbol{S}})$ is continuous in $t$.

Proof. Since (8. 8) is evident from the definition of $\chi_{0}$ and also (8. 9)(8. 11) are evident when $[\boldsymbol{x}, \boldsymbol{p}, j]=\Delta$ or $[\boldsymbol{x}, \boldsymbol{p}, j]=[\partial, p, j]$, we shall prove (8. 9)-(8. 11) for $[\boldsymbol{x}, \boldsymbol{p}, j] \in \widetilde{\boldsymbol{S}}-\left(S^{0} \times J\right) \cup\{\Delta\}$.

Let $Y_{i, t}^{0}, i=1,2, \cdots, n$, be Markov processes and let $P_{\left[x_{1}, p_{1}\right]}^{0} \times P_{\left[x_{2}, p_{2}\right]}^{0} \times$ $\cdots \times P_{\left[x_{n}, p_{n}\right]}^{0}$ be the probability measure used in (8.3). Then it follows from (8.6) that 
$\Psi([\boldsymbol{x}, \boldsymbol{p}, j] ; d t, \widetilde{\mathbf{S}})$

$$
\begin{aligned}
& =\sum_{i=1}^{n} \int_{\tilde{S}} \Psi\left(\left[x_{i}, p_{i}, j\right] ; d t,\left[d \boldsymbol{y}, \boldsymbol{q}, j^{\prime}\right]\right) \chi_{0}\left(t,\left[\boldsymbol{x}_{i}^{\prime}, \boldsymbol{p}_{i}^{\prime}, 0\right], \tilde{\boldsymbol{S}}\right) \\
& =-d_{t} P_{\left[x_{1}, p_{1}\right]}^{0} \times \cdots \times P_{\left[x_{n}, p_{n}\right]}^{0}\left(\left(Y_{1, t}^{0}, \cdots, Y_{n, t}^{0}\right) \in \gamma^{-1} \hat{\boldsymbol{S}}\right), \\
& \quad[\boldsymbol{x}, \boldsymbol{p}]=\left[x_{1}, x_{2}, \cdots, x_{n}\right],\left[p_{1}, p_{2}, \cdots, p_{n}\right] \in S^{n}, n \geqq 1 .
\end{aligned}
$$

Hence we have

$$
\begin{aligned}
\Psi([\boldsymbol{x}, \boldsymbol{p}, j] ; t, \tilde{\mathbf{S}}) & =-\int_{0}^{t} d_{s} P_{\left[x_{1}, p_{1}\right]}^{0} \times \cdots \times P_{\left[x_{n}, p_{n}\right]}^{0}\left(\left(Y_{1, t}^{0}, \cdots, Y_{n, t}^{0}\right) \in \gamma^{-1} \hat{\mathbf{S}}\right) \\
& =1-\chi_{0}(t,[\boldsymbol{x}, \boldsymbol{p}, j], \widetilde{\mathbf{S}}), \quad[\boldsymbol{x}, \boldsymbol{p}, j] \in \widetilde{\boldsymbol{S}}, t \geqq 0,
\end{aligned}
$$

which proves (8. 9).

We shall next show (8. 10). Considering the process $\left(Y_{1, t}^{0}, \cdots, Y_{n, t}^{0}\right)$ mentioned above, we have for $[\boldsymbol{x}, \boldsymbol{p}]=\left[\left[x_{1}, x_{2}, \cdots, x_{n}\right],\left[p_{1}, p_{2}, \cdots, p_{n}\right]\right]$

$$
\begin{aligned}
& \Psi\left([\boldsymbol{x}, \boldsymbol{p}, j] ; t+s,\left[B, \boldsymbol{q}, j^{\prime}\right]\right) \\
= & \Psi\left([\boldsymbol{x}, \boldsymbol{p}, j] ; t,\left[B, q, j^{\prime}\right]\right)+\int_{t}^{t+s} \Psi\left([\boldsymbol{x}, \boldsymbol{p}, j] ; d v,\left[B, \boldsymbol{q}, j^{\prime}\right]\right) \\
= & \Psi\left([\boldsymbol{x}, \boldsymbol{p}, j] ; t,\left[B, \boldsymbol{q}, j^{\prime}\right]\right) \\
& \quad+\int_{t}^{t+s} \sum_{i=1}^{n} \int_{\hat{\boldsymbol{s}}} \Psi\left(\left[x_{i}, p_{i}, j\right] ; d v,\left[d \boldsymbol{y}, \boldsymbol{p}^{\prime}, j^{\prime}\right]\right) \chi_{0}\left(v,\left[\boldsymbol{x}_{i}^{\prime}, \boldsymbol{p}_{i}^{\prime}, j^{\prime}\right],\left[B_{y}, \boldsymbol{q}^{\prime}, j^{\prime}\right]\right) \\
= & \Psi\left([\boldsymbol{x}, \boldsymbol{p}, j] ; t,\left[B, \boldsymbol{q}, j^{\prime}\right]\right)+\int_{0}^{s} \sum_{i=1}^{n} \int_{\hat{S}} E_{\left[x_{i}, p_{i}\right]}^{0}\left[\Psi\left(\left[X_{i, t}^{0}, N_{i, t}^{0}, j\right] ; d v,\left[d \boldsymbol{y}, \boldsymbol{p}^{\prime}, j^{\prime}\right]\right)\right] \\
& \cdot E_{\left[\boldsymbol{x}_{i}{ }^{\prime}, \boldsymbol{p}_{i}^{\prime}\right]}^{0}\left[\chi_{0}\left(v,\left[X_{i, t}^{\prime}, N_{i, t}^{\prime}, j^{\prime}\right],\left[B_{y}, \boldsymbol{q}^{\prime}, j^{\prime}\right]\right)\right],
\end{aligned}
$$

where $E_{[\boldsymbol{x}, \boldsymbol{p}]}^{0}$ denotes the integral by $P_{\left[x_{1}, p_{1}\right]}^{0} \times \cdots \times P_{\left[x_{n}, p_{n}\right]}^{0}$ for $[\boldsymbol{x}, \boldsymbol{p}]=\left[\left[x_{1}, x_{2}\right.\right.$, $\left.\left.\cdots, x_{n}\right],\left[p_{1}, p_{2}, \cdots, p_{n}\right]\right]$ and $\left[X_{i, t}^{\prime 0}, N_{i, t}^{\prime, 0}\right]$ denotes $\left[Y_{1, t}^{0}, \cdots, Y_{i-1, t}^{0}, Y_{i+1, t}^{0}\right.$, $\left.\cdots, Y_{n, t}^{0}\right]$. Then the right hand side of the above equation is equal to

$$
\begin{aligned}
& \Psi\left([\boldsymbol{x}, \boldsymbol{p}, j] ; t,\left[B, \boldsymbol{q}, j^{\prime}\right]\right)+\int_{0}^{s} E_{[\boldsymbol{x}, \boldsymbol{p}]}^{0}\left[\sum_{i=1}^{n} \int_{\hat{s}} \Psi\left(\left[X_{i, t}^{0}, N_{i, t}^{0}, j\right] ; d v,\left[d \boldsymbol{y}, \boldsymbol{p}^{\prime}, j^{\prime}\right]\right)\right. \\
& \left.\cdot \chi_{0}\left(v,\left[X_{i, t}^{\prime}, N_{i, t}^{\prime}, 0\right],\left[B_{y}, \boldsymbol{q}^{\prime}, 0\right]\right)\right] \\
= & \Psi\left([\boldsymbol{x}, \boldsymbol{p}, j] ; t,\left[B, \boldsymbol{q}, j^{\prime}\right]\right) \\
& +\int_{0}^{s} E_{[\boldsymbol{x}, \boldsymbol{p}]}^{0}\left[\Psi\left(\left[\left[X_{1, t}^{0}, X_{2, t}^{0}, \cdots, X_{n, t}^{0}\right],\left[N_{1, t}^{0}, N_{2, t}^{0}, \cdots, N_{n, t}^{0}\right], j\right] ; d v,\left[B, \boldsymbol{q}, j^{\prime}\right]\right)\right] \\
= & \Psi\left([\boldsymbol{x}, \boldsymbol{p}, j] ; t,\left[B, \boldsymbol{q}, j^{\prime}\right]\right)
\end{aligned}
$$




$$
+\int_{\tilde{s}} \chi_{0}\left(t,[\boldsymbol{x}, \boldsymbol{p}, j],\left[d \boldsymbol{y}, \boldsymbol{p}^{\prime}, i\right]\right) \Psi\left([\boldsymbol{y}, \boldsymbol{p}, i] ; s,\left[B, \boldsymbol{q}, j^{\prime}\right]\right)
$$

So we have (8. 10) for any $[\boldsymbol{x}, \boldsymbol{p}, j] \in \tilde{\boldsymbol{S}}$.

Now $k(x)$ is bounded continuous and hence $\Psi([x, p, j] ; \cdot, \widetilde{\mathbf{S}})$ is absolutely continuous with respect to the Lebesgue measure on $[0, \infty)$. Then (8.6) proves (8. 11) for any $[\boldsymbol{x}, \boldsymbol{p}, j] \in \tilde{\boldsymbol{S}}$.

Now we shall consider the linear operators $U_{t}^{(r)}$ and $U_{t}$ on $\boldsymbol{B}(\widetilde{\boldsymbol{S}})$ defined by

$$
U_{t}^{(r)} h([\boldsymbol{x}, \boldsymbol{p}, j])=\int_{\tilde{s}} \chi_{r}\left(t,[\boldsymbol{x}, \boldsymbol{p}, j],\left[d \boldsymbol{y}, \boldsymbol{q}, j^{\prime}\right]\right) h\left(\left[\boldsymbol{y}, \boldsymbol{q}, j^{\prime}\right]\right),
$$

$$
\left.U_{t} h([\boldsymbol{x}, \boldsymbol{p}, j])=\int_{\tilde{s}} \chi(t,[\boldsymbol{x}, \boldsymbol{p}, j]),\left[d \boldsymbol{y}, \boldsymbol{q}, j^{\prime}\right]\right) h\left(\left[\boldsymbol{y}, \boldsymbol{q}, j^{\prime}\right]\right),
$$

$$
[\boldsymbol{x}, \boldsymbol{p}, j] \in \widetilde{\boldsymbol{S}} .
$$

Further set

$$
\widehat{f \cdot \lambda}([x, p, j])=\widetilde{f \cdot \lambda}([x, p, 0]), \quad f \in \boldsymbol{B}(E),[\boldsymbol{x}, \boldsymbol{p}, j] \in \widetilde{\boldsymbol{S}} .
$$

Then we have

Lemma 8. 2. Let $U_{t}^{(0)}$ be the operator defined above. Then $U_{t}^{(0)}$ is strongly continuous on $\boldsymbol{C}_{0}(\widetilde{\boldsymbol{S}})$.

Proof. By Theorem 7. 1, the semi-group $V_{t}$ corresponding to the process $Y_{t}=\left[X_{t}, N_{t}\right]$ on $S$ is strongly continuous on $C_{0}(S)$, while $k([x, p])=k(x)(\geqq 0)$ is bounded and continuous on $S$. Hence $U_{t}^{(0)}$ is strongly continuous on $\boldsymbol{C}_{0}(S)$.

Now suppose $h \in \boldsymbol{C}_{0}(\widetilde{\boldsymbol{S}})$ and set

$$
\left.h\right|_{S^{n} \times\{j\}}([\boldsymbol{x}, \boldsymbol{p}, j])=\left\{\begin{array}{cl}
h([\boldsymbol{x}, \boldsymbol{p}, j]), & \text { if }[\boldsymbol{x}, \boldsymbol{p}] \in S^{n}, n \geqq 0, \\
0, & \text { otherwise. }
\end{array}\right.
$$

Then we have

$$
U_{t}^{(0)} h([\boldsymbol{x}, \boldsymbol{p}, j])=U_{t}^{0}\left(\left.h\right|_{S^{n} \times J}\right)([\boldsymbol{x}, \boldsymbol{p}, j]), \quad[\boldsymbol{x}, \boldsymbol{p}, j] \in S^{n} \times\{j\}, n \geqq 0 .
$$

On the other hand, the linear hull of $\left\{\widehat{f \cdot \lambda} ; f \in C^{*}(E), 0 \leqq \lambda<1\right\}$ is dense in $\boldsymbol{C}_{0}(\hat{\boldsymbol{S}})$ and $\left.U_{t}^{(0)} \widehat{f \cdot \lambda}\right|_{S^{n} \times\{j\}} \in \boldsymbol{C}_{0}\left(S^{n} \times\{j\}\right)$ which follows from $\left.U_{t}^{\cos } \widehat{f \cdot \lambda}\right|_{S^{n} \times\{j\}}$ $=\widehat{\left.\left.\left(U_{t}^{(0)} \widehat{f} \cdot \lambda\right)\right|_{E} \cdot \lambda\right|_{S^{n} x\{j\}}}$ and $\left.U_{t}^{(0)} \widehat{f \cdot \lambda}\right|_{E} \in C_{0}(S)$. So, for any $\varepsilon>0, n \geqq 0$ and $j \in J$, we can find constants $\alpha_{i}, f_{i} \in C^{*}(E)$ and $0 \leqq \lambda_{i}<1, i=1,2, \cdots, i_{n}$, which may depend on $\varepsilon, n$ and $j$, such that 


$$
\| h-\sum_{i=1}^{i_{n}} \alpha_{i}\left(\widehat{\left.f_{i} \cdot \lambda_{i}\right)} \|_{S^{n} \times\{j\}}<\varepsilon^{39)}\right. \text {. }
$$

Then (8. 13) and the contraction property of $U_{t}^{(0)}$ imply that $U_{t}^{(0)} h \in \boldsymbol{C}_{0}(\widetilde{\boldsymbol{S}})$.

Next we shall show the strong continuity of $U_{t}^{(0)}$ on $\boldsymbol{C}_{0}(\widetilde{\boldsymbol{S}})$. As was stated already, $U_{t}^{(0)}$ is strongly continuous on $C_{0}(S)$. Hence we have

$$
\begin{array}{r}
\left\|U_{t}^{(0)} \widehat{f \cdot \lambda}-\widehat{f \cdot \lambda}\right\|_{S \times J} \rightarrow 0 \text { as } t \rightarrow 0, \\
f \in C^{*}(E), 0 \leqq \lambda<1 .
\end{array}
$$

Then, for any $[\boldsymbol{x}, \boldsymbol{p}]=\left[\left[x_{1}, x_{2}, \cdots, x_{n}\right],\left[p_{1}, p_{2}, \cdots, p_{n}\right]\right]$ and $j \in J$, it follows from the definitions of $U_{t}^{(0)}$ and $\chi_{0}$ that

$$
\begin{aligned}
& \left|U_{t}^{(0)} \widehat{f \cdot \lambda}([\boldsymbol{x}, \boldsymbol{p}, j])-\widehat{f \cdot \lambda}([\boldsymbol{x}, \boldsymbol{p}, j])\right| \\
& \leqq\left|\prod_{i=1}^{n} U_{t}^{(0)} \widehat{f \cdot \lambda}\left(\left[x_{i}, p_{i}, 0\right]\right)-\prod_{i=1}^{n} \widehat{f \cdot \lambda}\left(\left[x_{i}, p_{i}, 0\right]\right)\right| \\
& =\mid \sum_{r=1}^{n}\left\{U_{t}^{(0)} \widehat{f \cdot \lambda}\left(\left[x_{r}, p_{r}, 0\right]\right)-\widehat{f \cdot \lambda}\left(\left[x_{r}, p_{r}, 0\right]\right)\right\} \\
& \cdot \prod_{i=1}^{r-1} \widehat{f \cdot \lambda}\left(\left[x_{i}, p_{i}, 0\right)\right] \prod_{i=r+1}^{n} U_{t}^{(0)} \widehat{f \cdot \lambda}\left(\left[x_{i}, p_{i}, 0\right]\right) \mid \\
& \leqq C(f, \lambda)\left\|U_{t}^{(0)} \widehat{f \cdot \lambda}-\widehat{f \cdot \lambda}\right\|_{S \times J}, \quad f \in C^{*}(E), 0 \leqq \lambda<1 \text {, }
\end{aligned}
$$

where $C(f, \lambda)$ is a constant defined by

$$
C(f, \lambda)=\sup \left\{n\|\widehat{f \cdot \lambda}\|_{S \times J}^{n-1} ; n=1,2,3, \cdots\right\}
$$

Combining (8. 15) and (8. 16), we have

$$
\lim _{t \rightarrow 0}\left\|U_{t}^{(0)} \widehat{f \cdot \lambda}-\widehat{f \cdot \lambda}\right\|_{\tilde{\boldsymbol{S}}}=0, \quad f \in C^{*}(E), 0 \leqq \lambda<1
$$

because $U_{t}^{(0)} \widehat{f \cdot \lambda}([\partial, p, j])=\widehat{f \cdot \lambda}([\partial, p, j])$ and $U_{t}^{(0)} \widehat{f \cdot \lambda}(\Delta)=\widehat{f \cdot \lambda}(\Delta)=0$. Then it follows from (8.14) and (8.17) that for any fixed $n \geqq 0$ and $j \in J$

$$
\lim _{t \rightarrow 0}\left\|\left.U_{t}^{(0)} h\right|_{S^{n} \times\{j\}}-\left.h\right|_{S^{n} \times\{j\}}\right\|_{\tilde{\boldsymbol{S}}}=0, \quad h \in \boldsymbol{C}_{0}(\widetilde{\boldsymbol{S}}) .
$$

39) For any function $f$ on a topological space $\mathscr{X}$, we denote in the sequel $\sup \left\{|f(x)| ; x \in A \subset \mathscr{X}^{\rho}\right\}$ by $\|f\|_{A}$. 
On the other hand, $h$ and $U_{t}^{(0)} h$ are elements of $\boldsymbol{C}_{0}(\widetilde{\boldsymbol{S}})$. Hence there exists an $n_{0}$ such that

$$
\left\|U_{t}^{(0)} h-h\right\|_{\tilde{S}} \leqq \max _{n \leqq n_{0}, j \in J}\left\|\left.U_{t}^{(0)} h\right|_{S^{n} \times\{j\}}-h_{S^{n} \times\{j\}}\right\|_{\tilde{S}}
$$

Therefore we can see from (8. 18) that $U_{t}^{(0)}$ is strongly continuous on $\boldsymbol{C}_{0}(\tilde{\mathbf{S}})$.

$$
\text { Q.E.D. }
$$

Now let $Y_{i, t}=\left[X_{i, t}, N_{i, t}\right], \quad i=1,2, \cdots, n$, be Markov processes on $S$ such that their fundamental spaces are identical to the one of $Y_{t}$, each of them is stochastically equivalent to $Y_{t}=\left[X_{t}, N_{t}\right]$ and mutually independent to each other. Then the probability measure of the joint process $\left(Y_{1, t}, Y_{2, t}\right.$, $\left.\cdots, Y_{n, t}\right)$ is given by the product measure $P_{\left[x_{1}, p_{1}\right]} \times P_{\left[x_{2}, p_{2}\right]} \times \cdots \times P_{\left[x_{n}, p_{n}\right]}$. The integral by the probability measure $P_{\left[x_{1}, p_{1}\right]} \times P_{\left[x_{2}, p_{2}\right]} \times \cdots \times P_{\left[x_{n}, p_{n}\right]}$ is denoted by $E_{(\boldsymbol{x}, \boldsymbol{p})}$ when $\boldsymbol{x}=\left[x_{1}, x_{2}, \cdots, x_{n}\right]$ and $\boldsymbol{p}=\left[p_{1}, p_{2}, \cdots, p_{n}\right]$. Then the process $Y_{i, t}^{0}$ mentioned already can be considered as the $\exp \left(-\varphi_{t}\right)$ subprocess of $Y_{i, t}$.

We shall next define the set $D_{n}$ by

$$
D_{n}=\left\{\boldsymbol{x} ; \boldsymbol{x}=[x, x, \cdots, x] \in E^{n}\right\}
$$

Then, by (8. 4), $\Psi([x, p, 0] ; d s,[\cdot, \boldsymbol{q}, \cdot])$ vanishes outside of $\left(\bigcup_{n=0}^{\infty} D_{n}\right) \times\{1,3\}$. Hence it follows from (8.6) and (8.7) that for $[\boldsymbol{x}, \boldsymbol{p}]=\left[\left[x_{1}, x_{2}, \cdots, x_{n}\right]\right.$, $\left.\left[p_{1}, p_{2}, \cdots, p_{n}\right]\right]$

$$
\begin{aligned}
& \Psi([\boldsymbol{x}, \boldsymbol{p}, 0] ; d t,[B, \boldsymbol{q}, 1]) \\
&= \sum_{i=1}^{n} \int_{\hat{S}} \Psi\left(\left[x_{i}, p_{i}, 0\right] ; d t,\left[d \boldsymbol{y}, \boldsymbol{p}^{\prime}, 1\right]\right) \chi_{0}\left(t,\left[\boldsymbol{x}_{i}^{\prime}, \boldsymbol{p}_{i}^{\prime}, 0\right],\left[B_{y}, \boldsymbol{q}^{\prime}, 0\right]\right) \\
&= \sum_{i=1}^{n} \int_{\hat{S}} E_{\left[x_{i}, p_{i}\right]}^{0}\left[\pi\left(\left[Y_{i, \eta-}^{0}, N_{i, \eta-}^{0}, 0\right],\left[d \boldsymbol{y}, \boldsymbol{p}^{\prime}, 1\right]\right) ; \eta \in d t\right] \\
& \cdot P_{\left[x_{1}, p_{1}\right]}^{0} \times \cdots \times P_{\left[x_{i-1}, p_{i-1}\right]}^{0} \times P_{\left[x_{i+1}, p_{i+1}\right]}^{0} \times \cdots \times P_{\left[x_{n}, p_{n}\right]}^{0} \\
&\left(( Y _ { 1 , t } ^ { 0 } , \cdots , Y _ { i - 1 , t } ^ { 0 } , Y _ { i + 1 , t } ^ { 0 } , \cdots , Y _ { n , t } ^ { 0 } ) \in \gamma ^ { - 1 } \left(\left[B_{y}, q^{\prime}\right]\right.\right. \\
&=\sum_{i=1}^{n} \int_{\hat{S}} E_{\left(x_{i}, p_{i}\right)}\left[e^{-\int_{0}^{t} k\left(Y_{i, s}\right) d s} k\left(Y_{i, t}\right) \pi\left(\left[X_{i, t}, N_{i, t}, 0\right],\left[d \boldsymbol{y}, \boldsymbol{p}^{\prime}, 1\right]\right)\right] \\
& \quad \cdot E_{\left(\boldsymbol{x}_{\left.i^{\prime}, \boldsymbol{p}_{i}\right)}\right)}\left[e^{-\sum_{r * i}^{\Sigma} \int_{0}^{t} k\left(Y_{\left.r_{r, s}\right)}\right) d s}\right. \\
&\left.\cdot I_{\gamma^{-1}\left(\left[B_{y}, \boldsymbol{q}^{\prime}\right]\right)}\left(Y_{1, t}, \cdots, Y_{i-1, t}, Y_{i+1, t}, \cdots, Y_{n, t}\right)\right] d t
\end{aligned}
$$




$$
\begin{aligned}
=\int_{\hat{S}} E_{(\boldsymbol{x}, \boldsymbol{p})}\left[e ^ { - \sum _ { i = 1 } ^ { n } \int _ { 0 } ^ { t } k ( Y _ { i , s } ) d s } \sum _ { i = 1 } ^ { n } \left\{\sum_{m=0}^{\infty} q_{m}^{+}\left(X_{i, t}\right) \boldsymbol{\delta}_{m}\left(Y_{i, t},\left[d \boldsymbol{y}, \boldsymbol{p}^{\prime}\right]\right)\right.\right. \\
\left.\left.\cdot I_{\gamma^{-1}\left(\left[B_{y}, \boldsymbol{q}^{\prime}\right]\right)}\left(Y_{1, t}, \cdots, Y_{i-1, t}, Y_{i+1, t}, \cdots, Y_{n, t}\right)\right\}\right] d t,
\end{aligned}
$$

where $\left[\boldsymbol{x}_{i}^{\prime}, \boldsymbol{p}_{i}^{\prime}\right]=\left[\left[x_{1}, \cdots, x_{i-1}, x_{i+1}, \cdots, x_{n}\right],\left[p_{1}, \cdots, p_{i-1}, p_{i+1}, \cdots, p_{n}\right]\right]$ and $\left[B_{y}, \boldsymbol{q}^{\prime}\right]=\left\{[\boldsymbol{z}, \boldsymbol{r}] \in \hat{\boldsymbol{S}} ; \boldsymbol{\gamma}\left(\boldsymbol{\gamma}^{-1}\left(\left[\boldsymbol{y}, \boldsymbol{p}^{\prime}\right]\right) \times \boldsymbol{\gamma}^{-1}([\boldsymbol{z}, \boldsymbol{r}]) \in[B, \boldsymbol{q}]\right\}\right.$. Similarly, we have

$$
\begin{aligned}
& \Psi([\boldsymbol{x}, \boldsymbol{p}, 0]; d t,[B, \boldsymbol{q}, 3]) \\
&=\int_{\hat{S}} E_{(\boldsymbol{x}, \boldsymbol{p})}\left[e ^ { - \sum _ { i = 1 } ^ { n } \int _ { 0 } ^ { t } k ( Y _ { i , s } ) d s } \cdot \sum _ { i = 1 } ^ { n } \left\{\sum_{m=0}^{\infty} q_{m}^{-}\left(X_{i, t}\right) \delta_{m}\left(Y_{i, t},\left[d \boldsymbol{y}, \boldsymbol{p}^{\prime}\right]\right)\right.\right. \\
&\left.\left.\cdot I_{\gamma^{-1}\left(\left[B_{v}, \boldsymbol{q}^{\prime}\right]\right)}\left(Y_{1, t}, \cdots, Y_{i-1, t}, Y_{i+1, t}, \cdots, Y_{n, t}\right)\right\}\right] d t .
\end{aligned}
$$

Then we have

Lemma 8. 3. Let $U_{t}^{(r)}$ be the operator on $\boldsymbol{B}(\widetilde{\boldsymbol{S}})$ given in (8. 12). Then $U_{t}^{(r)}$ maps $\boldsymbol{C}_{0}(\widetilde{\boldsymbol{S}})$ into itself. Moreover it holds that

$$
\lim _{t \rightarrow 0}\left\|U_{t}^{(r)} h\right\|=0, \quad h \in C_{0}(\widetilde{\boldsymbol{S}}), r \geqq 1 .
$$

Proof. We shall first prove that $U_{t}^{(r)} h \in \boldsymbol{C}_{0}(\widetilde{\boldsymbol{S}})$ for any $h \in \boldsymbol{C}_{0}(\widetilde{\boldsymbol{S}}) . \quad$ By Lemma 8. 2, $U_{t}^{(0)}$ is strongly continuous on $\boldsymbol{C}_{0}(\widetilde{\boldsymbol{S}})$. So it suffices to prove that for $h \in \boldsymbol{C}_{0}(\widetilde{\boldsymbol{S}}) U_{t}^{(r+1)} h$ is continuous in $(t,[\boldsymbol{x}, \boldsymbol{p}, j])$ as a function on $[0, \infty) \times \widetilde{\boldsymbol{S}}$ and $U_{t}^{(r+1)} h \in \boldsymbol{C}_{0}(\widetilde{\boldsymbol{S}})$ for any fixed $t \geqq 0$ under the assumption that $U_{t}^{(r)} h$ satisfies the same properties.

Now, by (8. 7) and (8. 12), we have

$$
U_{t}^{(r+1)} h([x, p, 0])=\int_{0}^{t} \int_{\tilde{s}} \Psi([\boldsymbol{x}, \boldsymbol{p}, 0] ; d s,[d \boldsymbol{y}, \boldsymbol{q}, j]) U_{t-s}^{(r)} h([\boldsymbol{y}, \boldsymbol{q}, j]) .
$$

Applying (8.19) and (8.20) to the right hand side of the above equation, we can see that for $[\boldsymbol{x}, \boldsymbol{p}]=\left[\left[x_{1}, x_{2}, \cdots, x_{n}\right],\left[p_{1}, p_{2}, \cdots, p_{n}\right]\right]$

$$
\begin{aligned}
& U_{t}^{(r+1)} h([\boldsymbol{x}, \boldsymbol{p}, 0]) \\
= & \int_{0}^{t} E_{(\boldsymbol{x}, \boldsymbol{p})}\left[e^{-\sum_{i=1}^{n} \int_{0}^{s} k\left(\left[X_{i, v}, N_{i, v}\right]\right) d v}\right. \\
\cdot \sum_{i=1}^{n} \sum_{m=0}^{\infty}\left\{q _ { m } ^ { + } ( X _ { i , s } ) U _ { t - s } ^ { ( r ) } h \left(\left[X_{1, s}, \cdots, X_{i-1, s}\right.\right.\right. & \underbrace{X_{i, s}, \cdots, X_{i, s}}_{m}, X_{i+1, s}, \cdots, X_{n, s}], \\
& {\left.\left.[N_{1, s}, \cdots, N_{i-1, s}, N_{i, s}, \underbrace{0,0, \cdots, 0}_{m-1}, N_{i+1, s}, \cdots, N_{n, s}], 1\right]\right) }
\end{aligned}
$$




$$
\begin{gathered}
+q_{m}^{-}\left(X_{i, s}\right) U_{t-s}^{(r)} h\left(\left[[X_{1, s}, \cdots, X_{i-1, s}, \underbrace{X_{i, s}, \cdots, X_{i, s}}_{m}, X_{i+1, s}, \cdots, X_{n, s}],\right.\right. \\
\left.\left.\left.\left.[N_{1, s}, \cdots, N_{i-1, s}, N_{i, s}, \underbrace{0,0, \cdots, 0}_{m-1}, N_{i+1, s}, \cdots, N_{n, s}], 3\right]\right)\right\}\right] d s .
\end{gathered}
$$

To prove the right hand side of (8.22) is continuous in $(t,[\boldsymbol{x}, \boldsymbol{p}])$ and also belongs to $\boldsymbol{C}_{0}(\hat{\boldsymbol{S}})$ for any fixed $t \geqq 0$, we consider the following function

$$
\begin{gathered}
g(s ;[\boldsymbol{x}, \boldsymbol{p}]) \\
=\sum_{i=1}^{n} \sum_{m=0}^{\infty}\left\{q _ { m } ^ { + } ( x _ { i } ) U _ { s } ^ { ( r ) } h \left(\left[[x_{1}, \cdots, x_{i-1}, \underbrace{x_{i}, \cdots, x_{i}}_{m}, x_{i+1}, \cdots, x_{n}],\right.\right.\right. \\
\left.\left.\quad[p_{1}, \cdots, p_{i-1}, p_{i}, \underbrace{0, \cdots, 0}_{m-1}, p_{i+1}, \cdots, p_{n}], 1\right]\right) \\
+q_{m}^{-}\left(x_{i}\right) U_{s}^{(r)} h\left(\left[[x_{1}, \cdots, x_{i-1}, \underbrace{x_{i}, \cdots, x_{i}, x_{i+1}}_{m}, \cdots, x_{n}],\right.\right. \\
\left.\left.\left.[p_{1}, \cdots, p_{i-1}, p_{i}, \underbrace{0, \cdots, 0}_{m-1}, p_{i+1}, \cdots, p_{n}], 3\right]\right)\right\},
\end{gathered}
$$

where $[\boldsymbol{x}, \boldsymbol{p}]=\left[\left[x_{1}, x_{2}, \cdots, x_{n}\right],\left[p_{1}, p_{2}, \cdots, p_{n}\right]\right]$. By the assumption of induction, $U_{s}^{(r)} h$ is bounded and continuous on $[0, T] \times \widetilde{\boldsymbol{S}}$ for any given $T>0$ and belongs to $\boldsymbol{C}_{0}(\widetilde{\boldsymbol{S}})$ for any fixed $t \geqq 0$. On the other hand, $\sum_{m=0}^{\infty}\left\{q_{m}^{+}(x)+q_{m}^{-}(x)\right\}$ converges to $k(x)$ uniformly on the compact space $E$ because $\left\{q_{m}^{+}(x)+q_{m}^{-}(x)\right\} \geqq 0$ and $k(x)$ is continuous. Hence the right hand side of (8.23) is the sum of uniformly convergent series of continuous functions, and accordingly $g(s ;[\boldsymbol{x}, \boldsymbol{p}])$ is continuous in $(s,[\boldsymbol{x}, \boldsymbol{p}])$. Moreover we can see that $g(s ;[\boldsymbol{x}, \boldsymbol{p}])$ belongs to $\boldsymbol{C}_{0}(\hat{\boldsymbol{S}})$ for any fixed $t \geqq 0$.

Now we have from (8.22) and (8.23)

$$
\begin{aligned}
& U_{t}^{(r+1)} h([\boldsymbol{x}, \boldsymbol{p}, 0]) \\
= & \int_{0}^{t} E_{(\boldsymbol{x}, \boldsymbol{p})}\left[e^{-\sum_{i=1}^{n} \int_{0}^{s} k\left(\left[X_{i, v}, Y_{i, v}\right]\right) d v}\right. \\
& \left.\cdot g\left(t-s ;\left[\left[X_{1, s}, X_{2, s}, \cdots, X_{n, s}\right],\left[N_{1, s}, N_{2, s}, \cdots, N_{n, s}\right]\right]\right)\right] d s .
\end{aligned}
$$

Since the semi-group $V_{t}$ corresponding to $Y_{i, t}=\left[X_{i, t}, N_{i, t}\right]$ is strongly continuous on $\boldsymbol{C}_{0}(S)$ and $g(s ;[\boldsymbol{x}, \boldsymbol{p}])$ is bounded and continuous on $[0, T] \times \hat{\boldsymbol{S}}$, the integrand of the right hand side of the above equation is also continuous on $[0, T] \times \hat{S}$. Hence $U_{t}^{(r+1)} h([\boldsymbol{x}, \boldsymbol{p}, 0])$ is continuous in $(t,[\boldsymbol{x}, \boldsymbol{p}])$ and belongs 
to $\boldsymbol{C}_{0}(\hat{\boldsymbol{S}})$ for any fixed $t \geqq 0$ because $g(t,[\boldsymbol{x}, \boldsymbol{p}]) \in \boldsymbol{C}_{0}(\hat{\boldsymbol{S}})$ for fixed $t \geqq 0$. Similarly, we can see that $U_{t}^{(r+1)} h([\boldsymbol{x}, \boldsymbol{p}, j]), j \in J$, are continuous in $(t,[\boldsymbol{x}, \boldsymbol{p}])$ and belong to $\boldsymbol{C}_{0}(\hat{\boldsymbol{S}})$ for any fixed $t \geqq 0$. Hence $U_{t}^{(r+1)} h$ is continuous in $(t,[\boldsymbol{x}, \boldsymbol{p}, j])$ and belongs to $\boldsymbol{C}_{0}(\widetilde{\boldsymbol{S}})$ for any fixed $t \geqq 0$.

Next we shall prove (8. 21). Let $r \geqq 1$. Since $U_{t}^{(r)} h$ is continuous on a compact set $[0, T] \times \widetilde{\boldsymbol{S}}$ as a function of $(t,[\boldsymbol{x}, \boldsymbol{p}, j])$ and vanishes on $[0, T] \times\{\Delta\}$, it holds that for any $\varepsilon>0$, there exists an $n_{0}$ such that

$$
\sup _{n \geq n_{0}}\left\|U_{t}^{(r)} h\right\|_{S^{n} \times J}<\varepsilon, \quad 0 \leqq t \leqq T .
$$

On the other hand, it follows from (8. 22) that

$$
\begin{aligned}
& \left|U_{t}^{(r)} h([\boldsymbol{x}, \boldsymbol{p}, j])\right| \\
\leqq & \int_{0}^{t} E_{(\boldsymbol{x}, \boldsymbol{p})}\left[e^{-\sum_{i=1}^{n} \int_{0}^{t} k\left(\left[X_{i, v}, N_{i, v}\right]\right) d v} \sum_{i=1}^{n} \sum_{m=0}^{\infty}\left(q_{m}^{+}\left(X_{i, s}\right)+q_{m}^{-}\left(X_{i, s}\right)\right) \sup _{0 \leqq s \leqq t}\left\|U_{t-s}^{(r-1)} h\right\| d s\right.
\end{aligned}
$$

$$
\begin{aligned}
& =\sup _{0 \leqq s \leqq t}\left\|U_{s}^{(r-1)} h\right\| \int_{0}^{t} E_{(\boldsymbol{x}, \boldsymbol{p})}\left[e^{-\sum_{i=1}^{n} \int_{0}^{s} k\left(\left[X_{i, v}, N_{i, v}\right]\right) d v} \sum_{i=1}^{n} k\left(\left[X_{i, s}, N_{i, s}\right]\right)\right] d s \\
& =\sup _{0 \leqq s \leqq t}\left\|U_{s}^{(r-1)} h\right\|\left(1-e^{-n\|\boldsymbol{k}\| t}\right), \quad[\boldsymbol{x}, \boldsymbol{p}, j] \in \tilde{\boldsymbol{S}}, t \geqq 0 .
\end{aligned}
$$

Since $U_{s}^{(r-1)} h$ is bounded on $[0, T] \times \widetilde{\boldsymbol{S}}$, there exists a constant $M$ such that

$$
\sup _{0 \leqq s \leqq T}\left\|U_{s}^{(r-1)} h\right\| \leqq M<\infty .
$$

Then (8. 24) and (8. 25) show us the following inequality.

$$
\left\|U_{t}^{(r)} h\right\| \leqq M\left(1-e^{-n_{0}\|k\| t}\right)+\varepsilon, \quad 0 \leqq t \leqq T .
$$

This proves (8. 21) because $\varepsilon$ is arbitrary.

We are now in a position to state the following

Theorem 8. 1. Let $\left\{\left(q_{n}^{+}(x), q_{n}^{-}(x)\right) ; n=0,1,2, \cdots\right\}$ be a given system of pairs of non-negative continuous functions on $E$ such that

$$
k(x)=\sum_{n=0}^{\infty}\left(q_{n}^{+}(x)+q_{n}^{-}(x)\right), \quad x \in E,
$$

is bounded continuous on $E$, and

$$
q_{n}^{+}(x) q_{n}^{-}(x)=0, \quad n=0,1,2, \cdots
$$

Then there exists a signed branching Markov process with age $Z=\left\{Z_{t}=\left[X_{t}, N_{t}, J_{t}\right]\right.$, $\left.\zeta, \mathscr{B}_{t}, P_{[\boldsymbol{x}, \boldsymbol{p}, j]} ;[\boldsymbol{x}, \boldsymbol{p}, j] \in \widetilde{\mathbf{S}}\right\}$ on $\tilde{\mathbf{S}}$ satisfying Condition 3 for a given $\left\{\left(q_{n}^{+}(x), q_{n}^{-}(x)\right)\right.$; $n=0,1,2, \cdots\}$. 
Proof. According to Lemma 7. 2 and Lemma 8.2-8.3, there exists a right continuous strong Markov process $Z=\left\{Z_{t}=\left[X_{t}, N_{t}, J_{t}\right], \zeta, \mathscr{B}_{t}, P_{[\boldsymbol{x}, \boldsymbol{p}, j]}\right.$; $[\boldsymbol{x}, \boldsymbol{p}, j] \in \tilde{\boldsymbol{S}}\}$ on $\tilde{\boldsymbol{S}}$ corresponding to the semi-group $U_{t}$ given in (8.12) and a $\mathscr{B}_{t}$-Markov time $\eta$ such that

$$
P_{[\boldsymbol{x}, \boldsymbol{p}, j]}\left(Z_{t} \in B, \eta_{r} \leqq t<\eta_{r+1}\right)=\chi_{r}(t,[\boldsymbol{x}, \boldsymbol{p}, j], B),
$$

$$
\begin{aligned}
P_{[\boldsymbol{x}, \boldsymbol{p}, j]}\left(Z_{\eta_{r}} \in B, \eta_{r} \in d t\right) & =\Psi_{r}([\boldsymbol{x}, \boldsymbol{p}, j] ; d t, B), \\
& {[\boldsymbol{x}, \boldsymbol{p}, j] \in \widetilde{\boldsymbol{S}}, B \in \mathscr{B}(\widetilde{\boldsymbol{S}}), r \geqq 0, t \geqq 0, }
\end{aligned}
$$

where

$$
\eta_{0}=0, \eta_{1}=\eta, \eta_{r+1}=\eta_{r}+\theta_{\eta_{r}} \eta, r \geqq 1
$$

Let us set

$$
\tilde{\eta}(w)=\inf \left\{t>0 ; J_{t}(w) \neq J_{0}(w) \text { or } \sup _{s \leqq t}\left|N_{s}(w)\right|=\infty\right\} .
$$

Since we can see from (8. 4), (8.6) and (8.26) that

$$
P_{[\boldsymbol{x}, \boldsymbol{p}, j]}\left(J_{\eta}=J_{0} \text { or } \sup _{s \leqq \eta}\left|N_{s}(w)\right|=\infty\right)=0, \quad[\boldsymbol{x}, \boldsymbol{p}, j] \in \widetilde{\boldsymbol{S}},
$$

we have

$$
P_{[\boldsymbol{x}, \boldsymbol{p}, j]}(\tilde{\eta}>\eta)=0, \quad[\boldsymbol{x}, \boldsymbol{p}, j] \in \widetilde{\boldsymbol{S}}
$$

On the other hand, $\chi_{0}(t,[\boldsymbol{x}, \boldsymbol{p}, j], \cdot)$ vanishes outside of $\hat{\boldsymbol{S}} \times\{j\}$. Hence we have

$$
P_{[\boldsymbol{x}, \boldsymbol{p}, j]}\left(J_{t} \neq J_{0}, t<\eta\right)=0,
$$

which means

$$
P_{[\boldsymbol{x}, \boldsymbol{p}, j]}\left(J_{s}=J_{0} \text { for any } s \leqq t<\eta\right)=P_{[\boldsymbol{x}, \boldsymbol{p}, j]}(t<\eta),
$$

because $J_{t}$ is right continuous. So we have

$$
P_{[\boldsymbol{x}, \boldsymbol{p}, j]}(\tilde{\eta} \neq \eta)=0, \quad[\boldsymbol{x}, \boldsymbol{p}, j] \in \tilde{\boldsymbol{S}},
$$

which means that we may regard $\eta_{r}$ is the $r$ th branching time of $Z_{t}$.

Now we shall check the conditions in Condition 3 for our process.

(a) (i) of Condition 1 follows from (8.4), (8.26) and the definition of $Y_{t}^{0}$ which was used to construct $\chi_{0}$. 
(b) Let $X_{t}^{\prime}$ be the conservative Feller process on $E$ and let $Y_{t}=\left[X_{t}, N_{t}\right]$ be the strong Markov process on $S$ which are mentioned in the first part of this section. Then it follows from the way of constructions of $\chi_{0}$ and $\Psi$, that

$$
\begin{aligned}
& P_{[x, 0, j]}\left(\left[X_{\eta-}, N_{\eta]}\right] \in[B, n], \eta \in d t\right) \\
= & E_{[x, 0]}\left[e^{-\int_{0}^{t} k\left(\left[X_{s}, N_{s}\right]\right) d s} k\left(\left[X_{t}, N_{t}\right]\right) I_{B \times\{n\}}\left(\left[X_{t}, N_{t}\right]\right)\right] d t \\
= & E_{x}\left[e^{-2 \int_{0}^{t} k\left(X_{s}^{\prime}\right) d s} k\left(X_{t}^{\prime}\right) I_{B}\left(X_{t}^{\prime}\right) \frac{\left(\int_{0}^{t} k\left(X_{s}^{\prime}\right) d s\right)^{n}}{n !}\right] d t, x \in E, j \in J, B \in \mathscr{B}(E) .
\end{aligned}
$$

where $E_{[x, p]}$ and $E_{x}$ denote the integrals by the probability measures of $Y_{t}$ and $X_{t}^{\prime}$ respectively, and $k([x, p])=k(x)$. So (3. 3) holds. Similarly, (3. 4) holds. Moreover, by the definitions of $\chi_{0}, \Psi$ and $\Psi_{r}$, we have

$$
\begin{aligned}
& \chi_{0}(t,[\boldsymbol{x}, \boldsymbol{p}, j],[B, \boldsymbol{q}, J])=\chi_{0}\left(t,\left[\boldsymbol{x}, \boldsymbol{p}, j^{\prime}\right],[B, \boldsymbol{q}, J]\right), \\
& \Psi([\boldsymbol{x}, \boldsymbol{p}, j] ; d t,[B, \boldsymbol{q}, J])=\Psi\left(\left[\boldsymbol{x}, \boldsymbol{p}, j^{\prime}\right] ; d t,[B, \boldsymbol{q}, J]\right),
\end{aligned}
$$

and hence

$$
\begin{array}{r}
\Psi_{r}([\boldsymbol{x}, \boldsymbol{p}, j] ; d t,[B, \boldsymbol{q}, J])=\Psi_{r}\left(\left[\boldsymbol{x}, \boldsymbol{p}, j^{\prime}\right] ; d t,[B, \boldsymbol{q}, J]\right), \\
{[\boldsymbol{x}, \boldsymbol{p}] \in \hat{\boldsymbol{S}}, j, j^{\prime} \in J,[B, \boldsymbol{q}] \in \mathscr{B}(\hat{\boldsymbol{S}}) .}
\end{array}
$$

So we have

$$
\begin{aligned}
\chi(t,[\boldsymbol{x}, \boldsymbol{p}, j],[B, \boldsymbol{q}, J]) & =\chi\left(t,\left[\boldsymbol{x}, \boldsymbol{p}, j^{\prime}\right],[B, \boldsymbol{q}, J]\right), \\
{[\boldsymbol{x}, \boldsymbol{p}] } & \in \hat{\boldsymbol{S}}, j, j^{\prime} \in J,[B, \boldsymbol{q}] \in \mathscr{B}(\hat{\boldsymbol{S}}) .
\end{aligned}
$$

Thus our process satisfies (i) of Condition 2.

(c) (4. 2) follows from (8. 26) and (8.4). (4. 3) follows also from Theorem 7.2 and (7.22).

Combining $(a)-(c)$, we can see that our process satisfies (i) of Condition 3.

(d) (ii) of Condition 3 follows from (8.3), (8.7) and the definition of s $\sim$ ".

(e) (iii) of Condition 3 follows from (8.6).

(f) (iv) of Condition 3 follows from (8. 3), (8. 5) and (8. 7).

(g) By the definition of $\chi$ and (8.26), we have 


$$
\begin{aligned}
P_{[\boldsymbol{x}, \boldsymbol{p}, j]}\left(Z_{t} \in \widetilde{\boldsymbol{S}}\right) & =\sum_{r=0}^{\infty} U_{t}^{(r)} I_{\tilde{\boldsymbol{S}}}([\boldsymbol{x}, \boldsymbol{p}, j]) \\
& =\sum_{r=0}^{\infty} P_{[\boldsymbol{x}, \boldsymbol{p}, j]}\left(\eta_{r} \leqq t<\eta_{r+1}\right) \\
& =P_{[\boldsymbol{x}, \boldsymbol{p}, j]}\left(t<\eta_{\infty}\right),
\end{aligned}
$$

$[\boldsymbol{x}, p, j] \in \tilde{\boldsymbol{S}}$.

Therefore we may consider that

$$
P_{[x, p, j]}\left(\eta_{\infty}<\zeta\right)=0, \quad[\boldsymbol{x}, \boldsymbol{p}, j] \in \widetilde{\boldsymbol{S}} .
$$

If we consider a new process $\tilde{Z}_{t}$ defined by

$$
\tilde{Z}_{t}(w)=\left\{\begin{array}{cl}
Z_{t}(w), & \text { if } t<\eta_{\infty}(w) \wedge \zeta(w), \\
\Delta, & \text { if } t \geqq \eta_{\infty}(w) \wedge \zeta(w),
\end{array}\right.
$$

and Borel field $\tilde{\mathscr{B}}_{t}$ induced naturally from $\mathscr{B}_{t}$, then $\tilde{Z}_{t}$ satisfies (v) of Condition 3.

We shall denote $\tilde{Z}_{t}$ by $Z_{t}$ again. Then $(a)-(g)$ implies that our process $Z_{t}$ satisfies Condition 3. Moreover, by Theorem 5. 1, $Z_{t}$ is a signed branching Markov process with age on $\widetilde{\boldsymbol{S}}$.

Q.E.D.

Corollary 8.1. Let $\left\{\left(q_{n}^{+}(x), q_{n}^{-}(x)\right) ; n=0,1,2, \cdots\right\}$ be a given system of pairs of non-negative continuous functions on $E$ such that $k(x)=\sum_{n=0}^{\infty}\left(q_{n}^{+}(x)+q_{n}^{-}(s)\right)$ is bounded continuous on $E$ and $q_{n}^{+}(x) q_{n}^{-}(x)=0, n=0,1,2, \cdots$ Then there exists a signed branching Markov process $Z=\left\{Z_{t}, \zeta, \mathscr{B}_{t}, P_{[\boldsymbol{x}, \boldsymbol{p}, j]} ;[\boldsymbol{x}, \boldsymbol{q}, j] \in \widetilde{\boldsymbol{s}}\right\}$ on $\tilde{\mathbf{s}}$ satisfying Condition 2 for a given $\left\{\left(q_{1 b}^{+}(x), q_{n}^{-}(x)\right) ; n=0,1,2, \cdots\right\}$.

Corollary 8.2. Let $\left\{q_{n}(x) ; n=0,2,3, \cdots\right\}$ be a given system of nonnegative continuous functions on $E$ such that $k(x)=\sum_{n \neq 1} q_{n}(x)$ is bounded continuous on $E$. Then there exists a branching Markov process with age $Y=\left\{Y_{t}=\left[X_{t}, N_{t}\right]\right.$, $\left.\zeta, \mathscr{B}_{t}, P_{[\boldsymbol{x}, \boldsymbol{p}]} ;[\boldsymbol{x}, \boldsymbol{p}] \in \hat{\boldsymbol{S}}\right\}$ on $\hat{\boldsymbol{S}}$ satisfying Condition 1 for a given $\left\{q_{n}(x) ; n=0,2\right.$, $3, \cdots\}$.

Proof. Let us consider in Theorem 8. 1 the special case where $q_{n}^{-}(x)=0$, $n=0,1,2, \cdots, \quad$ and $\quad q_{1}^{+}(x)=0 . \quad$ Let $Z=\left\{Z_{t}=\left[X_{t}, N_{t}, J_{t}\right], \zeta, \mathscr{B}_{t}, P_{[\boldsymbol{x}, \boldsymbol{p}, j]}\right.$; $[\boldsymbol{x}, \boldsymbol{p}, j] \in \widetilde{\boldsymbol{S}}\}$ be the process obtained in Theorem 8.1 for the present case. Setting 


$$
\xi_{t}(w)= \begin{cases}n, & \text { if }\left[X_{t}(w), N_{t}(w)\right] \in S^{n}, n \geqq 0, \\ \infty, & \text { if }\left[X_{t}(w), N_{t}(w)\right]=\Delta,\end{cases}
$$

and

$$
\tau(w)=\inf \left\{t>0 ; \xi_{t}(w) \neq \xi_{0}(w) \text { or } \sup _{s \leqq t}\left|N_{s}(w)\right|=\infty\right\}
$$

we have

$$
P_{[x, p, 0]}(\tau \neq \eta)=0, \quad[x, p] \in \hat{S} \text {. }
$$

Also it follows from the definition of $\Psi$ that

$$
P_{[\boldsymbol{x}, \boldsymbol{p}, 0]}\left(J_{t}=2 \text { or } 3\right)=0, \quad[\boldsymbol{x}, \boldsymbol{p}] \in \hat{\boldsymbol{S}}, t \geqq 0,
$$

and hence we have

$$
\left.P_{[\boldsymbol{x}, \boldsymbol{p}, 0]} \widetilde{(f \cdot \lambda}\left(Z_{t}\right)=\widehat{f \cdot \lambda}\left(Z_{t}\right), t<\zeta\right)=P_{[\boldsymbol{x}, \boldsymbol{p}, 0]}(t<\zeta), \quad[\boldsymbol{x}, \boldsymbol{p}] \in \hat{\boldsymbol{S}} .
$$

So, if we disregard $J_{t}$ in $Z_{t}=\left[X_{t}, N_{t}, J_{t}\right]$ and define $P_{[x, p]}$ by

$$
P_{[\boldsymbol{x}, \boldsymbol{p}]}\left(\left[X_{t}, N_{t}\right] \in[B, q]\right)=P_{[\boldsymbol{x}, \boldsymbol{p}, 0]}\left(\left[Z_{t} \in[B, q, J]\right),\right.
$$

then the process $Y=\left\{Y_{t}=\left[X_{t}, N_{t}\right], \zeta, \mathscr{B}_{t}, P_{[\boldsymbol{x}, \boldsymbol{p}]} ;[\boldsymbol{x}, \boldsymbol{p}] \in \hat{\boldsymbol{S}}\right\}$ satisfies Condition 1 and

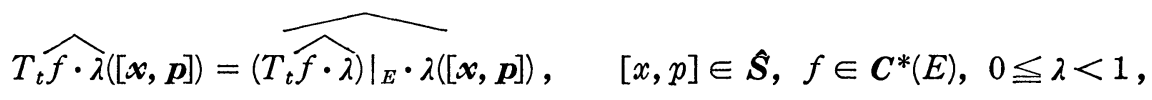

where $T_{t}$ denotes the semi-group on $\boldsymbol{B}(\hat{\boldsymbol{S}})$ induced by $Y$. Q.E.D.

\section{REFERENCES}

[1] E.B. Dynkin: Markov processes. Springer 1965.

[2] —: Markov processes and semi-group of operators. Th. of Prob. \& its appl. Vol. 1 (1956), pp. 22-33.

[ 3 ] G.A. Hunt: Markov processes and potentials II. III. Jour. Math., Vol. 2 (1958), pp. 151-213.

[4] N. Ikeda, M. Nagasawa and S. Watanabe: Foundation of branching Markov processes. Seminar on Probability, Vol. 23 (1966), (in Japanese).

[5] — On branching Markov processes. Proc. Japan Acad. Vol. 41 (1965), pp. 816-821.

[6] — Fundamental equations of branching Markov processes. Proc. Japan Acad. Vol. 42 (1966), pp. 252-257.

[7] —: Branching Markov Processes. (to appear). 
[ 8 ] K. Ito and H.P. McKean, Jr.: Diffusion processes and their sample paths. Springer, 1965.

[9] A. Kolmogoroff, I. Petrovsky and N. Piscounoff: Etude de l'équation de la diffusion avec croissance de la quantité de matière et son application à un problème biologique. Bull. l'Univ. Moscou, Vol. 1, Fasc. 6, pp. 1-25.

[10] J.E. Moyal: Discontinuous Markov processes. Acta. Math., Vol. 98 (1957), pp. 221264.

[11] : The general theory of stochastic population processes. Acta. Math., Vol. 108 (1962), pp. 1-32.

[12] —: Multiplicative population processes. Jour. Appl. Prob. Vol. 1 (1964), pp. 267-283.

[13] M. Nagasawa: Construction of branching Markov processes with age and sign (to appear).

[14] M. Nagasawa and T. Sirao: Probabilistic treatment of blowing up of solutions for a non-linear integral equation (to appear).

[15] H.J. Ryser: Combinatorial Mathematics. John Wiely Sons, 1963.

[16] T. Sirao: A probabilistic treatment of semi-linear parabolic equations. Proc. Japan Acad. Vol. 42 (1966), pp. 885-890.

[17] —: Remarks on the Moyal's construction of Markov processes. (to appear).

[18] A.V. Skorohod: Branching diffusion processes. Th. of Prob. \& its appl. Vol. 9 (1964), pp. 492-497.

Nagoya University 